José Francisco Roca Reyes

\title{
Flow of a capsule suspended in a Newtonian liquid through a constricted channel and capillary
}

Tese de Doutorado

Thesis presented to the Programa de Pós-graduação em Engenharia Mecânica of PUC-Rio in partial fulfillment of the requirements for the degree of Doutor em Engenharia Mecânica.

Advisor : Prof. Márcio da Silveira Carvalho Co-advisor: Prof. Ivan Fabio Mota de Menezes 

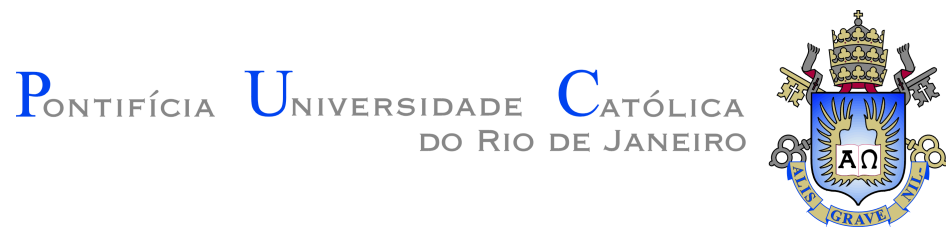

José Francisco Roca Reyes

\title{
Flow of a capsule suspended in a Newtonian liquid through a constricted channel and \\ capillary
}

Thesis presented to the Programa de Pós-graduação em Engenharia Mecânica of PUC-Rio in partial fulfillment of the requirements for the degree of Doutor em Engenharia Mecânica. Approved by the Examination Committee:

\author{
Prof. Márcio da Silveira Carvalho \\ Advisor \\ Departamento de Engenharia Mecânica - PUC-Rio \\ Prof. Ivan Fabio Mota de Menezes \\ Co-advisor \\ Departamento de Engenharia Mecânica - PUC-Rio \\ Prof. Aristeu da Silveira Neto \\ Faculdade de Engenharia Mecânica - UFU \\ Prof. Rafael Menezes de Oliveira \\ Departamento de Engenharia Mecânica - PUC-Rio
}

Prof. Roney Leon Thompson

Departamento de Engenharia Mecânica - COPPE/UFRJ

Prof. Taygoara Felamingo de Oliveira Departamento de Engenharia Mecânica - UnB 
All rights reserved.

\section{José Francisco Roca Reyes}

Majored in mechatronics in the Department of Mechanical Engineering at the Universidad Nacional de Ingeniería (Perú). In 2011, obtained his M.Sc. in the Department of Mechanical Engineering at Pontifícia Universidade Católica do Rio de Janeiro (Brasil).

Bibliographic data

Roca Reyes, José Francisco

Flow of a capsule suspended in a Newtonian liquid through a constricted channel and capillary / José Francisco Roca Reyes; advisor: Márcio da Silveira Carvalho; co-advisor: Ivan Fabio Mota de Menezes. - 2021.

103 f. : il. color. ; $30 \mathrm{~cm}$

Tese (doutorado) - Pontifícia Universidade Católica do Rio de Janeiro, Departamento de Engenharia Mecânica, 2021.

Inclui bibliografia

1. Engenharia Mecânica - Teses. 2. Cápsulas. 3. Interação fluido-estrutura. 4. Método de fronteira imersa. 5. Método de elementos finitos. I. Carvalho, Márcio da Silveira. II. Menezes, Ivan Fabio Mota de. III. Pontifícia Universidade Católica do Rio de Janeiro. Departamento de Engenharia Mecânica. IV. Título. 
To my parents, for their love and support all the way through this. 


\section{Acknowledgments}

First, I would like to thank my advisor Prof. Márcio Carvalho whose guidance and advice throughout these years helped me to finish this work. Certainly, without his support I would not have finished this thesis.

I would also like to express my gratitude to my co-advisor Prof. Ivan Menezes for his patience and caring in the development of this work, specially by helping me to improve the code implementation.

I must thank the rest of the examination committee for their insightful comments and suggestions which certainly enriched this work.

I would like to thank all my current and former colleagues at the Laboratory of Microhydrodynamics and Flow in Porous Media at PUC-Rio. Throughout these years, I was lucky to work with talented and hard-working people on numerical and experimental studies.

This study was financed in part by the Coordenação de Aperfeiçoamento de Pessoal de Nível Superior - Brasil (CAPES) - Finance Code 001 and was carried out in association with the project registered as Escoamento de dispersões complexas em meios porosos heterogêneos: análise na escala de poro e macroscópica (PUC-Rio/BG Brasil/Shell Brasil/ANP) - Complex Dispersion Flow Through Porous Media - funded by BG Brasil/Shell Brasil under the ANP R\&D levy as Compromisso de Investimentos com Pesquisa e Desenvolvimento.

Last but not least, I would like to thank my parents and my brother Juan Carlos for their encouragement, specially in these difficult pandemic times. My beloved parents, Rita and José, because they supported me emotionally all the way through this work. Finally, I would like to thank my partner Beatriz for her decisive support to finish this work, and of course my beloved little children, José Gabriel and María Eduarda, who patiently waited for me to conclude this work, thank you kids. 


\section{Abstract}

Roca Reyes, José Francisco; Carvalho, Márcio da Silveira (Advisor); Menezes, Ivan Fabio Mota de (Co-Advisor). Flow of a capsule suspended in a Newtonian liquid through a constricted channel and capillary. Rio de Janeiro, 2021. 103p. Tese de Doutorado - Departamento de Engenharia Mecânica, Pontifícia Universidade Católica do Rio de Janeiro.

The flow of capsules suspended in a liquid phase through small channels and capillaries poses a complex problem presented in different applications, from red blood cells on hemodynamics to flow in porous media. In applications of porous media, the understanding of microscale dynamics is fundamental to assess the macroscopic flow behavior. Constricted channels and capillaries can be used to model a pore throat connecting two adjacent pore bodies. The flow of a suspended capsule through such models was analyzed to evaluate the flow characteristics considering inertial effects (i.e. finite Reynolds numbers), including the maximum pressure difference required to push a capsule through the constriction as a function of capsule radius, initial membrane tension, membrane material, channel and capillary geometries, as well as flow conditions. In fact, in this study, the pressure response is fundamental in order to assess the capsule blocking mechanism. Inner and outer liquid phases were described by the Navier-Stokes equations and capsule membrane dynamics was modeled by a 1-D spring-like flexible structure. The fluid-structure interaction problem was solved using the finite element method coupled with the immersed boundary method. Results showed the mobility reduction of the continuous phase due to the presence of a capsule as it flows through the constriction. Such results can be used to design microcapsules to block preferential water flow paths in oil displacement process in porous media.

\section{Keywords}

Capsules; Fluid-structure interaction; Immersed boundary method; Finite element method. 


\section{Resumo}

Roca Reyes, José Francisco; Carvalho, Márcio da Silveira; Menezes, Ivan Fabio Mota de. Escoamento de cápsulas suspensas em um líquido Newtoniano através de um canal e capilar com constrição. Rio de Janeiro, 2021. 103p. Tese de Doutorado - Departamento de Engenharia Mecânica, Pontifícia Universidade Católica do Rio de Janeiro.

O escoamento de cápsulas suspensas em uma fase líquida através de canais e capilares micrométricos representa um problema complexo que ocorre em diferentes aplicações, de glóbulos vermelhos em hemodinâmica até escoamento em meios porosos. Em aplicações de meios porosos, a compreensão da dinâmica na microescala é fundamental para avaliar o comportamento macroscópico do escoamento. Canais e capilares com constrição podem ser usados para modelar uma garganta conectando dois poros adjacentes. O escoamento de uma cápsula suspensa através de tais modelos foi analisado para avaliar as características do escoamento considerando os efeitos inerciais (isto é, número de Reynolds finito), incluindo a máxima diferença de pressão necessária para empurrar uma cápsula através da constrição em função do raio da cápsula, a tensão inicial e o material da membrana, geometria do canal e do capilar, assim como as condições de escoamento. De fato, neste estudo, a resposta da pressão é fundamental para avaliar o efeito de bloqueio da cápsula. As fases líquidas internas e externas foram descritas pelas equações de Navier-Stokes, enquanto que a dinâmica da membrana da cápsula foi modelada por uma estrutura flexível 1-D tipo mola. O problema de interação fluido-estrutura foi resolvido usando o método de elementos finitos acoplado ao método de fronteira imersa. Os resultados mostraram a redução da mobilidade da fase contínua devido à presença da cápsula através da constrição. Tais resultados podem ser usados para projetar microcápsulas para bloquear caminhos preferenciais de fluxo da água no processo de deslocamento de óleo em meios porosos.

\section{Palavras-chave}

Cápsulas; Interação fluido-estrutura; Método de fronteira imersa; Método de elementos finitos. 


\section{Table of contents}

$\begin{array}{llr}1 & \text { Introduction } & 18\end{array}$

$\begin{array}{lll}1.1 & \text { Objectives } & 19\end{array}$

$\begin{array}{lll}1.2 & \text { Thesis organization } & 20\end{array}$

2 Literature review $\quad 22$

$2.1 \quad$ Fluid-structure interaction problem 25

$\begin{array}{lll}2.2 & \text { Immersed boundary method } & 27\end{array}$

3 Mathematical model $\quad 31$

3.1 Problem description 31

$\begin{array}{ll}3.2 \text { Governing equations } & 33\end{array}$

3.2.1 The 2-D planar flow 34

$\begin{array}{ll}3.2 .2 & \text { The axisymmetric flow } \\ 3.3\end{array}$

3.3 Boundary and initial conditions $\quad 41$

4 Numerical solution $\quad 44$

4.1 Finite element and immersed boundary methods 44

$\begin{array}{lll}4.2 & \text { Detailed formulation of } R_{m}^{\mathrm{f}^{\mathrm{B}}} & 46\end{array}$

4.2.1 The 2-D planar flow 46

4.2.2 The axisymmetric flow 48

$\begin{array}{lll}\text { 4.3 Solution of the transient problem } & 51\end{array}$

4.4 Solution of the nonlinear problem 51

4.5 Update of the membrane position X 52

4.6 Time-stepping scheme $\quad 52$

5 Results $\quad 54$

5.1 Validation: Simple shear flow 54

$\begin{array}{lll}5.2 & \text { Constricted channel flow } & 59\end{array}$

$\begin{array}{ll}5.3 & \text { Validation: Straight capillary flow } \\ 5.42\end{array}$

$\begin{array}{ll}5.4 \text { Constricted capillary flow } & 81\end{array}$

6 Conclusions and Future work $\quad 94$

$\begin{array}{lr}\text { Bibliography } & 97\end{array}$

$\begin{array}{ll}\text { A Appendix } & 103\end{array}$

A.1 Determination of $T_{1}$ for the 2-D planar flow 103 


\section{List of figures}

Figure 2.1 (a) Image, taken from a scanning electron microscope, of red and white blood cells, and platelets [18]. (b) Red blood cells under deformation while flowing in smaller capillaries. Scale bar is $5 \mu m[19]$.

Figure 2.2 Deformation of a microcapsule as it flows through a constricted capillary. Scale bar is $500 \mu m$ [8].

Figure 2.3 (a) Non-conforming mesh. (b) Conforming mesh. Adapted from Hou et al. [35].

Figure 2.4 (a) Immersed boundary. (b) Immersed domain [35].

Figure 2.5 Flow past an NACA0012 airfoil at $R e=5000$. (a) Pressure: IBM. (b) Pressure: body-fitted grid [37].

Figure 3.1 Sketch of the flow domain.

Figure 3.2 General membrane element. $\left(x_{1}, x_{2}, x_{3}\right)$ are fixed orthogonal coordinates and $\left(s_{1}, s_{2}, s_{3}\right)$ are moving curvilinear coordinates. $h$ is the membrane thickness.

Figure 3.3 Free-body diagram of the 1-D membrane element (gravity force neglected).

Figure 3.4 Normal stresses acting on the membrane element. Forces exerted on the membrane also included.

Figure 3.5 Initial prestress $T_{0}$ as a function of preinflation parameter $\alpha$ for SK, HK, and NHK membrane constitutive equations.

Figure 3.6 Boundary conditions for channel (or capillary) geometry. 41

Figure 4.1 Discretization of the membrane and fluid domains.

Figure 4.2 Given a global position, find the local coordinates.

Figure $4.3 \mathbf{t}_{\mathbf{1}}, \mathbf{n}$, and $\mathbf{e}_{\mathbf{r}}$ are unit tangent vector, unit normal vector, and unit radial vector, respectively.

Figure 4.4 An extra node is created to compute the line integral. 52

Figure 5.1 Simple shear flow domain.

Figure 5.2 Definition of $A, B$, and $\theta_{x y}$.

Figure 5.3 Capsule deformation for different grids at $\Gamma=0.0125$ and $R e=0.25$.

Figure 5.4 Steady-state flow structure and pressure field near the capsule at $R e=0.25$ and: (a) $\Gamma=0.0125$ and (b) $\Gamma=0.125$.

Figure 5.5 Temporal evolution of capsule deformation for different values of dimensionless shear stress $\Gamma$ and Reynolds number $R e$.

Figure 5.6 Temporal evolution of capsule orientation for different values of dimensionless shear stress $\Gamma$ at $R e=0.25$.

Figure 5.7 Temporal evolution of inlet pressure for different grids at $\bar{a}=1.63, \alpha=0.1, C a_{s}=0.05$, and $R e=1.0$.

Figure 5.8 Initial pressure field and capsule configuration at $\bar{a}=$ 1.63, $\alpha=0.1, C a_{s}=0.02$, and $R e=1.0$. 
Figure 5.9 Temporal evolution of the membrane and pressure field:

(a) $t=0.0001 \mathrm{~s}$; (b) $t=0.9233 \mathrm{~s}$; (c) $t=1.2 \mathrm{~s}$; and (d)

$t=1.9999 s$ at $\bar{a}=1.63, \alpha=0.1, C a_{s}=0.005$, and $R e=1.0$.

Figure 5.10 Temporal evolution of the membrane and pressure field:

(a) $t=0.0001 \mathrm{~s}$; (b) $t=0.88 \mathrm{~s}$; (c) $t=1.2 \mathrm{~s}$; and (d)

$t=1.9999 s$ at $\bar{a}=1.63, \alpha=0.1, C a_{s}=0.02$, and $R e=1.0$.

Figure 5.11 Temporal evolution of the membrane and pressure field:

(a) $t=0.0001 \mathrm{~s}$; (b) $t=0.8235 \mathrm{~s}$; (c) $t=1.2 \mathrm{~s}$; and (d) $t=1.9999 s$ at $\bar{a}=1.63, \alpha=0.1, C a_{s}=0.06$, and $R e=1.0$.

Figure 5.12 Temporal evolution of the membrane and pressure field:

(a) $t=0.0001 \mathrm{~s}$; (b) $t=0.7956 \mathrm{~s}$; (c) $t=1.2 \mathrm{~s}$; and (d)

$t=1.9999 s$ at $\bar{a}=1.63, \alpha=0.1, C a_{s}=0.1$, and $R e=1.0$.

Figure 5.13 (a) Temporal evolution of stress along membrane arclength at $\bar{a}=1.63, \alpha=0.1, C a_{s}=0.06$, and $R e=1.0$. (b) Position and membrane configuration at each time.

Figure 5.14 Stress colored-field at $\bar{a}=1.63, \alpha=0.1, C a_{s}=0.06$, and $R e=1.0$ : (a) $t \frac{\bar{V}}{L}=0.00$; (b) $t \frac{\bar{V}}{L}=0.18$; (c) $t \frac{\bar{V}}{L}=0.22$; (d) $t \frac{\bar{V}}{L}=0.33 ;$ and $(\mathrm{e}) t \frac{\bar{V}}{L}=0.44$.

Figure 5.15 Temporal evolution of inlet pressure at $\bar{a}=1.63, \alpha=0.1$, and $R e=1.0$ for different values of surface capillary number $C a_{s} .68$

Figure 5.16 Minimum lubricating film thickness at $\bar{a}=1.63, \alpha=0.1$, and $R e=1.0$ as a function of surface capillary number $C a_{s}$.

Figure 5.17 Temporal evolution of inlet pressure at $\bar{a}=1.63, C a_{s}=$ 0.06 , and $R e=1.0$ for different values of preinflation parameter $\alpha$.

Figure 5.18 Temporal evolution of inlet pressure at $\alpha=0.1, C a_{s}=$ 0.06 , and $R e=1.0$ considering different dimensionless capsule radii $\bar{a}$ and constriction ratios $\beta$.

Figure 5.19 Temporal evolution of capsule velocity at $\bar{a}=1.63$, $\alpha=0.1$, and $R e=1.0$ for different values of surface capillary number $C a_{s}$.

Figure 5.20 Mobility reduction factor $f$ as a function of $C a_{s}$ for different dimensionless capsule radii $\bar{a}$ and preinflation parameters $\alpha$ at $R e=1.0$.

Figure 5.21 Definition of $L_{x}, L_{y}$, and $L_{f r}$.

Figure 5.22 Temporal evolution of deformation $L_{f r}$ for different grids at $\bar{a}=0.8, \alpha=0.0, C a_{s}=0.24$, and $R e=1.0$.

Figure 5.23 Steady-state reached by the capsule at $\bar{a}=0.9, \alpha=0.0$, $C a_{s}=0.05$, and $R e=1.0$.

Figure 5.24 Temporal evolution of deformation $L_{x}$ for different values of $R e$ at $\bar{a}=0.9, \alpha=0.0$, and $C a_{s}=0.05$.

Figure 5.25 (a) Dimensionless $L_{x}$ and $L_{f r}$ as a function of $C a_{s}$. (b) Capsule velocity $u_{C G}$ as a function of $C a_{s}$ at $\bar{a}=0.9, \alpha=0.0$, and $R e=1.0$.

Figure 5.26 Comparison of capsule profiles at $\bar{a}=0.9, \alpha=0.0$, $R e=1.0$, for $C a_{s}=0.05$ and $C a_{s}=0.10$.

Figure 5.27 Dimensionless $T_{1}$ and $T_{2}$ as a function of meridional position $\phi$ at $\bar{a}=0.9, \alpha=0.0, C a_{s}=0.05$, and $R e=1.0$. 
Figure 5.28 Dimensionless $L_{x}, L_{y}$, and $L_{f r}$ as a function of $C a_{s}$ at $\bar{a}=0.8, R e=1.0$, for $\alpha=0.0$ and $\alpha=0.1$.

Figure 5.29 Comparison of capsule profiles at $\bar{a}=0.8, C a_{s}=0.24$, $R e=1.0$, for $\alpha=0.0$ and $\alpha=0.1$.

Figure 5.30 Comparison of capsule profiles at $\bar{a}=0.9, C a_{s}=0.05$, $R e=0.2$, for $\alpha=0.0$ and $\alpha=0.08$.

Figure 5.31 Comparison of capsule profiles at $\bar{a}=0.9, \alpha=0.0$, $C a_{s}=0.05$, and $R e=1.0$. Capsule membrane materials following Hooke's law, Skalak's, and Neo-Hookean equations.

Figure 5.32 Pressure field for capsules flowing through the straight capillary: (a) Skalak's membrane material. (b) Hooke's law. (c) Neo-Hookean membrane material at $\bar{a}=0.9, \alpha=0.0$, $C a_{s}=0.05$, and $R e=1.0$.

Figure 5.33 Temporal evolution of deformation $L_{f r}$ : (a) Different grids. (b) Different time steps at $\bar{a}=1.63, \alpha=0.1, C a_{s}=0.05$, and $R e=1.0$.

Figure 5.34 Initial pressure field and capsule configuration at $\bar{a}=$ 1.63, $\alpha=0.1, C a_{s}=0.02$, and $R e=1.0$.

Figure 5.35 Temporal evolution of the membrane and pressure field:

(a) $t=0.0001 \mathrm{~s}$; (b) $t=0.6013 \mathrm{~s}$; (c) $t=0.82 \mathrm{~s}$; and (d) $t=1.4 s$ at $\bar{a}=1.63, \alpha=0.1, C a_{s}=0.005$, and $R e=1.0$.

Figure 5.36 Temporal evolution of the membrane and pressure field:

(a) $t=0.0001 \mathrm{~s}$; (b) $t=0.5546 \mathrm{~s}$; (c) $t=0.82 \mathrm{~s}$; and (d) $t=1.4 s$ at $\bar{a}=1.63, \alpha=0.1, C a_{s}=0.02$, and $R e=1.0$.

Figure 5.37 Temporal evolution of the membrane and pressure field:

(a) $t=0.0001 \mathrm{~s}$; (b) $t=0.5004 \mathrm{~s}$; (c) $t=0.82 \mathrm{~s}$; and (d) $t=1.4 s$ at $\bar{a}=1.63, \alpha=0.1, C a_{s}=0.06$, and $R e=1.0$.

Figure 5.38 Temporal evolution of the membrane and pressure field:

(a) $t=0.0001 \mathrm{~s}$; (b) $t=0.4747 \mathrm{~s}$; (c) $t=0.82 \mathrm{~s}$; and (d) $t=1.35 s$ at $\bar{a}=1.63, \alpha=0.1, C a_{s}=0.1$, and $R e=1.0$.

Figure 5.39 Temporal evolution of the capsule shape: (a) $t=$ $0.0001 \mathrm{~s}$; (b) $t=0.5546 \mathrm{~s}$; (c) $t=0.82 \mathrm{~s}$; and (d) $t=1.4 \mathrm{~s}$ at $\bar{a}=1.63, \alpha=0.1, C a_{s}=0.02$, and $R e=1.0$.

Figure 5.40 (a) Temporal evolution of membrane stress $T_{1}$ along angular position $\phi$. (b) Temporal evolution of membrane stress $T_{2}$ along angular position $\phi$. (c) Capsule position at $\bar{a}=1.63$, $\alpha=0.1, C a_{s}=0.06$, and $R e=1.0$.

Figure 5.41 Temporal evolution of inlet pressure at $\bar{a}=1.63, \alpha=0.1$, and $R e=1.0$ for different values of surface capillary number $C a_{s} .89$

Figure 5.42 Minimum lubricating film thickness at $\bar{a}=1.63, \alpha=0.1$, and $R e=1.0$ as a function of surface capillary number $C a_{s}$.

Figure 5.43 Temporal evolution of inlet pressure at $\bar{a}=1.63, C a_{s}=$ 0.06 , and $R e=1.0$ for different values of preinflation parameter $\alpha$.

Figure 5.44 Temporal evolution of inlet pressure at $\alpha=0.1, C a_{s}=$ 0.06 , and $R e=1.0$ considering different dimensionless capsule radii $\bar{a}$. 
Figure 5.45 Capsule velocity along the $x$-axis for different values of surface capillary number $C a_{s}$. At $\bar{a}=1.63, \alpha=0.1$, and $R e=1.0 .91$

Figure 5.46 Maximum capsule velocity as a function of surface capillary number $C a_{s}$ for different membrane materials. At $\bar{a}=1.63$, $\alpha=0.1$, and $R e=1.0$.

Figure 5.47 Mobility reduction factor $f$ as a function of $C a_{s}$ for different membrane materials, dimensionless capsule radii $\bar{a}$, and preinflation parameters $\alpha$ at $R e=1.0$. 


\section{List of tables}

Table 5.1 Values of parameters for the constricted channel flow. $\quad 61$

Table 5.2 Values of parameters for the straight capillary flow. $\quad 73$

Table 5.3 Values of parameters for the constricted capillary flow. 81 


\section{List of Abreviations}

\begin{tabular}{|c|c|c|}
\hline$a$ & - & capsule radius \\
\hline$a_{0}$ & - & unstressed capsule radius \\
\hline $\bar{a}$ & - & dimensionless capsule radius \\
\hline$A, B$ & - & maximum and minimum axes of the capsule \\
\hline BIM & - & boundary integral method \\
\hline$C a$ & - & capillary number for droplet flow \\
\hline$C a_{s}$ & - & surface capillary number for capsule flow \\
\hline$D_{x y}$ & - & dimensionless capsule deformation \\
\hline$e$ & - & solid element \\
\hline $\mathbf{e}_{\mathbf{r}}$ & - & unit radial vector \\
\hline$E$ & - & Young's modulus \\
\hline$f$ & - & mobility reduction factor \\
\hline$f_{e}$ & - & fluid element \\
\hline f & - & force density \\
\hline $\mathrm{f}^{\mathrm{B}}$ & - & body force \\
\hline FDM & - & finite difference method \\
\hline FEM & - & finite element method \\
\hline FSI & - & fluid-structure interaction problem \\
\hline$G$ & - & material shear modulus \\
\hline$G_{s}$ & - & surface shear modulus \\
\hline$h$ & - & membrane thickness \\
\hline$h_{\min }$ & - & minimum film thickness \\
\hline$H_{s}$ & - & maximum half channel height \\
\hline$H_{0}$ & - & minimum half channel height \\
\hline
\end{tabular}




\begin{tabular}{|c|c|c|}
\hline$H(x)$ & - & channel wall function \\
\hline HK & - & Hooke's law (Linear elastic materials) \\
\hline $\mathbf{I}$ & - & Identity tensor \\
\hline IBM & - & immersed boundary method \\
\hline $\mathbf{J}, \mathbf{M}$ & - & Jacobian and mass matrixes \\
\hline$k$ & - & shear rate \\
\hline$k_{i}$ & - & Newton-Raphson iteration index \\
\hline$k_{n}$ & - & solid domain node \\
\hline$k_{t}$ & - & time index \\
\hline$l_{s}(t)$ & - & length along the $s_{1}$-direction \\
\hline$L$ & - & characteristic length \\
\hline$L_{x}, L_{y}, L_{f r}$ & - & capsule deformation metrics \\
\hline $\max ()$ & - & function maximum \\
\hline $\mathbf{n}$ & - & unit normal vector \\
\hline$n_{L}$ & - & number of Lagrangian points \\
\hline$N E X, N E Y$ & - & number of elements along horizontal and vertical directions \\
\hline NHK & - & Neo-Hookean model \\
\hline$p$ & - & pressure scalar field \\
\hline$p_{\text {in }}, p_{\text {out }}$ & - & inlet and outlet pressure \\
\hline $\mathbf{R}, \mathbf{S v}$ & - & residual and solution vectors \\
\hline$R e$ & - & Reynolds number \\
\hline$R_{s}$ & - & inlet radius of capillary \\
\hline$R_{0}$ & - & constriction radius of capillary \\
\hline$R_{1}, R_{2}$ & - & principal radii of curvature \\
\hline$R(x)$ & - & capillary wall function \\
\hline $\mathrm{RBC}$ & - & red blood cells \\
\hline$s_{i}$ & - & arc-length along $i$-direction \\
\hline$s_{i \infty}$ & - & unstressed arc-length along $i$-direction \\
\hline SK & - & Skalak's model \\
\hline
\end{tabular}




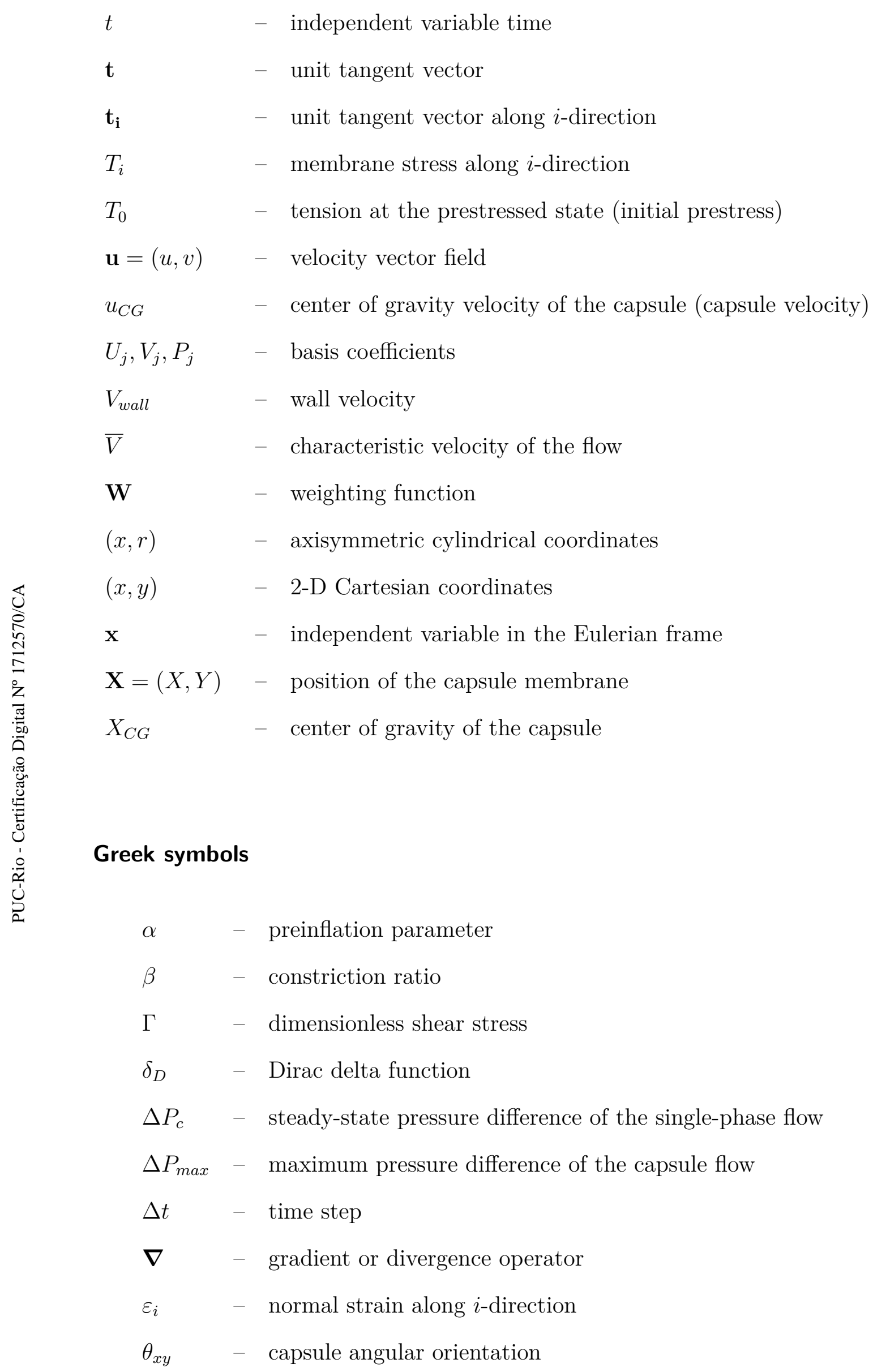




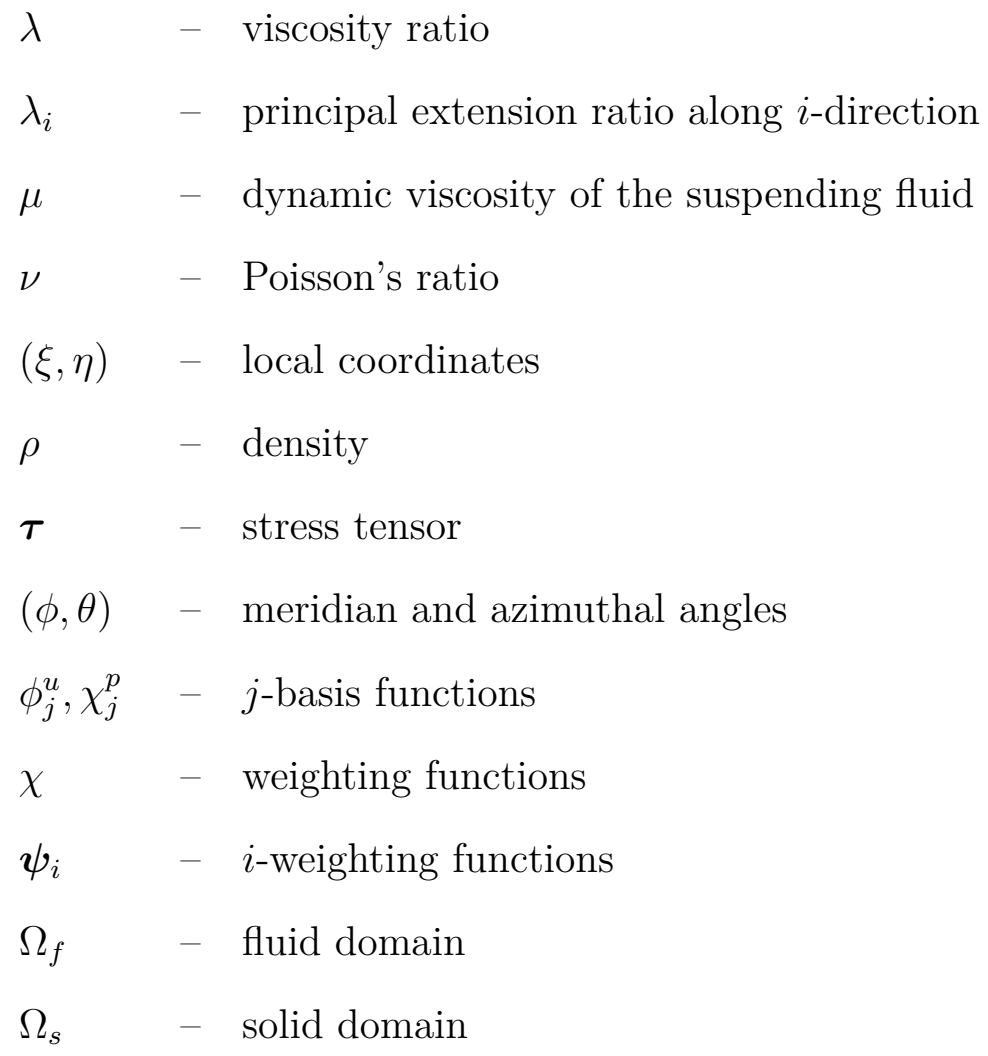




\section{Introduction}

The present work focuses on the numerical study of the incompressible flow of a suspended capsule in a Newtonian fluid through two different geometries: a planar constricted microchannel and an axisymmetric constricted microcapillary.

A capsule is a closed fluid-filled membrane made by an elastic material [1]. Flow of suspended capsules can be found in different natural and industrial applications, including transport of red blood cells (RBC) on hemodynamics [2,3], cell manipulation in microfluidic devices [4], targeted delivery of active substances $[5,6,7]$, flow through porous media $[8,9]$, among others. The fundamental understanding of the flow of suspended microcapsules and the effect of different parameters on capsule deformation and liquid flow is important to gain better control of such processes.

Flow through porous media is of special importance for this work. In this application, capsules are injected to block water flow, altering the main flow path. In other words, capsule suspension may alter water mobility as it flows through pore throats, blocking preferential flow paths, and diverting water to oil containing pores, resulting in an increase in the volume of oil recovered $[8,9]$.

The assessment of the microscale phenomena at the pore-level is fundamental to understand the macroscopic dynamics. In their work, Øren \& Bakke [10] estimated different properties of porous media, such as permeability and conductivity, by reconstructing 3-D porous media utilizing 2-D thin section images. The authors utilized an equivalent network model to represent the reconstructed rock sample. In network models, larger pores are connected by smaller pores. In other words, pore bodies are linked by pore throats. In this work, a single pore throat connecting two adjacent pore bodies is studied and modeled as constricted microscale geometries: channel and capillary.

Capsule dynamics in viscous flow poses a non-trivial problem due to its complex fluid-solid dynamics. A significant number of experimental and nu- 
merical studies have been conducted to understand the fundamental physical phenomenon, and have been reviewed by Barthès-Biesel [11].

Due to its microscale, capsule flow is extremely challenging to study experimentally for a wide range of operating conditions. For that reason, numerical simulations are conveniently utilized. There are different numerical methods for addressing fluid-structure interaction problems (FSI), which usually consume high computational resources to solve both dynamic equations, fluid and solid, on adapting or evolving grids.

Among different methods, the immersed boundary method (IBM) was selected in the present study. This method, originally developed by Peskin [12], was utilized to study flow patterns around heart valves, by modeling flexible characteristics of a heart fiber as a flexible structure immersed in a fluid domain.

Immersed boundary method utilizes an Eulerian-Lagrangian description, at which the solid phase is considered as a part of the fluid domain where additional forces are exerted, and its position is determined by a Lagrangian variable associated with a fixed coordinate system. Thus, the interaction of the solid on the fluid and vice versa configures a two-way interaction problem.

The following chapters will discuss in further details state-of-the-art, mathematical modeling, numerical methodology, and results obtained throughout this work.

\section{1}

\section{Objectives}

The main objective of this work is to study the incompressible flow of a suspended capsule in a Newtonian fluid through a constricted microchannel and microcapillary.

The specific goals include:

- Develop a mathematical formulation of coupled fluid flow and elastic capsule dynamics;

- Validate formulation and numerical implementation for two different geometries: simple shear flow and straight capillary flow; 
- Analyze the effect of different membrane constitutive equations on the mechanical response of a capsule as it flows through a constriction;

- Evaluate capsule configuration (profile, deformation, and the like), pressure difference, membrane stresses, and other flow characteristics as a function of capsule radius, prestressed condition, constriction ratio, capsule membrane material, and flow conditions;

- Evaluate the reduction of the continuous phase mobility as a function of capsule radius, prestressed condition, and capsule membrane material.

The determination of the relationship between flow rate and pressure difference due to the presence of a suspended capsule through a constriction is of paramount importance to assess blocking efficiency of capsule flow in porous media. Therefore, this study will contribute in the design of microcapsules that can be used to divert the preferential water path in oil displacement process in porous media.

\section{2}

\section{Thesis organization}

This document has been divided into five additional chapters. The following chapter presents experimental studies and different numerical methodologies used to address fluid-structure interaction problems, including the one selected for this work, the immersed boundary method.

Chapter 3 describes the problem to be studied, and lists the basic assumptions. Conservation of mass and momentum equations are presented, with special interest in the fluid-solid coupling. Mechanical behavior of the membrane is addressed considering two configurations, a 2-D Cartesian and an axisymmetric configuration. Finally, boundary and initial conditions are presented.

The numerical methodology is discussed in Chapter 4. In this work, the transient nonlinear problem has been solved numerically by the finite element method (FEM) coupled with the immersed boundary method (IBM). Nonlinearities have been addressed by the Newton-Raphson method, and time discretization has utilized a predictor-corrector algorithm.

Chapter 5 discusses the results obtained in a 2-D Cartesian coordinate system for simple shear flow and constricted channel flow. In addition, the 
validation of formulation and implementation of the axisymmetric flow solver is presented. Then, constricted axisymmetric capillary flow is analyzed. Time evolution of membrane profile, membrane stress, pressure difference, among others are the object of interest, and their dependence on capsule radius, prestressed condition, membrane material, etc.

Final remarks are discussed in Chapter 6 as well as suggestions for future work to improve the code implementation and extend the analysis. 


\section{Literature review}

This chapter is devoted to review different studies on capsule flow through confined geometries. In general, capsules are liquids or gels enclosed by a thin elastic membrane, if subjected to large strain, the membrane may break up or deform plastically [13]. There is a myriad of microscale applications of capsules, from natural to industrial, including hemodynamics $[2,3,5,14,15]$, food industry $[16,17]$, flow in porous media $[8,9]$, among others. In most of these applications, due to small dimensions involved, e.g. microchannels and microcapillaries, the interaction between a flowing capsule and the surrounding fluid poses a complex problem. Over the years, several experimental and numerical studies were performed to understand how different parameters play an important role in capsule dynamics. Some of such studies will be discussed.

Blood and suspended cells flowing in an organic system have been studied by many authors, as reviewed by Freund [15]. Blood is a complex system composed by red blood cells, white blood cells, and the like, flowing through an intricate network of vessels and small capillaries, as shown in Fig. 2.1. Red blood cells are composed by a thin lipid bilayer membrane, and can be modeled as a thin shell model. Furthermore, deformability of red blood cells receives special attention since it affects the optimal gas exchange between blood and tissues [2]. In fact, some diseases can modify cell membrane stiffness affecting circulation and the mechanical response through constrictions. As extensively reviewed by Mokken et al. [14], malaria, renal failure, among other diseases are related to the decrease of cell deformability. For instance, red blood cells infected with malaria parasites presented less deformability when aspirated into a micropipette.

In the context of RBC simulations, Freund [15] highlighted the importance of the appropriate coupling between the flow solver and the solid mechanics, including the membrane constitutive model. For instance, in blood flow, cells can be subjected to large deformation, hence the fluid-solid coupling requires special attention to avoid significant error and numerical instabilities. 
(a)

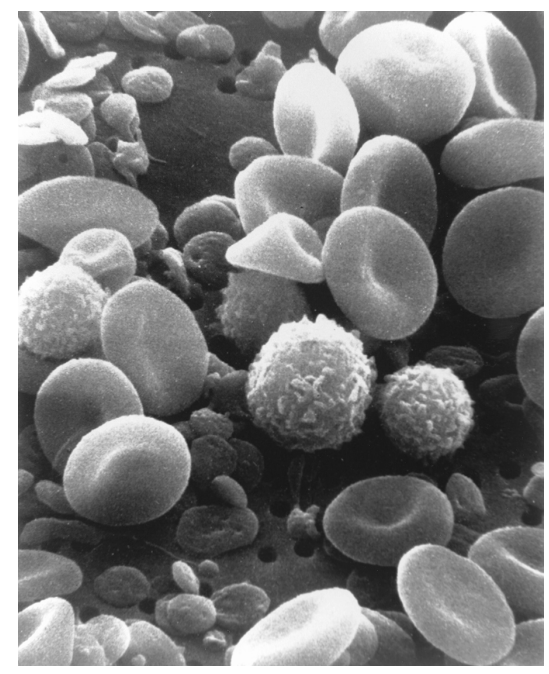

(b)

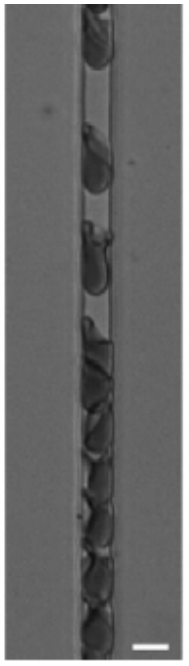

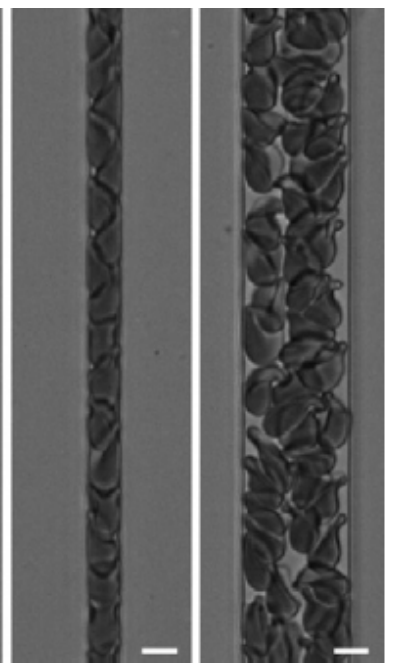

Figure 2.1: (a) Image, taken from a scanning electron microscope, of red and white blood cells, and platelets [18]. (b) Red blood cells under deformation while flowing in smaller capillaries. Scale bar is $5 \mu m$ [19].

Drugs encapsulated in red blood cells have important medical applications as a targeted vascular delivery of therapeutic agents. In this case, these cells are intravascular carriers of therapeutic enzymes, antibiotics, anti-viral drugs, anti-inflammatory drugs, anti-cancer agents, and other encapsulated drugs [5].

Enhanced oil recovery is an area of application of special importance for this work. Do Nascimento et al. [8] discussed potential applications of capsule flow through porous media in controlling aqueous phase flow mobility by designing capsules with appropriate mechanical properties. Capsules with different radii, thicknesses, and materials (ratio of cross-link) were fabricated to determine the effect of these variables on the mechanical response to compression in a quasi-static test. Additionally, tests included capsule flow through a constricted capillary. In such tests, three different capsule regimes were observed during the flow through a constriction: predeformation, maximum deformation, and recovery, as presented in Fig. 2.2. They noted that pressure difference in time builds up as the capsule goes through narrower regions. Thus, the macroscopic flow behavior is related to the microscopic flow dynamics at the pore throat scale, which is modeled as constricted microcapillaries, giving a better understanding of pore blocking mechanism in porous media.

Flow of suspended capsules in different flow geometries has been widely studied. An elastic capsule under simple shear flow, for instance, has been 


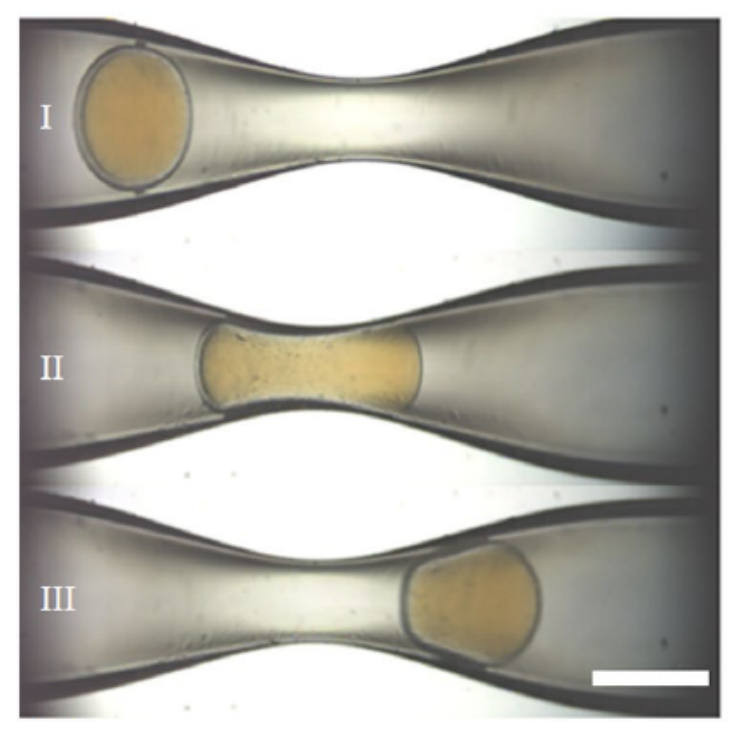

Figure 2.2: Deformation of a microcapsule as it flows through a constricted capillary. Scale bar is $500 \mu m$ [8].

addressed by experiments and numerical analyses [20, 21, 22, 23, 24, 25]. These researchers revealed the effect of capsule mechanical properties and flow conditions on deformation, inclination angle, tank treading frequency of the capsule, and the flow structure around it.

For years, determining the mechanical properties of small capsules has been the objective of different researchers. Rachik et al. [26] conducted compression tests in order to characterize mechanical properties of capsules, and to assess the limits of the thin shell theory. On the other hand, Lefebvre et al. [27] proposed a dynamic method for characterization based on visualization of capsule deformation in microchannels of similar dimensions, as a function of flow conditions. Then, the capsule elastic modulus was determined by defining an inverse problem together with numerical modeling.

Capsule dynamics depends on different parameters, including the ratio of viscous to elastic forces, defined as the surface capillary number $C a_{s}$ :

$$
C a_{s}=\frac{\text { viscous force }}{\text { elastic force }}=\frac{\mu \bar{V}}{G_{s}},
$$

where $\mu$ is the viscosity of the suspending fluid, $\bar{V}$ is the characteristic velocity of the flow, and $G_{s}$ is the surface shear modulus expressed in units of Pa.m, which depends on membrane elastic material and thickness. High surface 
capillary number indicates that viscous force dominates when compared to elastic force, leading to a highly deformed capsule. Notice that, the surface capillary number $C a_{s}$ is analogous to the capillary number $C a$ for droplet flow, in which two immiscible fluids share an interface between them.

Risso et al. [28] studied experimentally the motion and the deformation of bioartificial capsules in millimetric straight tubes at low to moderate surface capillary numbers $\left(C a_{s} \leq 0.125\right)$, in Stokes flow. The Stokes flow is justified because inertial effects are neglected, due to small velocities and dimensions presented in such problems. Capsules reached steady-state conditions depending on $C a_{s}$ and the ratio of capsule to tube radii. They found that at small to moderate $C a_{s}$, the stiffer capsule is rounded at both ends. The rear curvature of the membrane flattens and the axial length increases as $C a_{s}$ rises. The authors realized that their results differed quantitatively from numerical predictions, probably due to a different membrane mechanical model and no initial preinflation were considered.

Capsule deformation in microchannels of comparable dimensions was also used by Lefebvre et al. [27] to evaluate the mechanical properties of the suspended capsule as a function of flow conditions. Gupspun et al. [29] studied the flow of soft microcapsules in capillaries using particle tracking velocimetry and numerical simulations.

Changes in microchannel cross-section may have an important effect on capsule dynamics, as experimentally observed by Leclerc et al. [30]. The authors studied the flow through converging-diverging channels. They estimated the membrane shear modulus from relaxation process of the capsule as it flows out of the constriction.

Due to small scales, experimental studies of flow of capsules are extremely challenging, thus numerical approaches arise to complement them by exploring different geometries and a broad range of operating parameters.

\section{1}

\section{Fluid-structure interaction problem}

In fluid-structure interaction (FSI) problems, a solid phase is deformed by the boundary forces exerted by the fluid flow, changing the flow boundaries, and thus directly affecting the flow pattern. This configures a complex prob- 
lem, which in general lacks an analytical solution. For that reason, numerical modeling appears as an important tool to explore different geometries and operating parameters, and therefore to unveil the physical phenomenon.

In order to solve FSI problems, different approaches have been developed over time. Generally speaking, most approaches require high computational resources to couple fluid and solid dynamics on adaptable or evolving grids.

As already discussed, because of small dimensions and velocities, in many cases, inertial effects are neglected, i.e. Stokes flow, which is suitable for the boundary integral method (BIM). Hu et al. [31] coupled BIM and finite element to analyze suspended capsule flow through channels with circular and square cross-sections. Mechanical properties were estimated by comparing numerical predictions with experiments. Furthermore, Leyrat-Maurin \& Barthès-Biesel [32] also studied a capsule in Stokes flow. They studied capsule evolution when flowing through a hyperbolic constriction. The authors presented the time evolution of capsule configuration, center of gravity of the capsule, its velocity, membrane stresses, among other variables, in order to provide quantitative data to assess red blood cell filtration experiments.

Dimitrakopoulos \& Kuriakose [33] developed a methodology, based on numerical simulations, to determine the membrane shear modulus $G_{s}$ independently of its area-dilation modulus by studying strain-hardening capsules in a converging microcapillary in Stokes flow. The authors compared the numerical results to experimental measurements of the capsule deformation.

Inertial effects, i.e. finite Reynolds number $R e$, were explored by Carroll [13], who numerically studied suspended droplet and capsule flows, using Volume-of-Fluid front tracking method and finite difference method (FDM) in straight capillaries, and transient flow in channels with expansions and contractions. The mechanical behavior of the capsule membrane was described by two different constitutive models: Neo-Hookean, which presents strain-softening behavior and is often found in biological membranes; and the Skalak's model, which shows a strain-hardening response. The author found that above a critical $C a_{s}$, no stable capsule shape could be found.

Rorai et al. [1] studied capsule flow through a constricted microchannel by using a hybrid boundary integral-mesh method [34]. They discussed that constrictions are considered as models of arterial vessels in physiological sys- 
tems. These vessels eventually can get narrowed, a serious medical condition called stenosis. The authors also neglected inertial effects. The capsule was modeled by a 2-D elastic membrane, using the Neo-Hookean constitutive model. Inner and outer fluids shared the same density and viscosity. Initial condition for the capsule configuration was the steady-state solution in the flow through a straight channel, in order to independently assess the constriction effect. They noted that stiffer capsules remained nearly spherical. However, as $C a_{s}$ increases, capsules may adopt other shapes, including bulletlike or croissant-like shapes. Furthermore, they reported capsule deformation, retention time, velocity, and membrane stress as a function of a wide range of $C a_{s}$ and constriction ratio, with a special focus on fractional change of the capsule surface area along the axis at different $C a_{s}$. As expected, the capsule accelerates as it reaches the converging part of the channel, reaching its maximum velocity at the center of the constriction. The authors also proposed that if the variation of the capsule surface area was not easily measured, the projected perimeter in the plane was a suitable option to address membrane deformability.

Thus, many of the reviewed research focused on flows with negligible inertial effects, moderate surface capillary number $C a_{s}$ (ranging from $10^{-2}$ to 1), and moderate capsule deformation. Moreover, in most cases, the main goal was to evaluate the capsule deformation to estimate membrane mechanical properties, and did not fully discuss the pressure response of the capsule flow, which in this study is essential to understand the capsule blocking mechanism.

\section{2}

\section{Immersed boundary method}

In their review, Hou et al. [35] revisited several numerical approaches and mesh treatments for FSI problems with incompressible flows for different engineering applications. The first approach, named as monolithic approach, considers that fluid and structure dynamics are described in a mathematical framework, which is solved in a single algorithm. The other approach known as partitioned approach, solves separately both fluid and solid dynamics coupling explicitly by interface conditions, requiring time-tracking of interface position and its related quantities. The different methods can also be classified according to the mesh treatments, as conforming mesh or non-conforming mesh. In the latter, the boundary location and related interface conditions are enforced as constraints on the solution of the fluid-solid equations within 
their respective grids. In the conforming mesh methods, the mesh conforms the fluid-solid interface and interfacial conditions are applied along element boundaries, requiring mesh-updating as solution evolves. Both treatments are illustrated in Fig. 2.3.

(a)

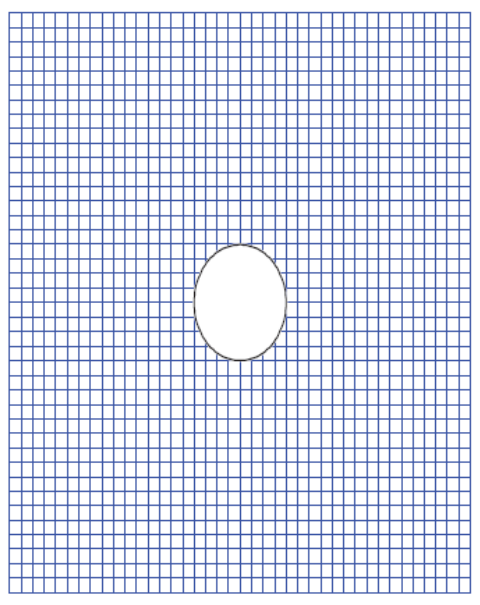

(b)

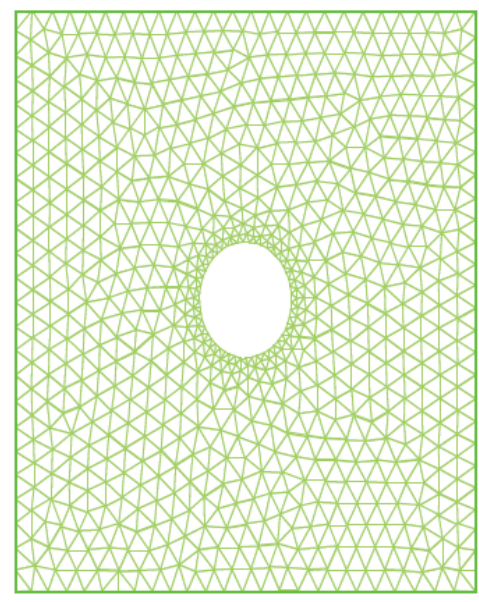

Figure 2.3: (a) Non-conforming mesh. (b) Conforming mesh. Adapted from Hou et al. [35].

A solid phase immersed in a fluid flow can also be modeled using a class of FSI methods named immersed methods, as discussed by Hou et al. [35]. The immersed body can be treated as a boundary itself or a boundary with a finite domain enclosed. Thus, in principle, two different types of immersed methods can be defined: the immersed boundary method and the immersed domain method, as shown in Fig. 2.4. In applications in which the volume occupied by the body is taken into account, the immersed domain is utilized. The idea is to define an artificial fluid inside the enclosed region so the fluid domain can be extended to the whole computational domain. Thus, the interaction force between both phases needs to be imposed at the interface as well as inside the artificial fluid domain.

In this work, the immersed boundary method (IBM) is used to describe the membrane deformation and the finite element method to solve the flow governing equations [36]. Immersed methods belong to the non-conforming mesh class of methods, in which a force term is added to the equations of fluid dynamics to account for fluid-structure interaction. It was developed in the early seventies by Peskin [12] to study flow patterns around heart valves by modeling a heart fiber as a flexible boundary immersed in a fluid domain. Peskin initially used the finite difference method to discretize the differential equations. Since then, IBM has been modified and employed in different 
(a)

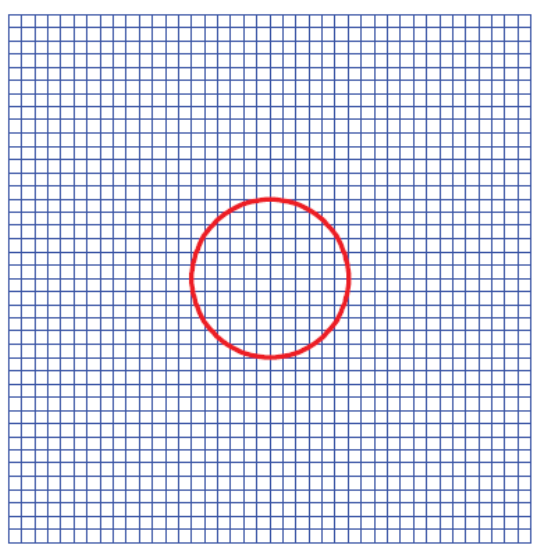

(b)

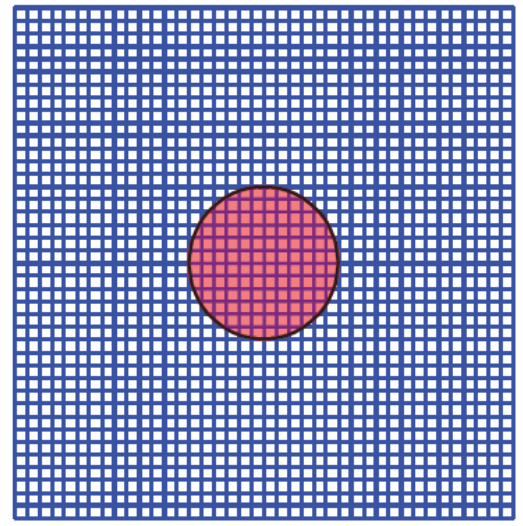

Figure 2.4: (a) Immersed boundary. (b) Immersed domain [35].

applications, such as flow around rigid bodies [37, 38, 39]. For instance, Ilinca \& Hétu [37] used a 3-D finite element immersed boundary method, together with a level set function. Comparisons with body-fitted grid solutions showed good agreement for different applications, including flow in an obstructed channel, flow around spheres, predicted Strouhal number for the periodic flow, and the well known flow past an NACA0012 airfoil, as illustrated in Fig. 2.5. Mittal \& Iaccarino [40] presented a comprehensive review on the method.

(a)

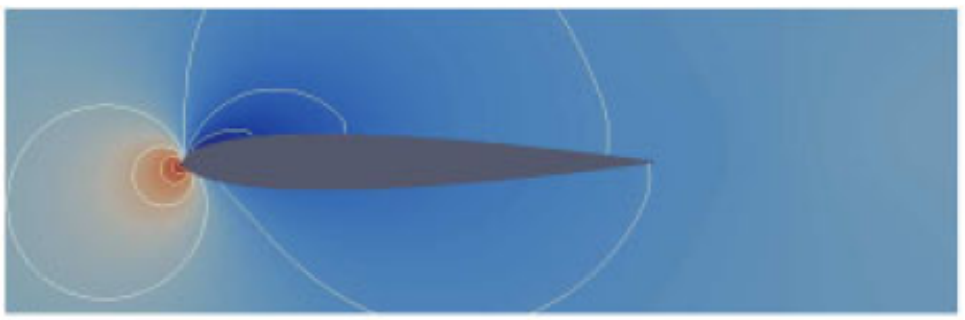

(b)

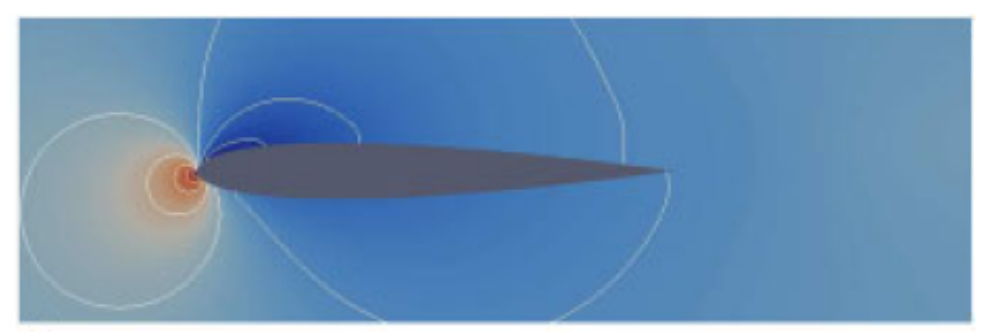

Figure 2.5: Flow past an NACA0012 airfoil at $R e=5000$. (a) Pressure: IBM. (b) Pressure: body-fitted grid [37].

IBM utilizes an Eulerian-Lagrangian description. The flow field is described using an Eulerian approach in a fixed grid that does not conform with the solid boundary. The solid phase, described using a set of Lagrangian variables, moves through the Eulerian grid, and it is considered as a part of the fluid domain, where additional forces are included in the fluid momentum 
equation. For that reason, solid-fluid interaction is described by an additional body force that is different than zero only along the membrane. A singular vector field represents the body force defined mathematically by the Dirac delta function $\delta_{D}$; therefore, only acting on the interface.

The finite element method is utilized leading to no need for approximating the Dirac delta function, similarly to the variational formulation developed by Boffi \& Gastaldi [36]. Thus, the capsule effect on fluid dynamics is computed through evaluating weighting functions at the Lagrangian points, whilst the capsule position is advected by the fluid velocity field, defining a two-way interaction problem.

Therefore, in this study, the problem is solved using a fixed nondeforming grid for the flow (avoiding continuous grid generation at each time), and a moving and deforming grid for the membrane. Furthermore, not only considers inertial effects but also explores a wide range of parameters, including lower surface capillary number $C a_{s}$. 


\section{3}

\section{Mathematical model}

This chapter presents the mathematical modeling of the capsule flow through a constricted channel and a constricted capillary. The transient nonlinear problem is described by the Navier-Stokes equations for incompressible flows, the membrane equilibrium equation, and the constitutive material equation of the membrane with the appropriate boundary and initial conditions. The objective is to assess the suspended capsule flow through the constricted geometry and determine the effect of capsule radius, membrane material, flow conditions, and the like on the flow behavior.

\section{1}

\section{Problem description}

A capsule suspended in a liquid phase flows through a constricted channel (or capillary), as sketched in Fig. 3.1. The flow domain is bounded by synthetic inlet and outlet planes, the symmetry plane (or symmetry axis), and the channel (or capillary) wall, described by a cosine function:

$$
H(x)=\frac{H_{s}+H_{0}}{2}+\frac{H_{s}-H_{0}}{2} \cos \left(2 \pi \frac{x}{L}\right)
$$

where $L$ is the channel length, $H_{s}$ and $H_{0}$ are the maximum and minimum half channel heights, respectively. Let $\beta \equiv H_{0} / H_{s}$ be the constriction ratio.

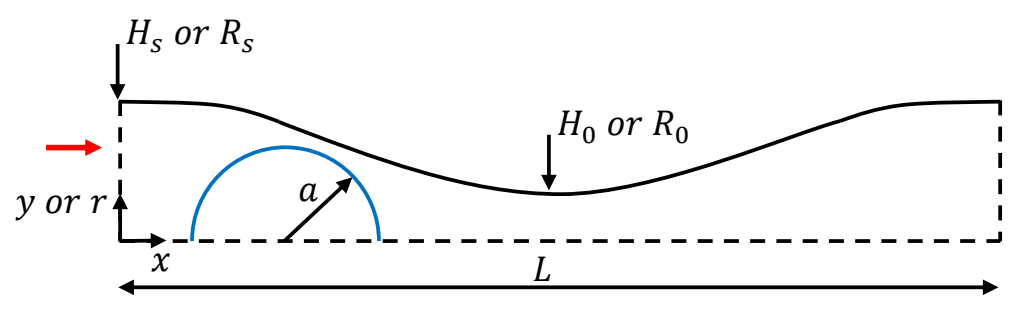

Figure 3.1: Sketch of the flow domain.

Similar to eq. (3-1), an expression $R(x)$ for the axisymmetric flow can be obtained considering that $R_{s}$ and $R_{0}$ are used for maximum and minimum 
capillary radii, respectively.

The following basic assumptions are utilized in this work:

- There is no density difference between inner and outer phases, thus a symmetric flow is expected. For the 2-D planar flow, a symmetry plane at the bottom side. For the axisymmetric flow, a symmetry axis;

- Fully developed flow at the inlet and outlet planes. For instance, at the inlet plane, a long enough straight channel (or capillary) can be considered upstream from the studied domain;

- Incompressible flows;

- Isothermal process, thus properties such as density or viscosity are constants in each phase.

- No gravity effects are considered.

Regarding the capsule, in order to apply the membrane theory ${ }^{1}$, the following simplifications are required:

- Capsule thickness is negligible when compared to other dimensions;

- Isotropic and homogeneous membrane material;

- Stress normal to the capsule surface is small when compared to stresses along other directions.

Since twisting and bending moments are small enough to be neglected, and no shear stress is considered, the membrane is only subjected to stretching and compression.

Therefore, the 2-D planar channel (or axisymmetric capillary) flow and the thin capsule membrane are described using a 2-D Cartesian (or cylindrical) coordinate system, defined by the flow domain $\Omega_{f}$ (black line in Fig. 3.1). The thin capsule defines a solid domain $\Omega_{s}$ (blue line in Fig. 3.1), and consists of a 1-D massless fiber-like flexible structure, defined in a Lagrangian frame system.

${ }^{1}$ Sometimes the "membrane theory" is referred to as the "membrane theory of shells" [41]. 


\section{2}

\section{Governing equations}

For incompressible viscous flows, the momentum and mass conservation equations, in an Eulerian frame, are written as

$$
\begin{aligned}
\rho\left(\frac{\partial \mathbf{u}}{\partial t}+\mathbf{u} \cdot \nabla \mathbf{u}\right) & =\boldsymbol{\nabla} \cdot \boldsymbol{\tau}+\mathbf{f}^{\mathbf{B}} \\
\boldsymbol{\nabla} \cdot \mathbf{u} & =0
\end{aligned}
$$

where $t, \rho, \mathbf{u}=(u, v), \boldsymbol{\tau}$, and $\mathbf{f}^{\mathbf{B}}$ are the time, density, velocity vector, stress tensor, and body force (per unit volume) associated with the fluid-solid interaction, respectively. For a Newtonian liquid, the stress tensor is defined as

$$
\boldsymbol{\tau}=-p \mathbf{I}+\mu\left[\boldsymbol{\nabla} \mathbf{u}+(\boldsymbol{\nabla} \mathbf{u})^{T}\right]
$$

where $p$ and $\mu$ are the pressure and viscosity, respectively, and $\mathbf{I}$ is the identity tensor.

As mentioned before, the interaction between an immersed solid structure, i.e. the capsule membrane, and a fluid can be modeled through the body force (per unit volume) $\mathbf{f}^{\mathrm{B}}$ in the momentum equation, which represents the force that the membrane exerts on the fluid. It is described as a volume integral over the solid volume $\Omega_{s}[12]$ as follows:

$$
\mathbf{f}^{\mathbf{B}}(\mathbf{x})=\int_{\Omega_{s}} \mathbf{f} \delta_{D}(\mathbf{x}-\mathbf{X}) d \Omega_{s}^{\prime}
$$

where $\mathbf{X}$ is an independent variable in the Eulerian frame, $\mathbf{X}$ is the position of solid domain, $\mathbf{f}$ is the force density exerted on the fluid by the membrane, and $\delta_{D}$ is the Dirac delta function. Hence the body force $\mathbf{f}^{\mathbf{B}}$ is a singular vector field, zero everywhere except over the membrane.

In a general 3-D problem, coordinates of the Lagrangian points of the 
membrane $\mathbf{X}$ can be written as $\mathbf{X}=\mathbf{X}\left(s_{1}, s_{2}, s_{3}, t\right)$, where $\left(s_{1}, s_{2}, s_{3}\right)$ are the coordinates defined in a suitable curvilinear system attached to the membrane, as sketched in Fig. 3.2. Notice that, the stress in the $s_{3}$-direction is small when compared to the ones along $s_{1}$ and $s_{2}$ directions. In fact, these two last stresses are assumed to be constant across the membrane thickness $h$.

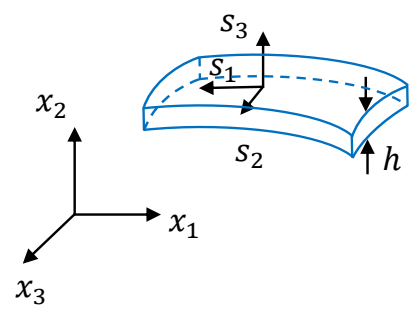

Figure 3.2: General membrane element. $\left(x_{1}, x_{2}, x_{3}\right)$ are fixed orthogonal coordinates and $\left(s_{1}, s_{2}, s_{3}\right)$ are moving curvilinear coordinates. $h$ is the membrane thickness.

As already mentioned, in this work two different problems are addressed: a 2-D planar flow and an axisymmetric flow.

\subsection{1}

\section{The 2-D planar flow}

In the 2-D planar flow, membrane points $\mathbf{X}$ are written as $\mathbf{X}=\mathbf{X}\left(s_{1}, t\right)$, where $s_{1} \in\left[0, l_{s}(t)\right]$ is the coordinate along the capsule membrane. The length of the membrane $l_{s}(t)$ changes with time as the capsule flows.

In order to obtain $\mathbf{f}$, a free-body diagram of the membrane element is drawn in Fig. 3.3, including only relevant forces along the $s_{1}$-direction and the force exerted on the membrane element by the surrounding fluid phase $\mathbf{F}_{\text {fluid/solid }}$.

Thus, the net force along the $s_{1}$-direction acting on the surface $h \times 1$ is

$$
\left(T_{1}+\frac{\partial T_{1}}{\partial s_{1}} \delta s_{1}\right)\left(\mathbf{t}_{\mathbf{1}}+\frac{\partial \mathbf{t}_{\mathbf{1}}}{\partial s_{1}} \delta s_{1}\right)(h)(1)-\left(T_{1} \mathbf{t}_{\mathbf{1}}\right)(h)(1)
$$

where $T_{1}$ is the membrane stress and $\mathbf{t}_{\mathbf{1}}$ is the unit tangent vector along the main membrane direction $s_{1}$. 


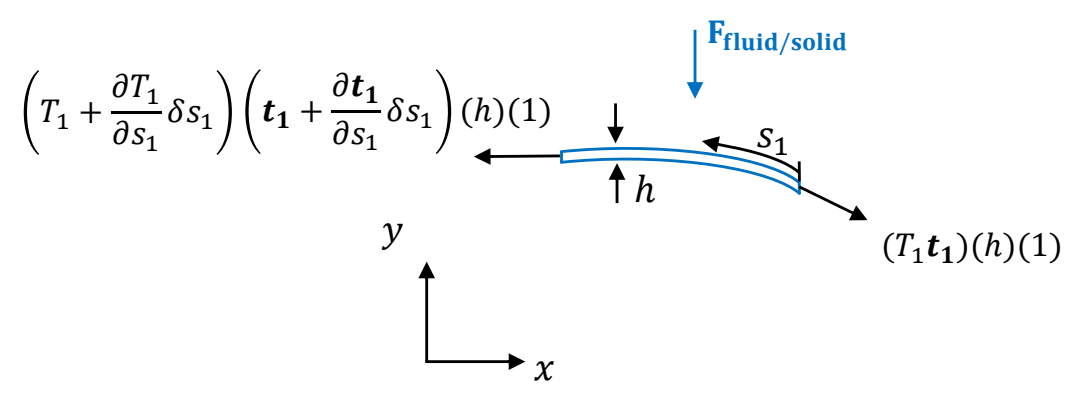

Figure 3.3: Free-body diagram of the 1-D membrane element (gravity force neglected).

Considering a massless membrane element and that the second-order terms $\left(\delta s_{1}\right)^{2}$ are neglected, the net force, which has tangential and normal components to the $s_{1}$-direction, balances the force exerted on the solid $\mathbf{F}_{\text {fluid/solid }}$ as follows:

$$
\frac{\partial\left(T_{1} \mathbf{t}_{\mathbf{1}}\right)}{\partial s_{1}} \delta s_{1} h+\mathbf{F}_{\text {fluid } / \text { solid }}=\mathbf{0}
$$

recalling that $\mathbf{F}_{\text {fluid/solid }}=-\mathbf{F}_{\text {solid/fluid }}$ and dividing eq. (3-6) by $\delta V=\delta s_{1} \times h \times 1$, it is concluded that the force per unit volume $\mathbf{f}$, i.e. force density that the membrane exerts on the fluid [42], is

$$
\mathbf{f}=\frac{\partial\left(T_{1} \mathbf{t}_{\mathbf{1}}\right)}{\partial s_{1}}
$$

Furthermore, considering that in the membrane element, the stress normal to the surface $T_{3}$ is small when compared to other stresses, that there is no deformation along the $z$-direction $\left(\varepsilon_{2}=0\right)$ because the membrane is infinite along the $s_{2}$-direction, and the membrane material obeys Hooke's law, $T_{1}$ depends on the strain $\varepsilon_{1}$ (see Appendix A.1):

$$
T_{1}=\left(\frac{E}{1-\nu^{2}}\right) \varepsilon_{1},
$$

where $E$ and $\nu$ are Young's modulus and Poisson's ratio of the membrane material, respectively. The strain $\varepsilon_{1}$ is defined as the ratio of the deformation 
at any time $t$ to the unstressed arc-length $s_{1 \infty}$ of a given membrane element, and calculated for an unstressed reference configuration as $\varepsilon_{1}=\left|\frac{\partial \mathbf{X}}{\partial s_{1 \infty}}\right|-1$, as discussed by Peskin \& McQueen [42].

After rearranging all terms, and defining that at $t=0$, the capsule is in a prestressed state with tension $T_{0}$ [43], $T_{1}$ can be written as

$$
T_{1}\left(\mathbf{X}\left(s_{1}, t\right)\right)=\left(\frac{E}{1-\nu^{2}}\right)\left(\frac{s_{1}(t)-s_{1}(0)}{s_{1 \infty}}\right)+T_{0},
$$

where $s_{1}(t), s_{1}(0)$, and $s_{1 \infty}$ are the arc-length of the membrane element at any time $t$, time $t=0$, and at the unstressed state, respectively.

The prestressed tension $T_{0}$, which defines the initial pressure difference between inner and outer phases, is expressed as

$$
T_{0}=\alpha\left(\frac{E}{1-\nu^{2}}\right)
$$

where $\alpha$ is the preinflation parameter, defined as $\alpha=\frac{s_{1}(0)}{s_{1 \infty}}-1$. It can be also determined in terms of prestressed and unstressed capsule radii $a$ and $a_{0}$ [44], respectively:

$$
\alpha=\frac{a}{a_{0}}-1
$$

\subsection{2}

\section{The axisymmetric flow}

In the axisymmetric flow, the membrane is generated by the rotation of a plane curve, named meridian, about the $x$-axis. Any point of the membrane is determined by the specific meridian and by a second coordinate, known as parallel, which varies along the meridian and is constant on a circle, defined as parallel circle, around the membrane axis [41, 43]. Meridian and parallel are identified by angles $\phi$ and $\theta$, respectively. 
Any membrane element is defined by two meridians and two parallel circles. Figure 3.4 shows all normal stresses that act on the membrane element. In this study, the membrane element projected onto its tangent plane is approximated as a rectangular region. Equilibrium conditions can provide the expressions for $T_{1}$ and $T_{2}$, in order to obtain $\mathbf{f}$ for an axisymmetric configuration.

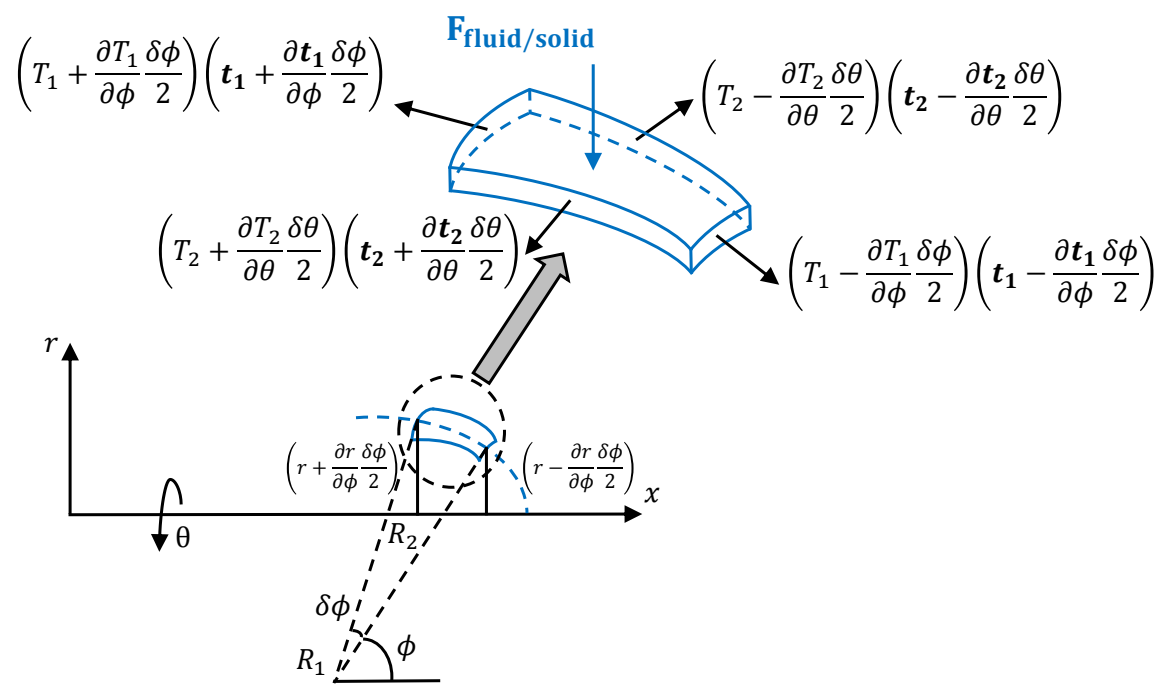

Figure 3.4: Normal stresses acting on the membrane element. Forces exerted on the membrane also included.

Thus, the net force along the $\phi$-direction, i.e. parallel to the tangent to the meridian, is written as

$$
\begin{aligned}
\left(T_{1}+\frac{\partial T_{1}}{\partial \phi} \frac{\delta \phi}{2}\right)\left(\mathbf{t}_{\mathbf{1}}\right. & \left.+\frac{\partial \mathbf{t}_{\mathbf{1}}}{\partial \phi} \frac{\delta \phi}{2}\right)\left(r+\frac{\partial r}{\partial \phi} \frac{\delta \phi}{2}\right) \delta \theta h \\
& -\left(T_{1}-\frac{\partial T_{1}}{\partial \phi} \frac{\delta \phi}{2}\right)\left(\mathbf{t}_{\mathbf{1}}-\frac{\partial \mathbf{t}_{\mathbf{1}}}{\partial \phi} \frac{\delta \phi}{2}\right)\left(r-\frac{\partial r}{\partial \phi} \frac{\delta \phi}{2}\right) \delta \theta h
\end{aligned}
$$

Notice that $\frac{\partial r}{\partial \phi}$ term is included because the length of section $r \delta \theta$ changes with $\phi$. Considering that second- and third-order terms, i.e. $(\delta \phi)^{2}$ and $(\delta \phi)^{3}$, are neglected, the following expression is obtained:

$$
\left(T_{1} \mathbf{t}_{\mathbf{1}} \frac{\partial r}{\partial \phi} \delta \phi+T_{1} \frac{\partial \mathbf{t}_{\mathbf{1}}}{\partial \phi} r \delta \phi+\frac{\partial T_{1}}{\partial \phi} \delta \phi \mathbf{t}_{\mathbf{1}} r\right) \delta \theta h
$$


which yields

$$
\frac{\partial\left(r T_{1} \mathbf{t}_{\mathbf{1}}\right)}{\partial \phi} \delta \phi \delta \theta h
$$

In a similar manner, the net force in the $\theta$-direction, acting on surface area $R_{1} \delta \phi h$, can be expressed as

$$
\left(T_{2}+\frac{\partial T_{2}}{\partial \theta} \frac{\delta \theta}{2}\right)\left(\mathbf{t}_{\mathbf{2}}+\frac{\partial \mathbf{t}_{\mathbf{2}}}{\partial \theta} \frac{\delta \theta}{2}\right) R_{1} \delta \phi h-\left(T_{2}-\frac{\partial T_{2}}{\partial \theta} \frac{\delta \theta}{2}\right)\left(\mathbf{t}_{\mathbf{2}}-\frac{\partial \mathbf{t}_{\mathbf{2}}}{\partial \theta} \frac{\delta \theta}{2}\right) R_{1} \delta \phi h
$$

where $R_{1}$ is the radius of curvature which describes the local arc-length of the surface meridian, as defined by Evans \& Skalak [43]. After neglecting second-order terms $(\delta \theta)^{2}$, the net force in the $\theta$-direction is written as

$$
\frac{\partial\left(T_{2} \mathbf{t}_{\mathbf{2}}\right)}{\partial \theta} \delta \theta R_{1} \delta \phi h
$$

Considering a massless membrane element, and that net forces acting along $\phi$ and $\theta$ directions, eqs. (3-14) and (3-16) are in equilibrium with the force the fluid exerts on the solid $\mathbf{F}_{\text {fluid/solid, }}$ the equilibrium equation is written as

$$
\frac{\partial\left(r T_{1} \mathbf{t}_{\mathbf{1}}\right)}{\partial \phi} \delta \phi \delta \theta h+\frac{\partial\left(T_{2} \mathbf{t}_{\mathbf{2}}\right)}{\partial \theta} \delta \theta R_{1} \delta \phi h+\mathbf{F}_{\text {fluid } / \text { solid }}=\mathbf{0}
$$

Recalling that $\mathbf{F}_{\text {fluid/solid }}=-\mathbf{F}_{\text {solid/fluid }}, d s_{1}=R_{1} d \phi$, and $d s_{2}=r d \theta$; and dividing eq. (3-17) by $\delta V=\delta s_{1} \delta s_{2} h=R_{1} \delta \phi r \delta \theta h$, the force per unit volume $\mathbf{f}$ is finally obtained as

$$
\mathbf{f}=\frac{1}{r} \frac{\partial\left(r T_{1} \mathbf{t}_{\mathbf{1}}\right)}{\partial s_{1}}+\frac{\partial\left(T_{2} \mathbf{t}_{\mathbf{2}}\right)}{\partial s_{2}} .
$$


Notice that, the coordinates of the Lagrangian points of the membrane $\mathbf{X}$ are defined as $\mathbf{X}=\mathbf{X}\left(s_{1}, s_{2}, t\right)$, where $s_{1} \in\left[0, l_{s}(t)\right]$ is the coordinate along the meridian and $s_{2} \in[0,2 \pi r]$ is the coordinate along the parallel circle.

Considering that the membrane material obeys Hooke's law (HK), which is a simple stress-strain relation, $T_{1}$ and $T_{2}$ are expressed as

$$
\left(\begin{array}{l}
T_{1} \\
T_{2}
\end{array}\right)=\frac{E}{1-\nu^{2}}\left(\begin{array}{ll}
1 & \nu \\
\nu & 1
\end{array}\right)\left(\begin{array}{l}
\varepsilon_{\phi} \\
\varepsilon_{\theta}
\end{array}\right)+\left(\begin{array}{l}
T_{0} \\
T_{0}
\end{array}\right)
$$

where the meridional strain is defined as $\varepsilon_{\phi}=\frac{1}{2}\left(\lambda_{1}^{2}-1\right)$, and the azimuthal strain is defined as $\varepsilon_{\theta}=\frac{1}{2}\left(\lambda_{2}^{2}-1\right)$, with $\lambda_{1}=\frac{\partial s_{1}}{\partial s_{1}}$ and $\lambda_{2}=\frac{r}{r_{0}}$. Both, $\lambda_{1}$ and $\lambda_{2}$, depend on coordinates $(x, r)$ and $\left(x_{0}, r_{0}\right)$ for the instantaneous and initial states, respectively, as discussed by Evans \& Skalak [43]. Furthermore, the initial prestress $T_{0}$ depends on initial $\lambda_{1}=\lambda_{2}=\lambda_{0}=1+\alpha$, as studied by Lefebvre \& Barthès-Biesel [44].

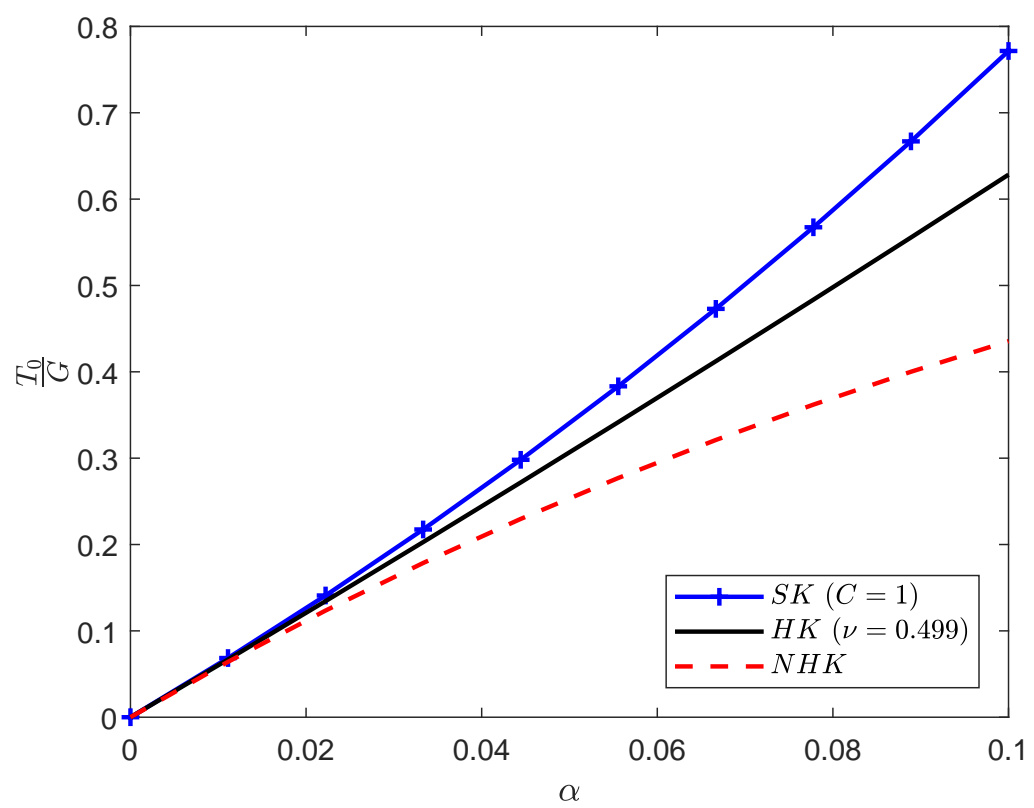

Figure 3.5: Initial prestress $T_{0}$ as a function of preinflation parameter $\alpha$ for SK, HK, and NHK membrane constitutive equations.

Figure 3.5 illustrates how the initial prestress $T_{0}$ and the preinflation parameter $\alpha$ relate to each other for two additional membrane constitutive 
equations [45], which are described as follows:

- Skalak's model (SK), which exhibits a strain-hardening response, was originally used to model red blood cell membranes [46]:

$$
T_{1}=\frac{G}{\lambda_{1} \lambda_{2}}\left[\lambda_{1}^{2}\left(\lambda_{1}^{2}-1\right)+C\left(\lambda_{1} \lambda_{2}\right)^{2}\left[\left(\lambda_{1} \lambda_{2}\right)^{2}-1\right]\right]
$$

where $G=G_{s} / h$ is the material shear modulus and $C$ is the dimensionless parameter that measures the ratio of area dilation modulus to surface shear modulus $G_{s}$, and considered here as $C=1$ [44].

- Neo-Hookean model (NHK), which presents a strain-softening response, is utilized for infinitively thin sheet, and expressed as [47]

$$
T_{1}=\frac{G}{\lambda_{1} \lambda_{2}}\left[\lambda_{1}^{2}-\frac{1}{\left(\lambda_{1} \lambda_{2}\right)^{2}}\right]
$$

For eqs. (3-20) and (3-21), $\lambda_{1}$ and $\lambda_{2}$ are calculated as described for Hooke's law. And, $T_{2}$ is defined by interchanging indices 1 and 2 .

In both problems, the 2-D planar flow and the axisymmetric flow, the capsule membrane is advected by the velocity field considering no-slip condition:

$$
\begin{aligned}
\frac{\partial \mathbf{X}}{\partial t} & =\int_{\Omega_{f}} \mathbf{u} \delta_{D}(\mathbf{x}-\mathbf{X}) d \Omega_{f}^{\prime} \\
& =\mathbf{u}(\mathbf{X}, t)
\end{aligned}
$$




\section{3}

\section{Boundary and initial conditions}

In order to fully define the problem, boundary and initial conditions are required. The boundary conditions used are summarized in Fig. 3.6.

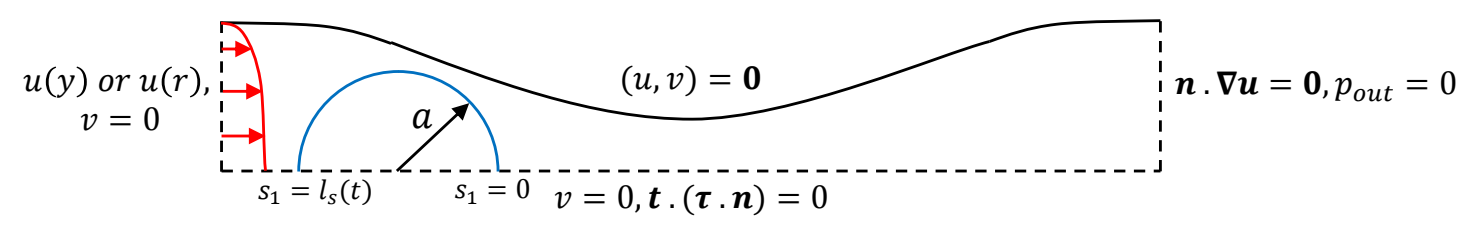

Figure 3.6: Boundary conditions for channel (or capillary) geometry.

For the 2-D planar flow, at the inlet plane, a parabolic velocity profile is imposed:

$$
u(x=0, y)=\frac{3 \bar{V}}{2}\left[1-\left(\frac{y}{H_{s}}\right)^{2}\right], v(x=0, y)=0
$$

where $\bar{V}$ is the average velocity of the continuous phase and $H_{s}$ is the half the channel height at the inlet.

For the axisymmetric flow, at the inlet plane, the imposed velocity profile becomes

$$
u(x=0, r)=2 \bar{V}\left[1-\left(\frac{r}{R_{s}}\right)^{2}\right], v(x=0, r)=0
$$

where $R_{s}$ is the inlet capillary radius.

Leyrat-Maurin \& Barthès-Biesel [32] studied an alternative boundary condition at which the inlet pressure is constant and imposed, leading to a varying flow rate. In such circumstances, if the imposed inlet pressure is not high enough, the flow could be completely blocked. In the present study, however, the inlet pressure varies as the capsule flows through the constricted geometry in order to ensure that the flow rate remains constant. For that reason, the current model is not able to predict full blockage of the channel or capillary. 
For both problems, at the outflow plane, a fully developed flow and a fixed outflow pressure are imposed:

$$
\left.\mathbf{n} \cdot \nabla \mathbf{u}\right|_{x=L}=\mathbf{0}, p_{\text {out }}=0
$$

where $\mathbf{n}$ is the unit vector normal to the outflow boundary. Along the wall, no-slip and no-penetration conditions are imposed:

$$
\mathbf{u}=\mathbf{0}
$$

Along the symmetry plane (or symmetry axis), vanishing vertical (or radial) velocity, and zero tangential stress are considered:

$$
v=0, \mathbf{t} \cdot(\boldsymbol{\tau} \cdot \mathbf{n})=0
$$

where $\mathbf{t}$ and $\mathbf{n}$ are the unit tangent vector and unit normal vector at the symmetry plane (or axis), respectively, and $\boldsymbol{\tau}$ is the stress tensor at the symmetry boundary.

In order to ensure symmetry of the capsule, a geometric constraint is imposed on the extreme points of the solid domain, at $s_{1}=0$ and $s_{1}=l_{s}(t)$ :

$$
\left.\frac{\partial X}{\partial s_{1}}\right|_{s_{1}=0, l_{s}(t)}=0
$$

where $\mathbf{X}=(X, Y)$ defines the capsule membrane and $l_{s}(t)$ is the solid domain length at any time $t$.

The axisymmetric flow, requires one additional geometric condition:

$$
\left.\frac{\partial^{2} Y}{\partial s_{1}^{2}}\right|_{s_{1}=0, l_{s}(t)}=0
$$


this condition is related to the second radius of curvature $R_{2} . R_{2}$ is measured on a normal to the meridian between its intersection with the surface and the $x$-axis (see Fig. 3.4), as defined by Flügge [41]. At both capsule ends, i.e. $s_{1}=0$ and $s_{1}=l_{s}(t)$, all meridians meet, and eq. (3-29) guarantees that the vector normal to the membrane surface is perfectly horizontal, i.e. $\frac{\partial \mathbf{t}_{1}}{\partial s_{1}}$ is parallel to the $x$-axis, leading to a continuous and smooth variation of the principal radii of curvature [41].

Finally, at $t=0$, zero velocity and pressure fields are defined. The center of gravity of the capsule is known and the prestressed condition is defined by the preinflation parameter $\alpha$. 


\section{4}

\section{Numerical solution}

This chapter focuses on the main features of the finite element method, and a detailed analysis of the coupling of solid and liquid phases using the immersed boundary method. Further details of the finite element method can be found in specialized references, including Carvalho \& Valério [48].

The system of partial differential equations which describes the flow of the liquid phase and capsule deformation was solved by the method of weighted residuals using finite element basis functions.

\section{1}

\section{Finite element and immersed boundary methods}

The weak formulation is obtained by multiplying the momentum and mass conservation equations (see eqs. (3-2)) with the appropriate vector and scalar weighting functions $(\mathbf{W}, \chi)$, integrating over the entire fluid domain $\Omega_{f}$, and applying the divergence theorem to the second-order terms. Galerkin's method was applied to both differential equations, i.e. the weighting functions are equal to the corresponding basis functions.

The weighted residual equations are written as

$$
\begin{aligned}
R_{m} & =\int_{\Omega_{f}}\left[\rho\left(\frac{\partial \mathbf{u}}{\partial t}+\mathbf{u} \cdot \nabla \mathbf{u}\right)-\nabla \cdot \boldsymbol{\tau}-\mathbf{f}^{\mathbf{B}}\right] \cdot \mathbf{W} d \Omega_{f}^{\prime}, \\
R_{m c} & =\int_{\Omega_{f}}(\boldsymbol{\nabla} \cdot \mathbf{u}) \chi d \Omega_{f}^{\prime} .
\end{aligned}
$$

In their book, Carvalho \& Valério [48] discussed in detail most of the terms in eqs. (4-1), except for the last term of the momentum equation $\mathbf{f}^{\mathbf{B}}$, which receives special attention in this work. Thus, the term $R_{m}^{\mathrm{f}^{\mathrm{B}}}$, responsible for coupling fluid flow and membrane dynamics, is expressed as 


$$
R_{m}^{\mathbf{f}^{\mathrm{B}}}=-\int_{\Omega_{f}} \mathbf{f}^{\mathbf{B}} \cdot \mathbf{W} d \Omega_{f}^{\prime}
$$

substituting eq. (3-4) into eq. (4-2) yields

$$
\begin{aligned}
R_{m}^{\mathrm{f}^{\mathrm{B}}} & =-\int_{\Omega_{f}} \int_{\Omega_{s}} \mathbf{f} \delta_{D}(\mathbf{x}-\mathbf{X}) d \Omega_{s}^{\prime} \cdot \mathbf{W} d \Omega_{f}^{\prime} \\
& =-\int_{\Omega_{s}} \mathbf{f} \cdot \mathbf{W}(\mathbf{X}) d \Omega_{s}^{\prime}
\end{aligned}
$$

Notice that, the coupling between solid and fluid phases requires evaluating the weighting function $\mathbf{W}$ at the membrane position $\mathbf{X}$.

The fluid domain was divided into quadrilateral elements. Velocity and pressure fields were approximated with a linear combination of a finite number of basis functions, and expressed as

$$
\begin{aligned}
& \mathbf{u}=\left(\begin{array}{l}
u \\
v
\end{array}\right)=\left(\begin{array}{l}
\sum_{j=1}^{9} U_{j}(t) \phi_{j}^{u} \\
\sum_{j=1}^{9} V_{j}(t) \phi_{j}^{u}
\end{array}\right), \\
& p=\sum_{j=1}^{3} P_{j}(t) \chi_{j}^{p} .
\end{aligned}
$$

The basis functions used were Lagrangian biquadratic polynomial for velocity $\phi^{u}$ and linear discontinuous for pressure $\chi^{p} . U_{j}(t), V_{j}(t)$, and $P_{j}(t)$ are the basis functions coefficients and the unknowns of the problem.

Natural boundary conditions are applied by computing boundary integrals, as a straightforward consequence of the divergence theorem. The essential boundary conditions are imposed by replacing the corresponding residual with a desired velocity value. 


\section{2}

\section{Detailed formulation of $R_{m}^{\mathrm{f}^{\mathrm{B}}}$}

As already discussed, the term $R_{m}^{\mathbf{f}^{\mathrm{B}}}$ is an additional term in the momentum residual that accounts for fluid-solid coupling, with its general form expressed in eq. (4-3).

\subsection{1}

\section{The 2-D planar flow}

For the 2-D planar flow, by combining eqs. (3-7) and (4-3), and considering that, for a 1-D membrane model, $d \Omega_{s}^{\prime}=d s_{1} \times h \times 1$, the following expression is obtained:

$$
R_{m}^{\mathbf{f}^{\mathrm{B}}}=-\int_{0}^{l_{s}(t)} \frac{\partial\left(T_{1} \mathbf{t}_{\mathbf{1}}\right)}{\partial s_{1}} \cdot \mathbf{W}(\mathbf{X}) h d s_{1}
$$

integration by parts leads to

$$
R_{m}^{\mathrm{f}^{\mathrm{B}}}=-\left.T_{1} \mathbf{t}_{\mathbf{1}} \cdot \mathbf{W}(\mathbf{X}) h\right|_{0} ^{l_{s}(t)}+\int_{0}^{l_{s}(t)} T_{1} \mathbf{t}_{\mathbf{1}} \cdot h d \mathbf{W}(\mathbf{X})
$$

Observe that, the first term of eq. (4-6) only contributes to the vertical momentum equation residual. For that reason, this term is trivial since the momentum equation in the $y$-direction is substituted by the essential boundary condition $v=0$ along the symmetry plane. Therefore, eq. (4-6) becomes only the line integral along the capsule membrane expressed as

$$
R_{m}^{\mathbf{f}^{\mathrm{B}}}=\int_{0}^{l_{s}(t)} T_{1} h \mathbf{t}_{\mathbf{1}} \cdot d \mathbf{W}(\mathbf{X})
$$

recalling that $\mathbf{W}$, defined by the weighting functions $\boldsymbol{\psi}_{\boldsymbol{i}}$ is equal to $\boldsymbol{\phi}_{\boldsymbol{i}}$, because Galerkin's method is used [48].

The membrane is divided into 1-D linear segments and the line integral is evaluated using this 1-D mesh as follows: 


$$
R_{m i}^{\mathbf{f}^{\mathrm{B}}}=\sum_{e=1}^{n_{L}-1} T_{1}(e) h \mathbf{t}_{\mathbf{1}}(e) \cdot\left[\boldsymbol{\phi}_{i}\left(\mathbf{X}_{e+1}\right)-\boldsymbol{\phi}_{i}\left(\mathbf{X}_{e}\right)\right]
$$

where $e$ is a $1-\mathrm{D}$ membrane segment and $n_{L}$ is the number of Lagrangian points in the discretized solid domain.

It is worth noting that $\boldsymbol{\phi}_{i}\left(\mathbf{X}_{k_{n}}\right), k_{n}=e, e+1$ is non-null only if the solid domain node $k_{n}$ is inside a 2-D element $f_{e}$ at which $\phi_{i}$ is non-null. Thus, the sum (see eq. (4-8)) is only evaluated along the linear segments at which $\phi_{i}$ is non-null, as illustrated in Fig. 4.1.

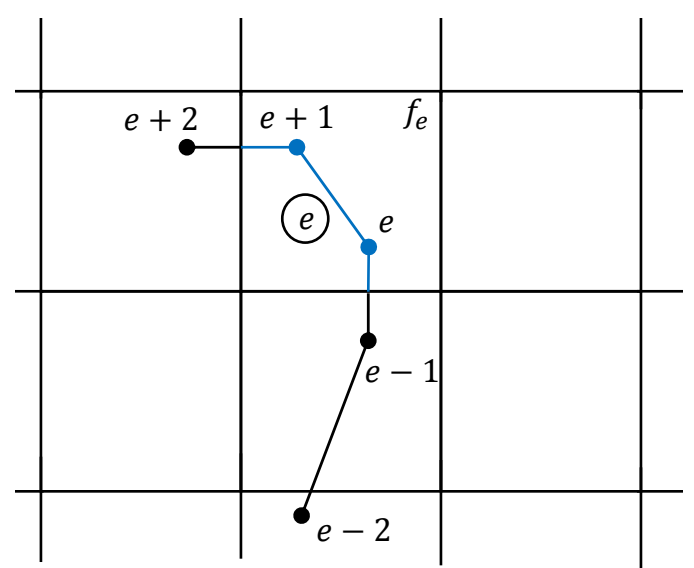

Figure 4.1: Discretization of the membrane and fluid domains.

In order to evaluate $\boldsymbol{\phi}_{i}\left(\mathbf{X}_{k_{n}}\right)$, it is necessary to map the global coordinates of node $k_{n}$ to the local coordinate system of the 2-D fluid element, at which $\phi_{i}$ is defined.

For that reason, a quadratic inverse mapping is used in this work. The quadratic inverse mapping requires to find $(\xi, \eta)$ for a given $(x, y)$, as sketched in Fig. (4.2).

To do so, a second-order Taylor polynomial of $(x, y)$ about the point $\left(x_{9}, y_{9}\right)$, center of the Eulerian element, is obtained as 


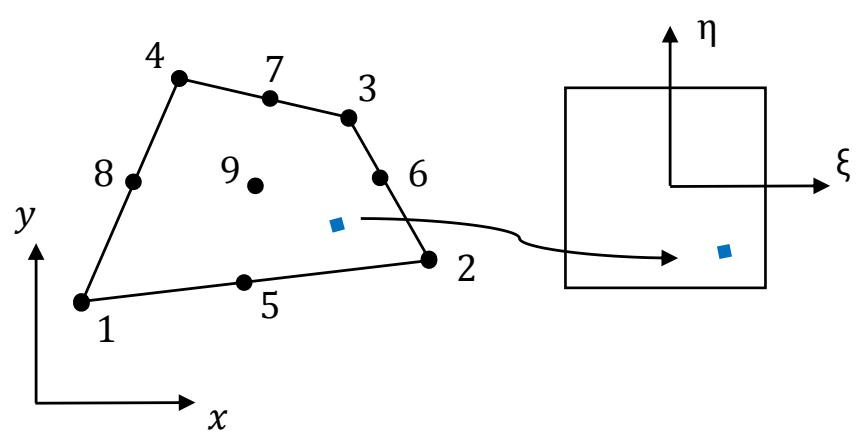

Figure 4.2: Given a global position, find the local coordinates.

$$
\begin{aligned}
x-x_{9}= & \left.\frac{\partial x}{\partial \xi}\right|_{0} \Delta \xi+\left.\frac{\partial x}{\partial \eta}\right|_{0} \Delta \eta+\frac{1}{2}\left[\left.\frac{\partial^{2} x}{\partial \xi^{2}}\right|_{0} \Delta \xi^{2}\right. \\
& \left.+\left.\frac{\partial^{2} x}{\partial \eta^{2}}\right|_{0} \Delta \eta^{2}+\left.2 \frac{\partial^{2} x}{\partial \eta \partial \xi}\right|_{0} \Delta \eta \Delta \xi\right], \\
y-y_{9}= & \left.\frac{\partial y}{\partial \xi}\right|_{0} \Delta \xi+\left.\frac{\partial y}{\partial \eta}\right|_{0} \Delta \eta+\frac{1}{2}\left[\left.\frac{\partial^{2} y}{\partial \xi^{2}}\right|_{0} \Delta \xi^{2}\right. \\
& \left.+\left.\frac{\partial^{2} y}{\partial \eta^{2}}\right|_{0} \Delta \eta^{2}+\left.2 \frac{\partial^{2} y}{\partial \eta \partial \xi}\right|_{0} \Delta \eta \Delta \xi\right]
\end{aligned}
$$

where $\Delta \xi=\xi-0$ and $\Delta \eta=\eta-0$.

Recalling that $x=x(\xi, \eta), y=y(\xi, \eta)$, and their respective first and second derivatives are known, then a multivariable nonlinear system is solved iteratively by using the Newton-Raphson method.

\subsection{2}

\section{The axisymmetric flow}

Solving the axisymmetric problem requires the combination of eqs. (3-18) and (4-3), and to consider a 2-D membrane model, $d \Omega_{s}^{\prime}=d s_{1} \times d s_{2} \times h$, with $d s_{2}=r d \theta$.

Firstly, integrating with respect to $\theta$, from 0 to $1 \mathrm{rad}$, leads to 


$$
\begin{aligned}
R_{m}^{\mathbf{f}^{\mathbf{B}}=} & -\int_{0}^{1} \int_{0}^{l_{s}(t)}\left[\frac{1}{r} \frac{\partial\left(r T_{1} \mathbf{t}_{\mathbf{1}}\right)}{\partial s_{1}}+\frac{\partial\left(T_{2} \mathbf{t}_{\mathbf{2}}\right)}{\partial s_{2}}\right] \cdot \mathbf{W}(\mathbf{X}) h d s_{1}(r d \theta) \\
= & -\int_{0}^{1} \int_{0}^{l_{s}(t)} \frac{1}{r} \frac{\partial\left(r T_{1} \mathbf{t}_{\mathbf{1}}\right)}{\partial s_{1}} \cdot \mathbf{W}(\mathbf{X}) h r d s_{1} d \theta \\
& -\int_{0}^{1} \int_{0}^{l_{s}(t)} \frac{\partial\left(T_{2} \mathbf{t}_{\mathbf{2}}\right)}{\partial s_{2}} \cdot \mathbf{W}(\mathbf{X}) h r d s_{1} d \theta
\end{aligned}
$$

which reduces to

$$
R_{m}^{\mathbf{f}^{\mathbf{B}}}=-\int_{0}^{l_{s}(t)} \frac{1}{r} \frac{\partial\left(r T_{1} \mathbf{t}_{\mathbf{1}}\right)}{\partial s_{1}} \cdot \mathbf{W}(\mathbf{X}) h r d s_{1}-\int_{0}^{l_{s}(t)} \frac{\partial\left(T_{2} \mathbf{t}_{\mathbf{2}}\right)}{\partial s_{2}} \cdot \mathbf{W}(\mathbf{X}) h r d s_{1}
$$

integrating by parts the first term and using the product rule for derivatives in the second term of eq. (4-11) yield

$$
\begin{aligned}
R_{m}^{\mathbf{f}^{\mathbf{B}}=} & -\left.r T_{1} \mathbf{t}_{\mathbf{1}} \cdot \mathbf{W}(\mathbf{X}) h\right|_{0} ^{l_{s}(t)}+\int_{0}^{l_{s}(t)} T_{1} \mathbf{t}_{\mathbf{1}} r h \cdot d \mathbf{W}(\mathbf{X}) \\
& -\int_{0}^{l_{s}(t)}\left[\frac{\partial T_{2}}{\partial s_{2}} \mathbf{t}_{\mathbf{2}}+T_{2} \frac{\partial \mathbf{t}_{\mathbf{2}}}{\partial s_{2}}\right] \cdot \mathbf{W}(\mathbf{X}) h r d s_{1}
\end{aligned}
$$

Notice that, the first term is identically zero, because $r=0$ at $s_{1}=0$ and $s_{1}=l_{s}(t)$ (at the symmetry axis). Furthermore, $\frac{\partial T_{2}}{\partial s_{2}}=\frac{\partial T_{2}}{r \partial \theta}=0$ due to axisymmetry; therefore, the expression is reduced to

$$
R_{m}^{\mathbf{f}^{\mathrm{B}}}=\int_{0}^{l_{s}(t)} T_{1} \mathbf{t}_{\mathbf{1}} r h \cdot d \mathbf{W}(\mathbf{X})-\int_{0}^{l_{s}(t)} T_{2} \frac{\partial \mathbf{t}_{\mathbf{2}}}{\partial s_{2}} \cdot \mathbf{W}(\mathbf{X}) h r d s_{1}
$$

recalling that $\frac{\partial \mathbf{t}_{\mathbf{2}}}{\partial s_{2}}=\frac{\partial \mathbf{t}_{\mathbf{2}}}{r \partial \theta}=-\frac{\mathbf{e}_{\mathbf{r}}}{r}$, then eq. (4-13) can be further simplified to

$$
R_{m}^{\mathbf{f}^{\mathrm{B}}}=\int_{0}^{l_{s}(t)} T_{1} r h \mathbf{t}_{\mathbf{1}} \cdot d \mathbf{W}(\mathbf{X})+\int_{0}^{l_{s}(t)} T_{2} h \mathbf{e}_{\mathbf{r}} \cdot \mathbf{W}(\mathbf{X}) d s_{1}
$$


Similar to the 2-D planar case, the line integral along the solid domain can be computed as

$$
\begin{aligned}
R_{m i}^{\mathbf{f}^{\mathrm{B}}}= & \sum_{e=1}^{n_{L}-1} T_{1}(e) r(e) h \mathbf{t}_{\mathbf{1}}(e) \cdot\left[\boldsymbol{\phi}_{i}\left(\mathbf{X}_{e+1}\right)-\boldsymbol{\phi}_{i}\left(\mathbf{X}_{e}\right)\right] \\
& +\sum_{e=1}^{n_{L}-1} T_{2}(e) h \mathbf{e}_{\mathbf{r}} \cdot \boldsymbol{\phi}_{i}\left(\mathbf{X}_{e}\right)\left|\mathbf{X}_{e+1}-\mathbf{X}_{e}\right|
\end{aligned}
$$

where $\mathbf{t}_{\mathbf{1}}(e)$ is the unit tangent vector of the $e$-membrane segment and $\mathbf{e}_{\mathbf{r}}$ is the unit radial vector defined as $(0,1)$, as sketched in Fig. 4.3.

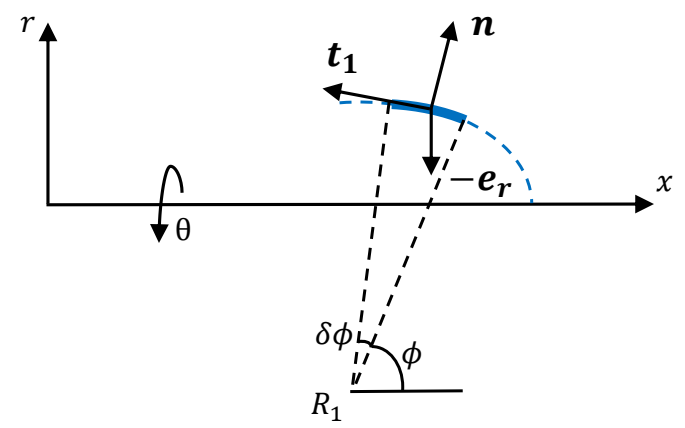

Figure 4.3: $\mathbf{t}_{\mathbf{1}}, \mathbf{n}$, and $\mathbf{e}_{\mathbf{r}}$ are unit tangent vector, unit normal vector, and unit radial vector, respectively.

As mentioned in the previous chapter, a rectangular region is an approximation of the projected membrane surface onto its tangent plane. A different approximation would be a trapezoidal region, which would include additional force components. In order to reduce the difference between both approximations (regarding force components), this study requires a refined membrane grid.

For both flows (2-D planar and axisymmetric), after obtaining all terms of the momentum and mass conservation equation residuals [48], including the fluid-solid coupling term $R_{m i}^{\mathrm{f}^{\mathrm{B}}}$, and after replacing each field by the corresponding expansion in terms of the basis functions, the partial differential equations that describe the fluid flow become a set of nonlinear algebraic equations defined by $\mathbf{R}=\left[R_{m i}, R_{m c i}\right]^{T}=\mathbf{0}$, with a time-dependent solution vector $\mathbf{S v}=\left[U_{j}(t), V_{j}(t), P_{j}(t)\right]^{T}$, where $i$ and $j$ are the indices of the residual 
and solution vectors, respectively.

\section{3}

\section{Solution of the transient problem}

Time discretization utilized a predictor-corrector algorithm, in which the predictor step is the backward Euler method. The initial guess of the next time step $\mathbf{S v}_{k_{t}+1}^{(0)}$ is obtained as a linear extrapolation using the two previous solution vectors.

$$
\mathbf{S v}_{k_{t}+1}^{(0)}=\mathbf{S} \mathbf{v}_{k_{t}}+\frac{\mathbf{S v}_{k_{t}}-\mathbf{S v}_{k_{t}-1}}{t_{k_{t}}-t_{k_{t}-1}}\left(t_{k_{t}+1}-t_{k_{t}}\right)
$$

where $k_{t}+1, k_{t}$, and $k_{t}-1$ are the next, current, and previous time steps, respectively. The corrector step is a first-order implicit Euler method, further detailed in the next section.

\section{4}

\section{Solution of the nonlinear problem}

The set of nonlinear algebraic equations is solved by the NewtonRaphson method, which with the appropriate initial guess shows a quadratic convergence. Thus, the following system needs to be solved iteratively until the criterion $\|\mathbf{R}\| \leq$ error is satisfied:

$$
\begin{aligned}
\Delta \mathbf{S} \mathbf{v}^{\left(k_{i}+1\right)} & =-\left(\frac{1}{\Delta t} \mathbf{M}\left(\mathbf{S} \mathbf{v}_{k_{t}+1}^{\left(k_{i}\right)}\right)+\mathbf{J}\left(\mathbf{S v}_{k_{t}+1}^{\left(k_{i}\right)}\right)\right)^{-1} \mathbf{R}\left(\mathbf{S v}_{k_{t}+1}^{\left(k_{i}\right)}, \mathbf{S v}_{k_{t}}\right), \\
\mathbf{S v}_{k_{t}+1}^{\left(k_{i}+1\right)} & =\mathbf{S v}_{k_{t}+1}^{\left(k_{i}\right)}+\Delta \mathbf{S} \mathbf{v}^{\left(k_{i}+1\right)},
\end{aligned}
$$

where $k_{i}+1$ and $k_{i}$ are the current and previous Newton-Raphson iteration indices, respectively. This method implies the calculation of the Jacobian matrix $\mathbf{J}=\frac{\partial \mathbf{R}}{\partial \mathbf{S v}}$, which measures the sensitivity of the residuals to the problem unknowns, and the mass matrix $\mathbf{M}=\frac{\partial \mathbf{R}}{\partial \dot{\mathbf{S i v}}}$, which measures the sensitivity of the residuals to the time derivatives of the unknowns [49]. At each iteration, the linear system is solved using the MATLAB ${ }^{\circledR}$ direct solver [50]. 


\section{5}

\section{Update of the membrane position $\mathrm{X}$}

As time elapses, the membrane moves and its position requires to be updated at each time step. Equation (3-22) shows that it is necessary to interpolate velocity $\mathbf{u}$ at the membrane position $\mathbf{X}$. The already explained quadratic inverse mapping is used. Afterwards, the next membrane position $\mathbf{X}$ is explicitly updated by the fourth-order Runge-Kutta method.

Eventually, it is possible that a solid element $e$ crosses a 2-D fluid element $f_{e}$ without having a solid node located inside the fluid element, as shown in Fig. 4.4. It is important that the segment of the 1-D mesh that is inside the element $f_{e}$ is computed in the residual equations associated with the weighting functions defined in the element $f_{e}$. For this reason, an extra solid node is created in the middle of the $a b$-segment in order to compute the contribution of this segment to the momentum residual.

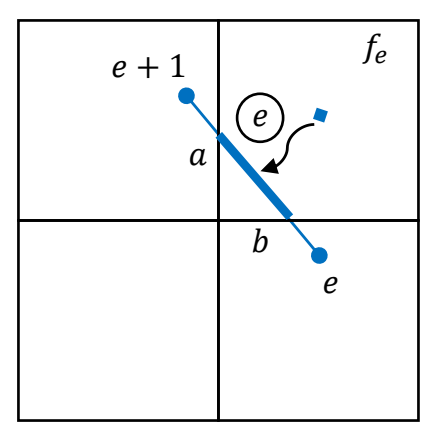

Figure 4.4: An extra node is created to compute the line integral.

\section{6}

\section{Time-stepping scheme}

Regarding the numerical time integration, it should be pointed out that the momentum weighted residual at a given time step depends on the current configuration of the capsule membrane, through the body force term $\mathbf{f}^{\mathbf{B}}$ and the definition of the membrane stress. The system of equations is decoupled at each time step using an implicit time integration for the momentum equation and the mass conservation equation, and an explicit time integration to update the position of the membrane mesh [51]. Velocity and pressure fields $\mathbf{u}_{k_{t}+1}$ and $p_{k_{t}+1}$; and membrane position $\mathbf{X}_{k_{t}+1}$ at time $t_{k_{t}+1}$ are computed using the following steps: 
a. Solve the velocity and pressure fields $\mathbf{u}_{k_{t}+1}$ and $p_{k_{t}+1}$ at time $t_{k_{t}+1}$ keeping the capsule configuration $\mathbf{X}_{k_{t}}$ and the membrane stresses fixed, at the value computed at time $t_{k_{t}}$. The non-linear system $\mathbf{R}=\left[R_{m i}, R_{m c i}\right]^{T}=\mathbf{0}$ is solved using the Newton-Raphson method, and for each iteration, the linear system of equations is solved using the MATLAB ${ }^{\circledR}$ direct solver [50].

b. Interpolate the velocity field $\mathbf{u}_{k_{t}+1}$ using the quadratic inverse mapping to evaluate the velocity at each membrane node $\mathbf{X}_{k_{t}}$.

c. Update the position of the membrane nodes by integrating eq. (3-22), in order to find $\mathbf{X}_{k_{t}+1}$.

d. Return to "Step a." until $k_{t}$ reaches the total number of time steps. 


\section{Results}

Numerical results obtained in this work are presented in this chapter. For clarity, results are divided into four sections as follows: simple shear flow, constricted channel flow, straight capillary flow, and constricted capillary flow. Validation of formulation and numerical implementation is carried out in simple shear flow and straight capillary flow sections, in order to test code implementation for the 2-D planar flow and the axisymmetric flow, respectively.

\section{1}

\section{Validation: Simple shear flow}

Formulation and implementation are validated by comparing predictions of capsule deformation in simple shear flow with results available in the literature.

The flow domain considered is a square of length $L=16 a=1 \mathrm{~mm}$, as shown in Fig. 5.1, where $a$ is the initial capsule radius. The boundary conditions considered were: wall velocity $V_{\text {wall }}$ is imposed on upper and lower surfaces as $\left(+V_{\text {wall }}, 0\right)$ and $\left(-V_{\text {wall }}, 0\right)$, respectively. Additionally, along the left and right boundaries, fully developed flow, i.e. $\left.\mathbf{n} \cdot \boldsymbol{\nabla u}\right|_{x=0, L}=0$ and pressure equal to zero are defined, where $\mathbf{n}$ is the outwardly directed unit normal vector. Initially, an unstressed $\left(T_{0}=0\right)$ circular capsule with radius $a$ is located at the center of the domain. Upper and lower plates move in opposite directions with constant velocity $V_{\text {wall }}$, defining the shear rate $k=2 V_{\text {wall }} / L$.

The dimensionless parameters that govern the capsule deformation are the dimensionless shear stress $\Gamma[25]$ and the Reynolds number Re, defined as

$$
\Gamma=\frac{\mu k a}{E h}
$$




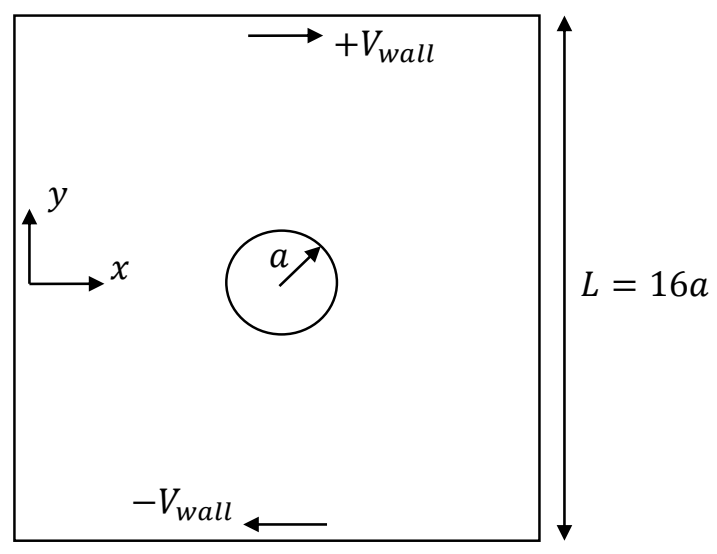

Figure 5.1: Simple shear flow domain.

$$
R e=\frac{\rho k a^{2}}{\mu}
$$

where $\Gamma$ and $R e$ are defined as the ratio of viscous to elastic forces and the rate of inertia to viscous forces, respectively. Furthermore, $\mu$ is the suspending phase viscosity, $E$ is Young's modulus, $h$ is the membrane thickness, and $\rho$ is the suspending phase density.

In these cases, the inner and outer phases have the same density $\rho=1000 \mathrm{~kg} / \mathrm{m}^{3}$ and viscosity $\mu=0.001$ Pa.s, and the membrane material has Poisson's ratio $\nu=0$ and a thickness $h=0.01 \mathrm{~mm}$.

The dimensionless shear stress $\Gamma$ and the Reynolds numbers $R e$ play an important role in determining the Taylor deformation parameter $D_{x y}$ and the capsule orientation with respect to the $x$-axis, $\theta_{x y}$. $D_{x y}$ is calculated as $(A-B) /(A+B)$, where $A$ and $B$ are the maximum and minimum axes of the deformed capsule, as illustrated in Fig. 5.2. Different values of $\Gamma$ and Re were explored by varying Young's modulus of the capsule membrane and the wall velocity $V_{\text {wall }}$.

In order to check grid resolution, three different meshes were tested at $\Gamma=0.0125$ and $R e=0.25$, as shown in Fig. 5.3. Notice that the mesh with $89 \times 89$ elements is good enough to obtain a mesh-independent solution, whilst the membrane grid varies dynamically around 176 Lagrangian points and the time step $\Delta t=1.5 \times 10^{-5} \mathrm{~s}$. On average, such number of Lagrangian points ensures that, at the initial time, 5 membrane nodes are defined per 


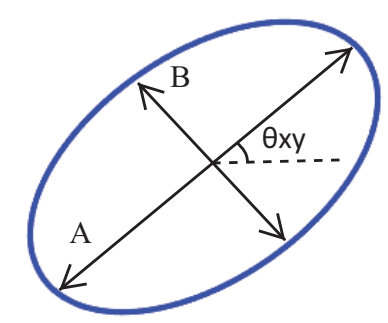

Figure 5.2: Definition of $A, B$, and $\theta_{x y}$.

quadrilateral element.

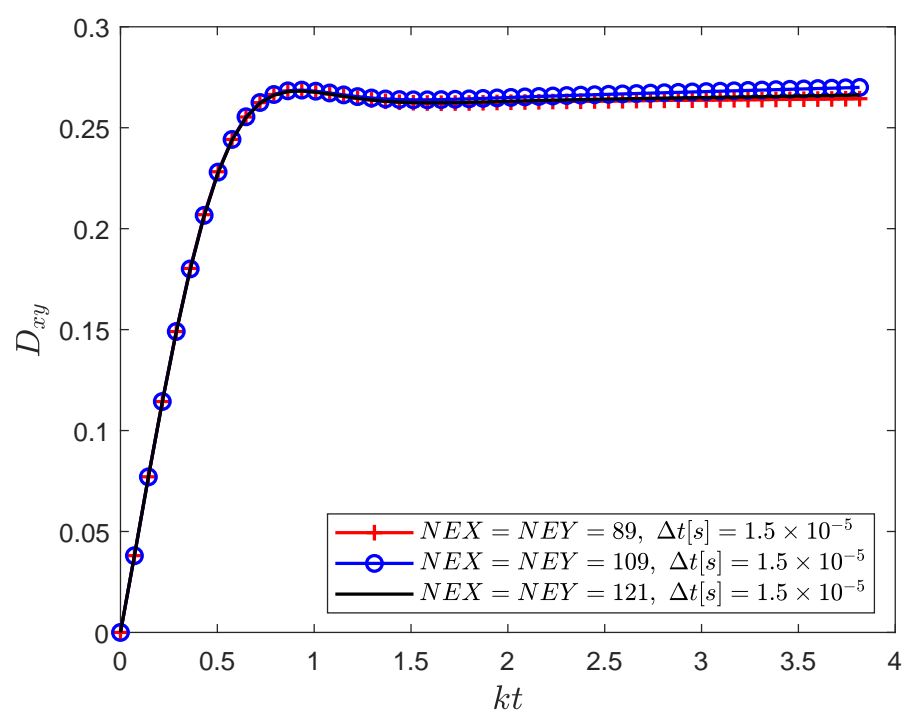

Figure 5.3: Capsule deformation for different grids at $\Gamma=0.0125$ and $R e=$ 0.25 .

Figure 5.4 presents the steady-state flow structure near the capsule and capsule configuration at $\Gamma=0.0125$ and $\Gamma=0.125$, at a fixed $R e=0.25$. As expected, the capsule deformation is larger and the inner pressure is lower at higher values of $\Gamma$ (lower Young's modulus). Moreover, both cases show that the pressure inside the capsule is higher than in the surrounding liquid.

Figure 5.5 presents the time evolution of the capsule deformation $D_{x y}$ for different values of dimensionless shear stress $\Gamma$. Present work results agree well with the results reported by Sui et al. [25], not only the steady-state regime but also the transient response. This is expected because Sui et al. [25] considered, similarly to this work, inertial effects. Both studies used the same Reynolds number, i.e. $R e=0.25$. The authors utilized a hybrid method combining immersed boundary and the lattice Boltzmann methods. The dif- 
(a)
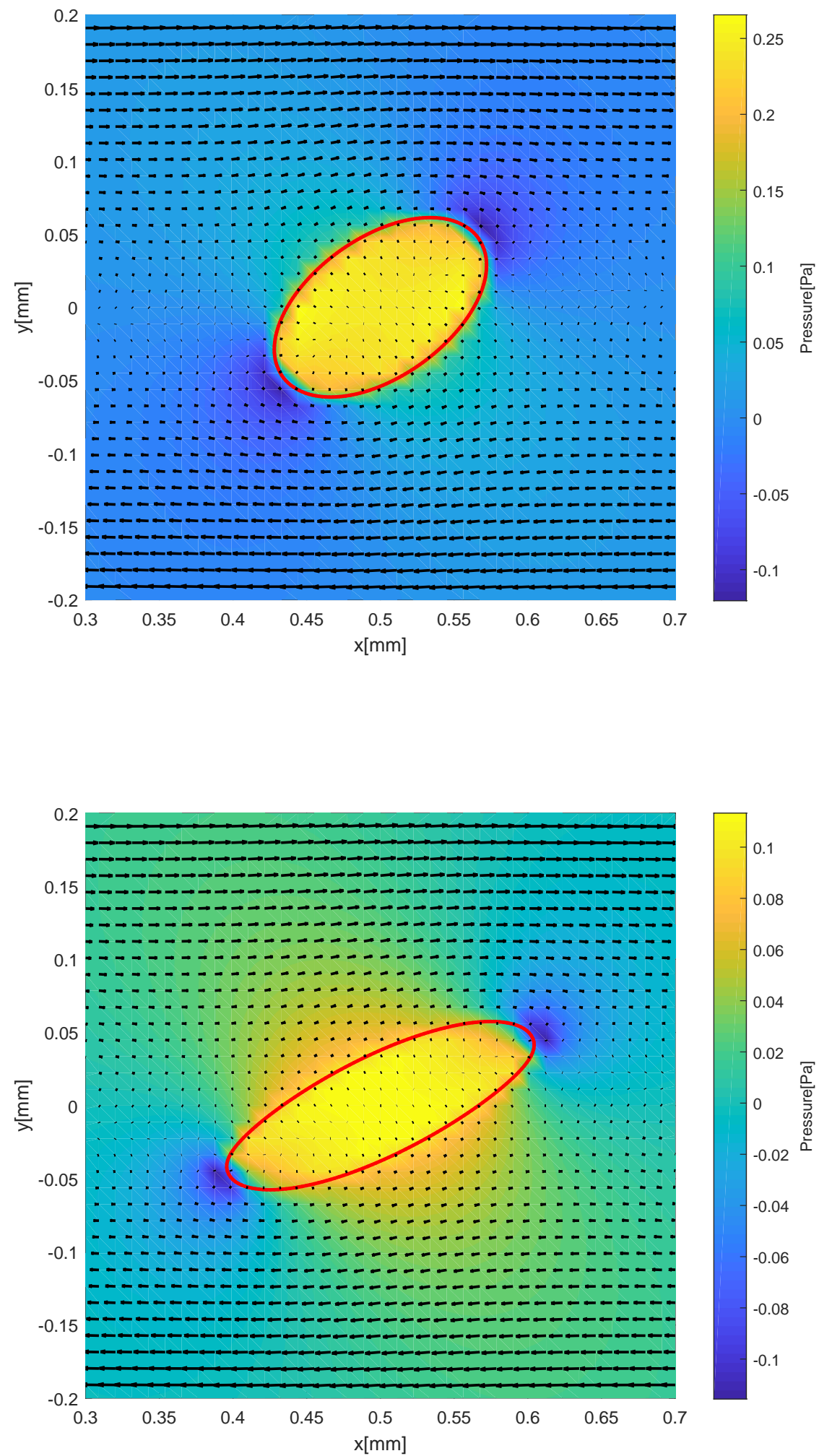

Figure 5.4: Steady-state flow structure and pressure field near the capsule at $R e=0.25$ and: (a) $\Gamma=0.0125$ and (b) $\Gamma=0.125$. 
ference between current results and Breyiannis \& Pozrikidis [22] results may be related to inertial effects that were neglected in their analysis. They used the boundary integral method to solve the Stokes flow problem.

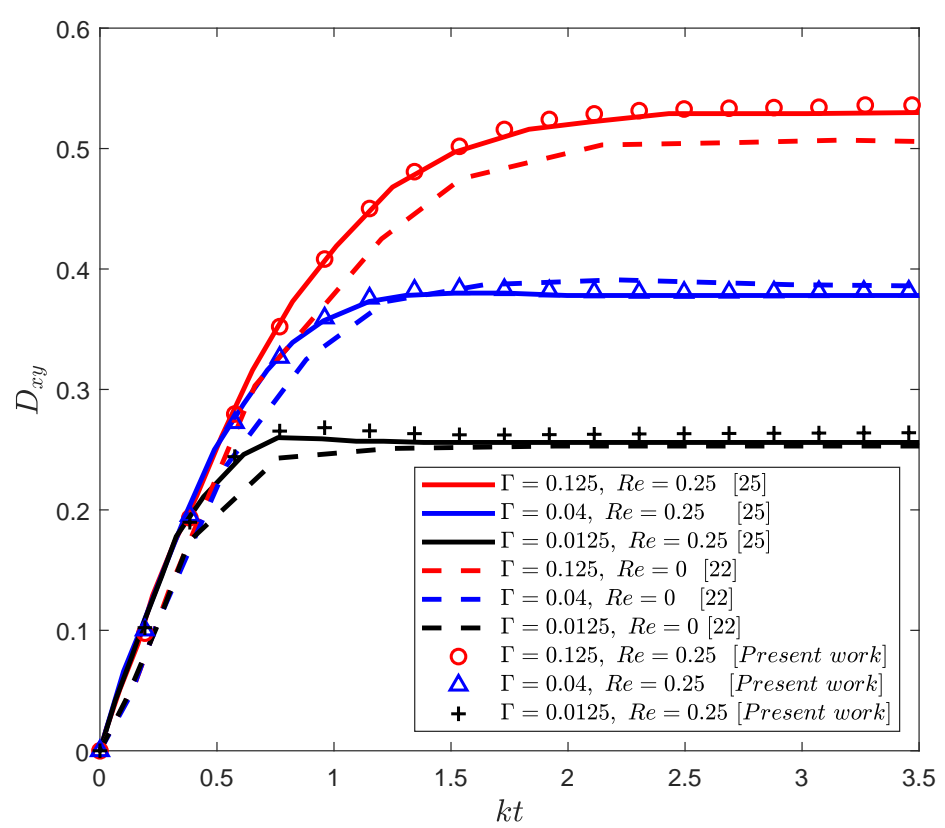

Figure 5.5: Temporal evolution of capsule deformation for different values of dimensionless shear stress $\Gamma$ and Reynolds number $R e$.

Figure 5.6 shows the time evolution of the capsule orientation $\theta_{x y}$ for different values of dimensionless shear stress $\Gamma$. Current results agree reasonably well with those reported by Sui et al. [25]. In fact, in the worst case scenario, i.e. $\Gamma=0.125$, the difference of capsule orientation between current results and the literature is less than $2^{\circ}$. 


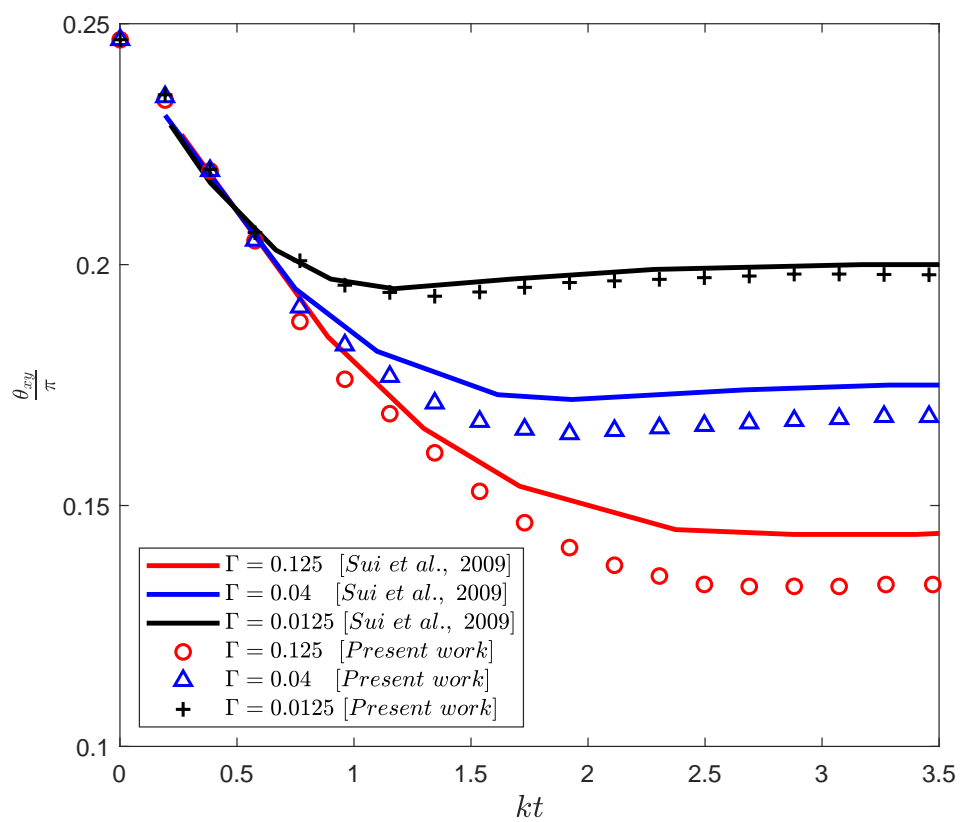

Figure 5.6: Temporal evolution of capsule orientation for different values of dimensionless shear stress $\Gamma$ at $R e=0.25$.

\section{2}

\section{Constricted channel flow}

After validating the formulation and numerical implementation for the 2-D problem, a planar channel flow with a constriction is studied. The main goal of the analysis is to determine the flow mobility (ratio of flow rate to pressure difference) reduction associated with the passage of a soft capsule through a constriction, which measures the ability of a capsule to partially block the flow through a pore throat and divert water towards oil-saturated pores.

Considering an imposed flow rate, the evolution of the pressure difference between inlet and outlet $\Delta P(t)=p_{\text {in }}(t)-p_{\text {out }}$ is computed for different capsule properties and flow conditions. The important dimensionless parameters are the following:

- Dimensionless capsule radius:

$$
\bar{a}=\frac{a}{H_{0}}
$$

- Preinflation parameter:

$$
\alpha=\frac{a}{a_{0}}-1
$$


- Constriction ratio:

$$
\beta=\frac{H_{0}}{H_{s}}
$$

- Surface capillary number:

$$
C a_{s}=\frac{\mu \bar{V}}{G_{s}}
$$

- Reynolds number:

$$
R e=\frac{\rho \bar{V} 2 H_{s}}{\mu},
$$

where $G_{s}$ is the surface shear modulus $[13,52]$, defined for materials obeying Hooke's law as $G_{s}=\frac{E h}{2(1+\nu)}$.

Throughout this section, the channel geometry is fixed in most cases. The entrance and constriction half channel heights are $H_{s}=0.5 \mathrm{~mm}$ and $H_{0}=0.215 \mathrm{~mm}$, i.e. the constriction ratio is $\beta=0.43$. The channel length is $L=4.5 \mathrm{~mm}$.

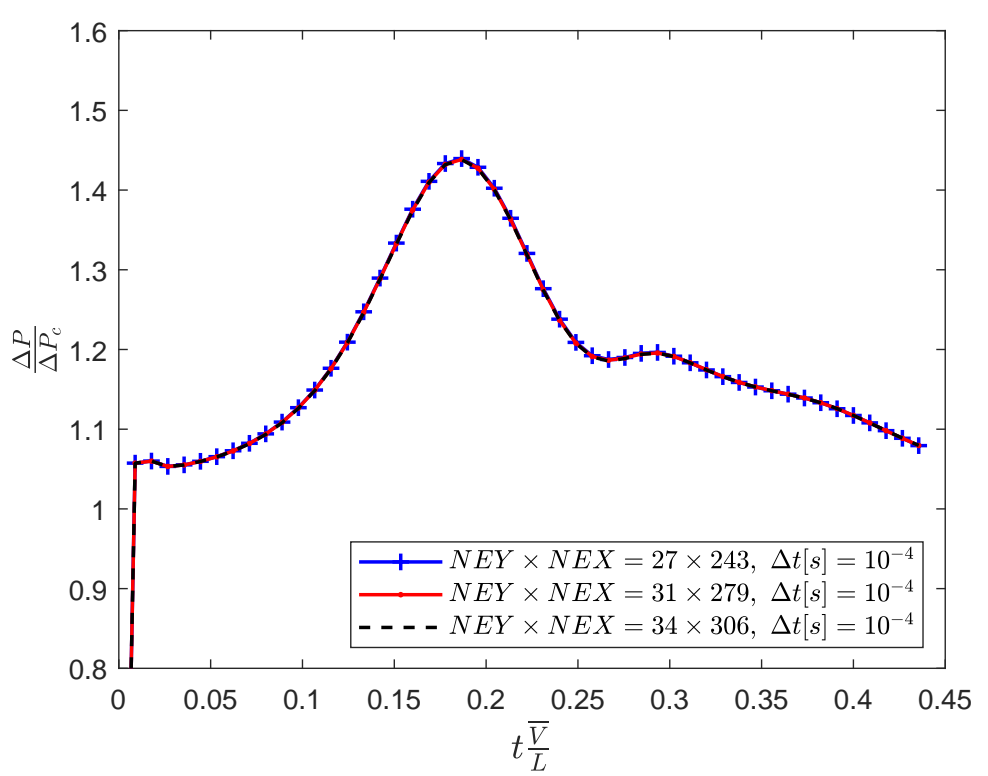

Figure 5.7: Temporal evolution of inlet pressure for different grids at $\bar{a}=1.63$, $\alpha=0.1, C a_{s}=0.05$, and $R e=1.0$.

The inner and outer liquid phases are the same, with density $\rho=1000 \mathrm{~kg} / \mathrm{m}^{3}$ and viscosity $\mu=0.001$ Pa.s. Therefore, the viscosity ratio $\lambda$, defined as the proportion of inner to outer phase viscosities, is fixed at 1 throughout this study. The membrane material has Poisson's ratio $\nu=0.499$ 
and a thickness $h=0.01 \mathrm{~mm}$.

A mesh of $27 \times 243$ quadrilateral elements and 238 Lagrangian points to define the 1-D solid domain (membrane) provide mesh-indepedent results, as shown in Fig. 5.7. It should be pointed out that instead of the pressure difference, another option could include the assessment of the mass of the fluid contained inside the membrane. However, some tests revealed that the mass of the fluid contained is much more sensitive to changes in the grid size than the pressure difference itself, therefore, an unsuitable variable to monitor for this study.

At initial time, the center of the capsule is placed at $x=0.45 \mathrm{~mm}$ and the liquid phase is at rest, as shown in Fig. 5.8. Due to the preinflation parameter $\alpha=0.1$, there is a pressure difference between inner and outer phases, equal to $T_{0} h / a$, as reported by Lefebvre and Barthès-Biesel [44]. This prestressed state plays an important role in capsule dynamics affecting its deformation, and probably attenuating undesired buckling instability. Three different preinflation parameters are explored, $\alpha=0.025,0.05,0.1$.

For a given channel geometry and Reynolds number $R e$, different values of capsule radii $\bar{a}$, preinflation parameters $\alpha$, and surface capillary numbers $C a_{s}$ are explored to determine their effect on the flow behavior. The parameter values explored are summarized in Table 5.1.

Table 5.1: Values of parameters for the constricted channel flow.

\begin{tabular}{|l|l|}
\hline Parameters & Values \\
\hline $\bar{a}$ & {$[1.63,1.86,2.33]$} \\
\hline$\alpha$ & {$[0.025,0.05,0.1]$} \\
\hline$\beta$ & {$[0.30,0.43]$} \\
\hline$C a_{s}$ & {$[0.005,0.01,0.02$,} \\
& $0.05,0.06,0.1,0.3,1.0]$ \\
\hline$R e$ & 1.0 \\
\hline
\end{tabular}

Figures 5.9, 5.10, 5.11, and 5.12 illustrate the temporal evolution of the flow field and the capsule configuration (red line in the plot) as it flows through the 2-D constricted channel at $\bar{a}=1.63, \alpha=0.1, R e=1.0$ for $C a_{s}=0.005, C a_{s}=0.02, C a_{s}=0.06$, and $C a_{s}=0.1$, respectively. As flow starts, the capsule moves towards the throat and deforms. The inlet pressure starts to rise in order to get the membrane deformed through the constriction, reaching its maximum when the capsule front end is close to 


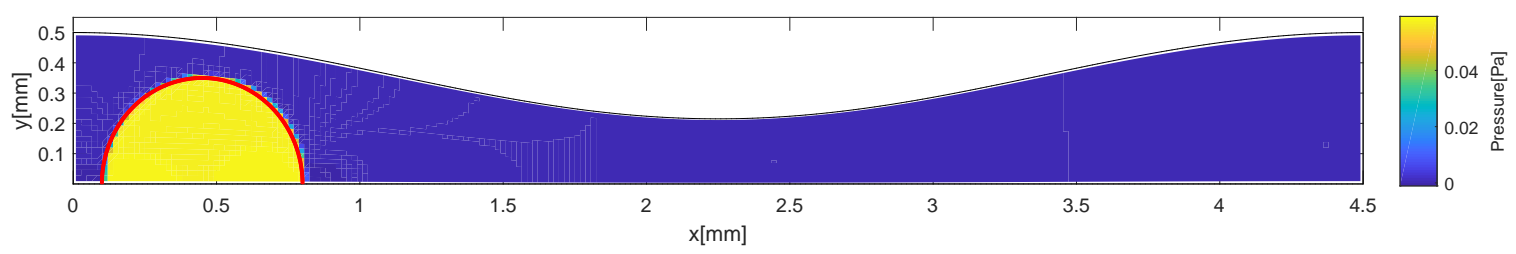

Figure 5.8: Initial pressure field and capsule configuration at $\bar{a}=1.63, \alpha=0.1$, $C a_{s}=0.02$, and $R e=1.0$.

(a)

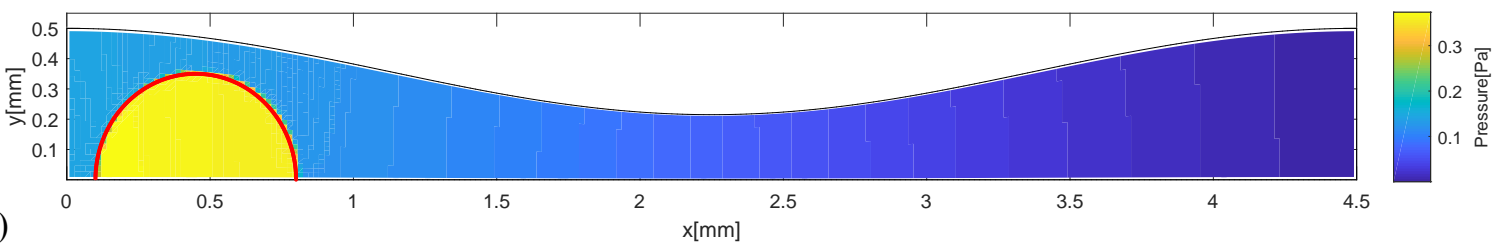

(b)
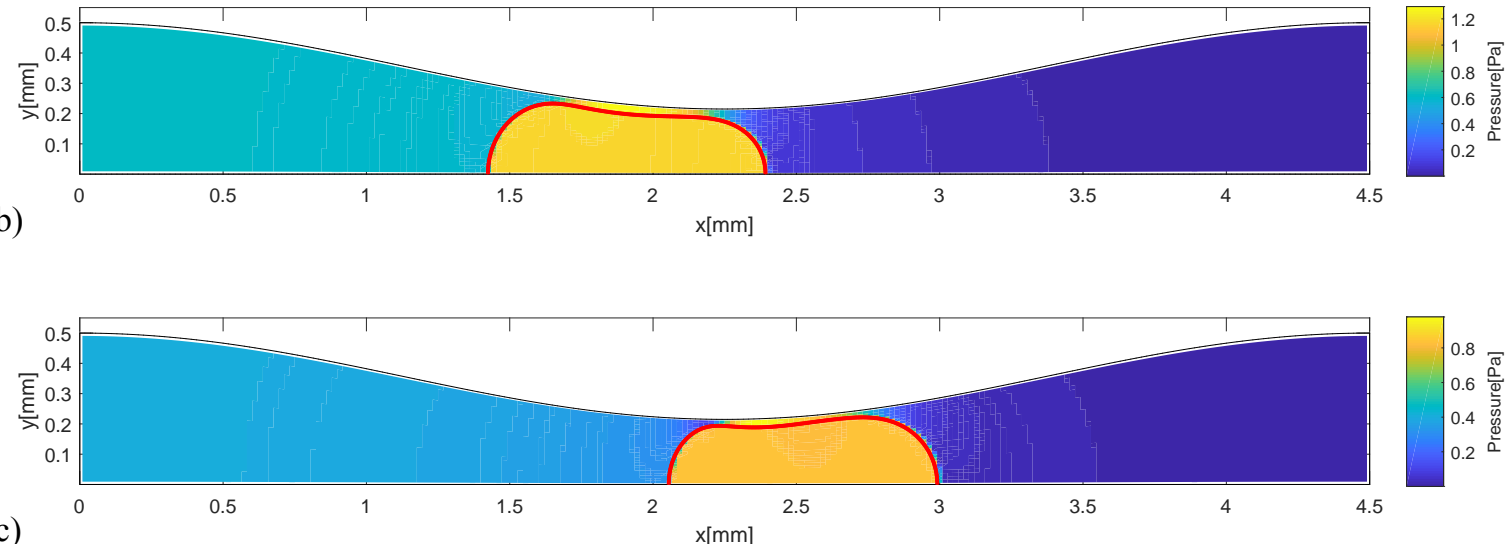

(d)

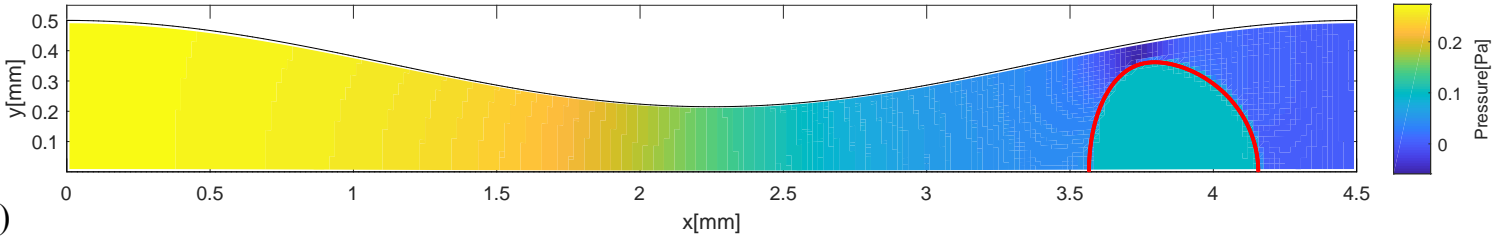

Figure 5.9: Temporal evolution of the membrane and pressure field: (a) $t=0.0001 \mathrm{~s}$; (b) $t=0.9233 \mathrm{~s}$; (c) $t=1.2 \mathrm{~s}$; and (d) $t=1.9999 \mathrm{~s}$ at $\bar{a}=1.63$, $\alpha=0.1, C a_{s}=0.005$, and $R e=1.0$.

narrower channel regions (second image of the respective cases). The inlet pressure falls as the capsule flows through the diverging section of the channel. Notice that, the region between the capsule and the channel wall, i.e. the thin liquid film, starts to pressurize as the capsule gets close to the constriction.

Membrane deformation depends on $C a_{s}$. At the lowest $C a_{s}=0.005$, the membrane is more rigid and the capsule shape is more circumferential at the diverging section. In fact, a convex shape is clearly observed at the 
(a)

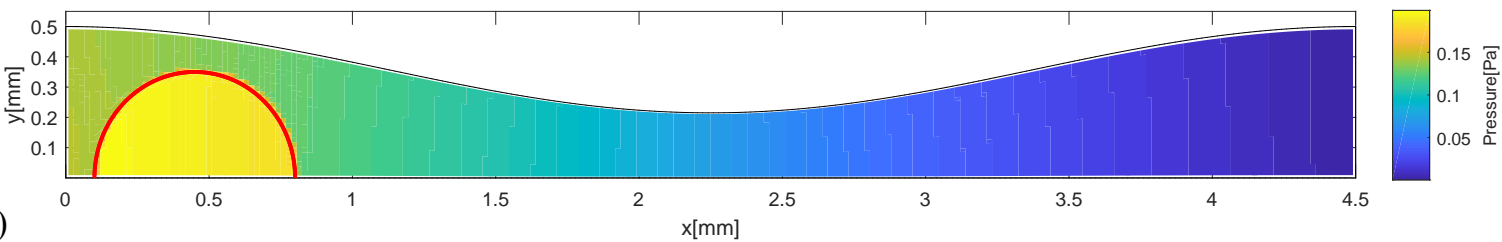

(b)
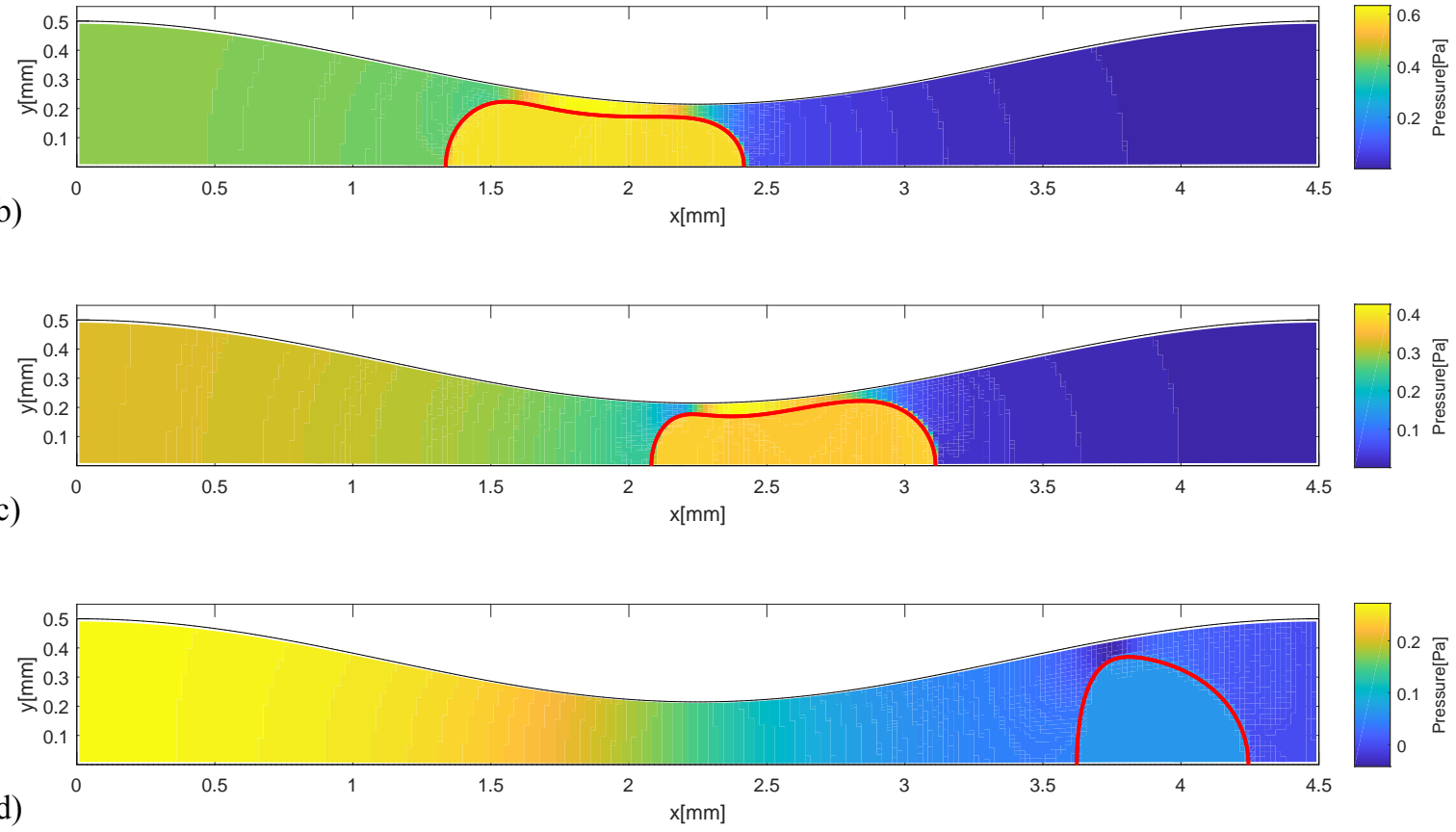

Figure 5.10: Temporal evolution of the membrane and pressure field: (a) $t=0.0001 \mathrm{~s}$; (b) $t=0.88 \mathrm{~s}$; (c) $t=1.2 \mathrm{~s}$; and (d) $t=1.9999 \mathrm{~s}$ at $\bar{a}=1.63$, $\alpha=0.1, C a_{s}=0.02$, and $R e=1.0$.

capsule rear end after the constriction, as illustrated in Fig. 5.9-d. The liquid film between the capsule and the channel wall is very thin leading to a high pressure value in the lubricating film.

As the surface capillary number $C a_{s}$ rises, the elastic force weakens, the lubricating film thickens, and the rear end of the capsule slightly starts to flatten after the constriction, as seen in Fig. 5.10-d.

Figure 5.11 illustrates the capsule evolution at the surface capillary number $C a_{s}=0.06$. It is observed that the lubricating film is even thicker than the previous case, and at the diverging region the capsule rear end is completely flat leading to a negligible pressure difference between inner and outer phases.

Figure 5.12 shows that at higher surface capillary number, e.g. $C a_{s}=0.1$, the capsule starts to get a croissant-like shape (concave shape) after passes 
(a)

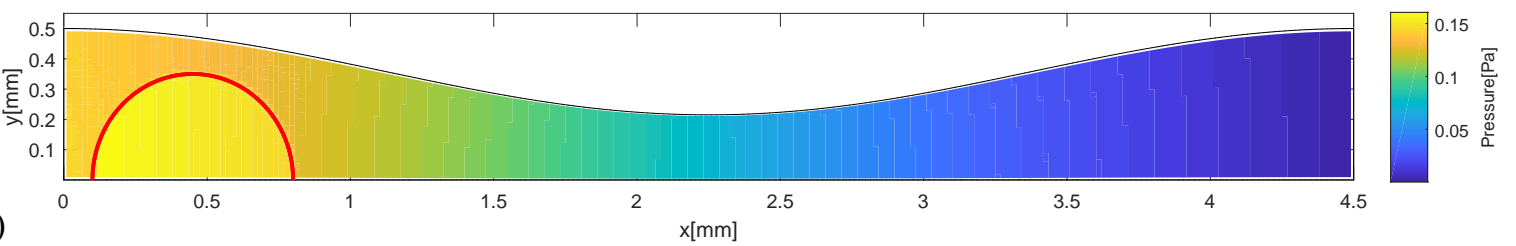

(b)

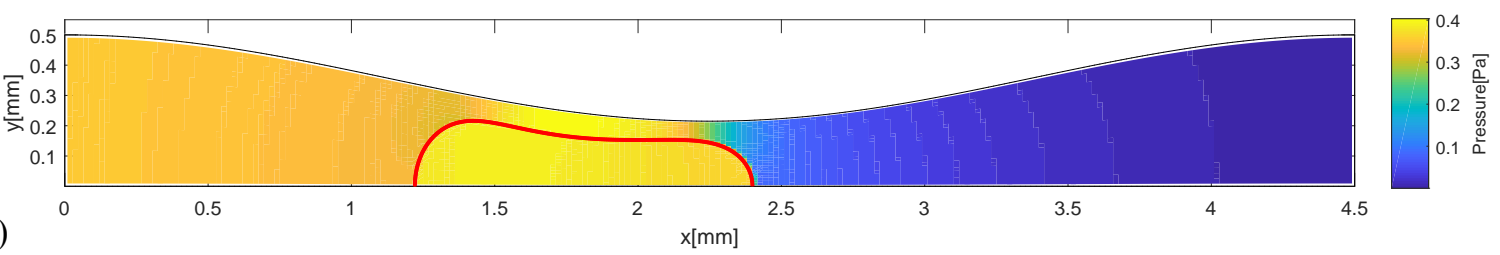

(c)
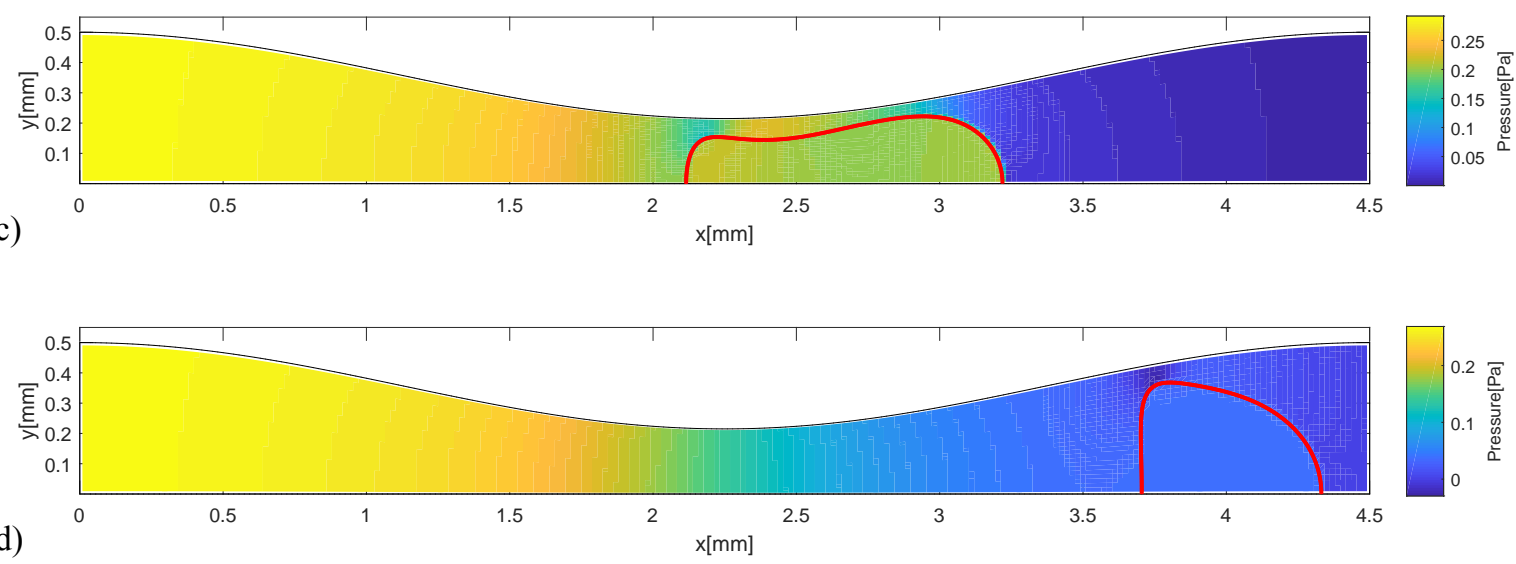

Figure 5.11: Temporal evolution of the membrane and pressure field: (a) $t=0.0001 \mathrm{~s}$; (b) $t=0.8235 \mathrm{~s}$; (c) $t=1.2 \mathrm{~s}$; and (d) $t=1.9999 \mathrm{~s}$ at $\bar{a}=1.63$, $\alpha=0.1, C a_{s}=0.06$, and $R e=1.0$.

through the constriction. Observe that at the capsule rear end, the curvature radius is so high that the pressure difference between inner and outer phases is negative but small.

There is a small loss of mass of the fluid contained inside the membrane, as illustrated in Figs 5.9-d, 5.10-d, 5.11-d, and 5.12-d. This is a well-known problem observed in the context of the immersed boundary method, as pointed out by Peskin \& Printz [53]. For the mentioned cases, the average mass loss is acceptable and below $-6.5 \%$, with the worst performance $-13 \%$ at the lowest $C a_{s}=0.005$. This might be attenuated by diminishing the time step and/or refining the mesh grid even further.

Stresses along the membrane arc-length at different times are presented in Fig. 5.13-a. Note that $l_{s}(t)$ is the half of the total membrane length. The corresponding membrane position and configuration at each time are illustrated in Fig. 5.13-b. At $t=0$, the membrane has a circumferential shape, 
(a)

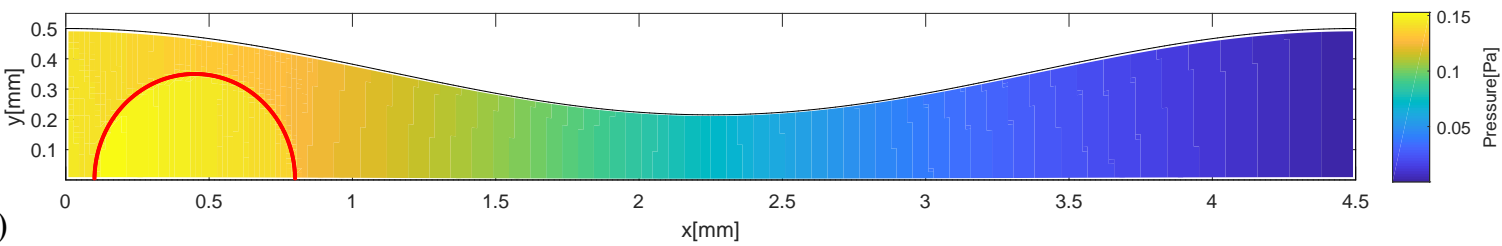

(b)

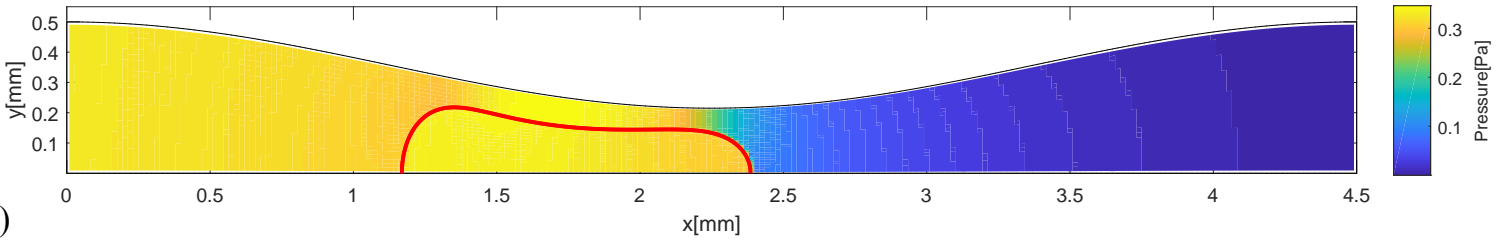

(c)
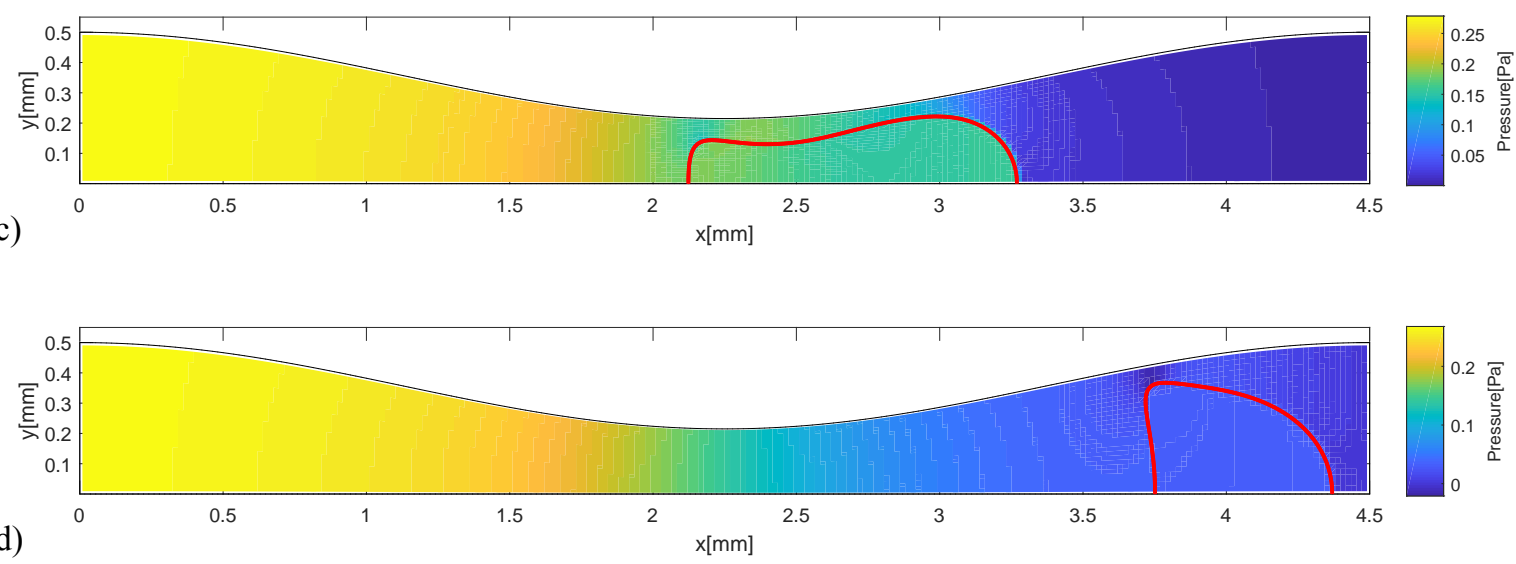

Figure 5.12: Temporal evolution of the membrane and pressure field: (a) $t=0.0001 \mathrm{~s}$; (b) $t=0.7956 \mathrm{~s}$; (c) $t=1.2 \mathrm{~s}$; and (d) $t=1.9999 \mathrm{~s}$ at $\bar{a}=1.63$, $\alpha=0.1, C a_{s}=0.1$, and $R e=1.0$.

and the stress is uniform and equal to the defined dimensionless prestress $\frac{T_{0}}{G_{s} / h}=0.399$. As time elapses, the capsule deforms and gets stretched in order to pass through the constriction. It is worth noting that near the membrane front region, higher stresses can be observed. This indicates that in cases at which the membrane ruptures, it does near the front end, as observed by Leopércio et al. [54]. After the constriction, the membrane starts to relax and the stress falls. At $t \frac{\bar{V}}{L}=0.33$, there is a discontinuous derivative in Fig. 5.13-a related to the inflexion point of the local curvature of the corresponding capsule profile in Fig. 5.13-b. As the capsule flows, some membrane segments are in compression when compared to the initial state. Furthermore, $T_{1}$ approaches $T_{0}$ without reaching it, because the capsule has already a steady-state profile (no circumferential).

In order to assist in the visualization of how the stress is distributed along the membrane, a colored-field for the stress at different times is presented in Fig. 5.14. As previously mentioned, at $t=0$, a uniform membrane 
(a)
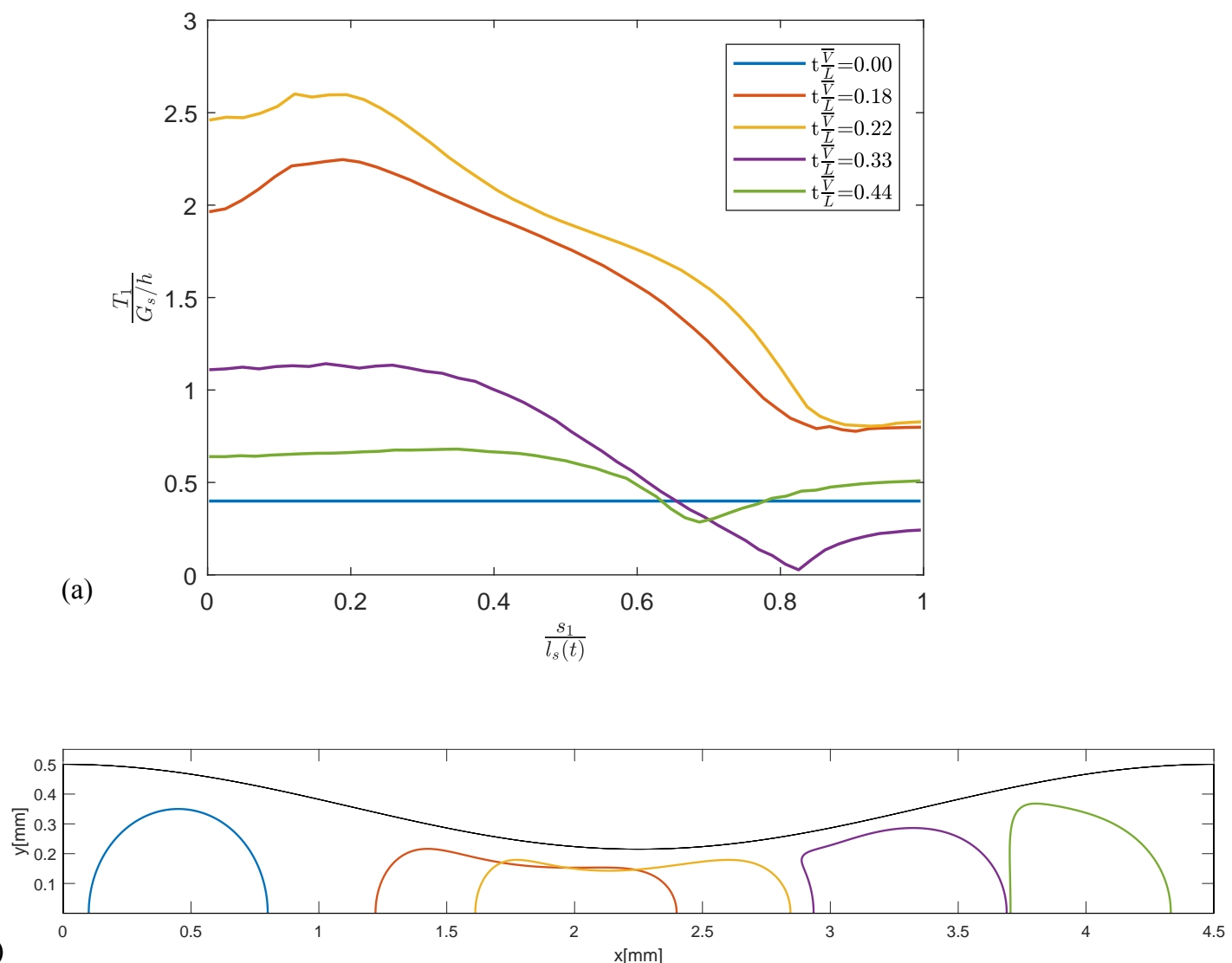

Figure 5.13: (a) Temporal evolution of stress along membrane arc-length at $\bar{a}=1.63, \alpha=0.1, C a_{s}=0.06$, and $R e=1.0$. (b) Position and membrane configuration at each time.

stress $T_{0}=0.665 P a$ is observed. As the capsule moves, front end membrane segments are under higher stress than those at the membrane rear end. Dimensionless time $t \frac{\bar{V}}{L}=0.22$ corresponds to the maximum membrane stress, at which the capsule center of gravity $X_{C G}$ almost reaches the throat, in this case $X_{C G}=2.23 \mathrm{~mm} \approx \frac{L}{2}$. As previously discussed, after the constriction, membrane stress falls with some membrane segments being compressed $\left(T_{1}<T_{0}\right)$.

Figure 5.15 illustrates the temporal evolution of the inlet pressure for different values of surface capillary number $C a_{s}$ rescaled by the steady-state pressure difference of continuous phase flow $\Delta P_{c}$. The dimensionless inlet pressure for a single-phase flow is the black continuous line, which is the solution for $C a_{s} \rightarrow \infty$. There is a small overshoot at the early time related to inertial effects associated with the flow startup. As expected, pressure rises as the capsule approaches the converging region of the channel, reaching its maximum value when the capsule front end is close to the narrowest region, and then falling to the value associated with the single-phase flow. Note that, 
(a)

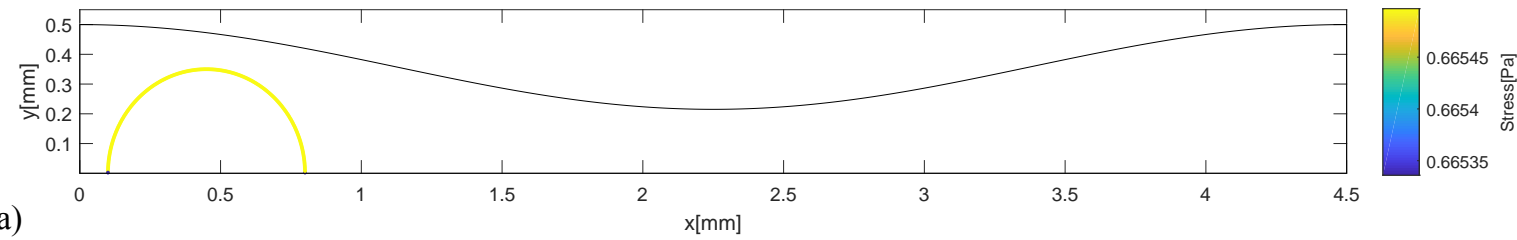

(b)

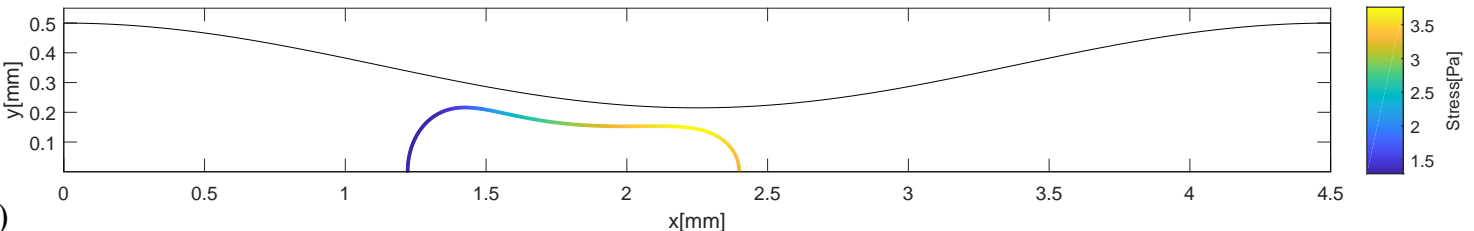

(c)
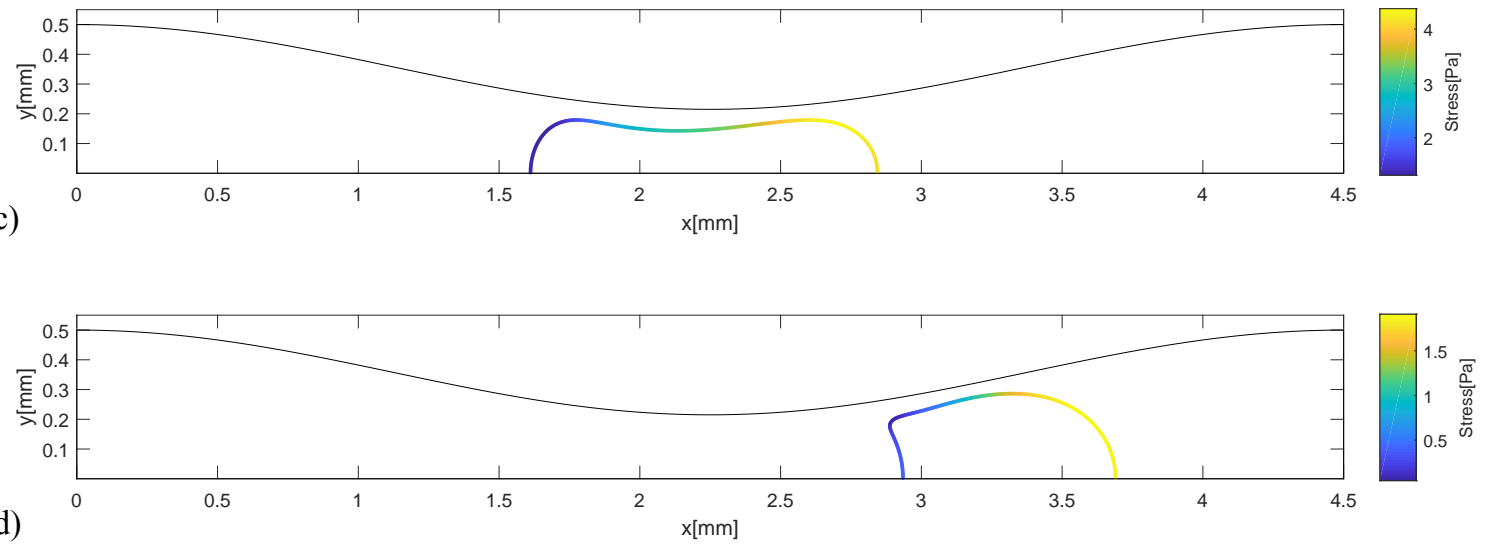

(e)

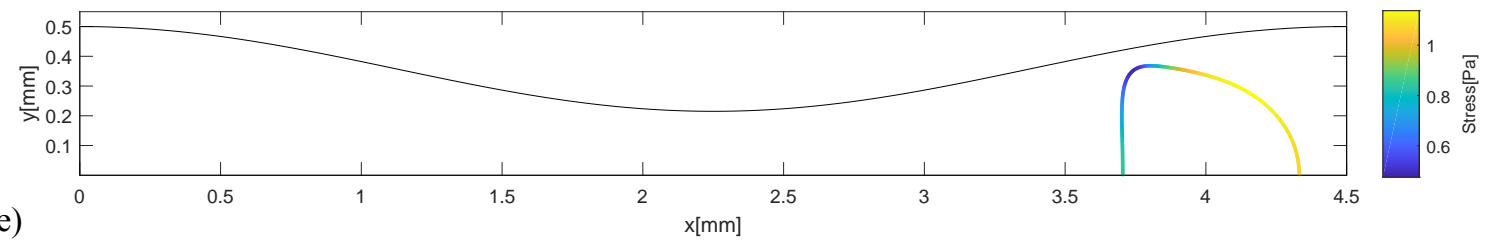

Figure 5.14: Stress colored-field at $\bar{a}=1.63, \alpha=0.1, C a_{s}=0.06$, and $R e=1.0$ : (a) $t \frac{\bar{V}}{L}=0.00$; (b) $t \frac{\bar{V}}{L}=0.18$; (c) $t \frac{\bar{V}}{L}=0.22$; (d) $t \frac{\bar{V}}{L}=0.33$; and (e) $t \frac{\bar{V}}{L}=0.44$.

this maximum delays as the surface capillary number $C a_{s}$ decreases. Although the three stages of pressure difference evolution, reported by do Nascimento et al. [8], have been observed, it is important to highlight that their experiments were conducted in a circular capillary and at values of surface capillary number $C a_{s}$ much lower than those reported here.

It is worth noting that the lower the surface capillary number $C a_{s}$, the higher the pressure peak, the greater the pressure needed to pull the capsule through the constriction; therefore, showing the key role played by the stiffness of the membrane material. 


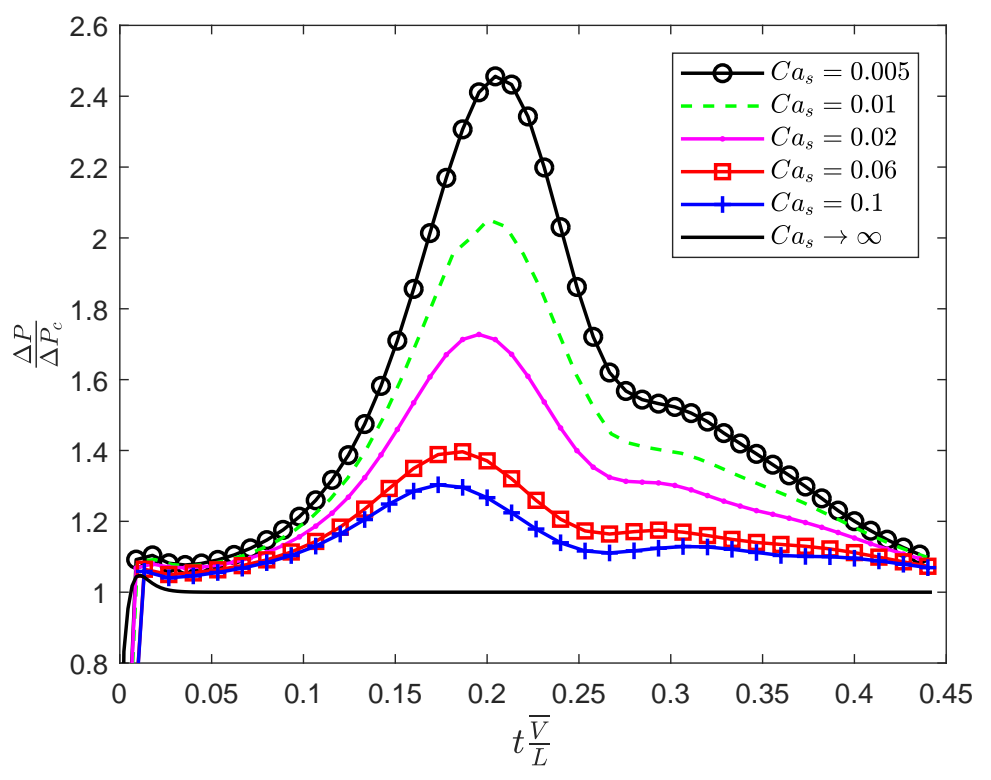

Figure 5.15: Temporal evolution of inlet pressure at $\bar{a}=1.63, \alpha=0.1$, and $R e=1.0$ for different values of surface capillary number $C a_{s}$.

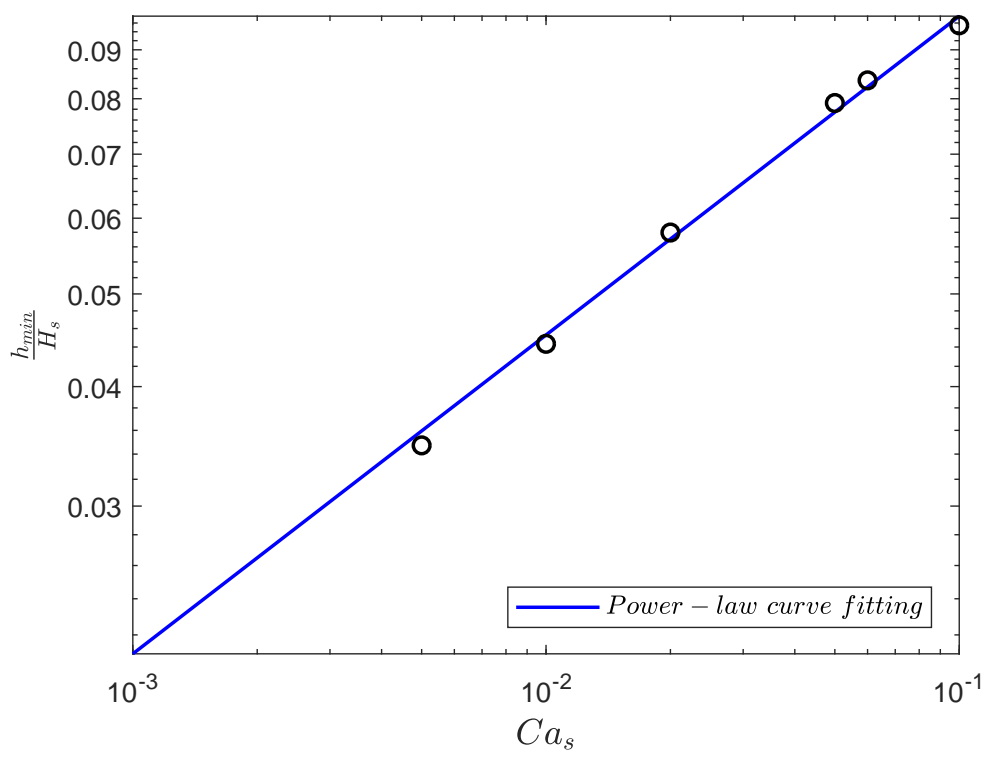

Figure 5.16: Minimum lubricating film thickness at $\bar{a}=1.63, \alpha=0.1$, and $R e=1.0$ as a function of surface capillary number $C a_{s}$.

The maximum pressure difference is related to the thickness of the thin liquid film between the membrane and the channel wall. As the capsule becomes more flexible, the minimum value of the liquid film thickness $h_{\text {min }}$ increases, as seen in Fig. 5.16. Considering $\bar{a}=1.63$ and $\alpha=0.1$, the minimum film thickness $h_{\text {min }}$ nondimensionalized by the inlet half channel height $H_{s}$ is 
well described by a power-law function:

$$
\frac{h_{\min }}{H_{s}} \approx C a_{s}^{0.33}
$$

Figure 5.17 shows the inlet pressure as a function of time at $\bar{a}=1.63$, $C a_{s}=0.06$, and $R e=1.0$ considering different values of preinflation parameter $\alpha$. The pressure peak slightly increases with the preinflation parameter $\alpha$. Higher prestressed level $T_{0}$ leads to stiffer capsules, requiring a higher inlet pressure to pull it through the constriction.

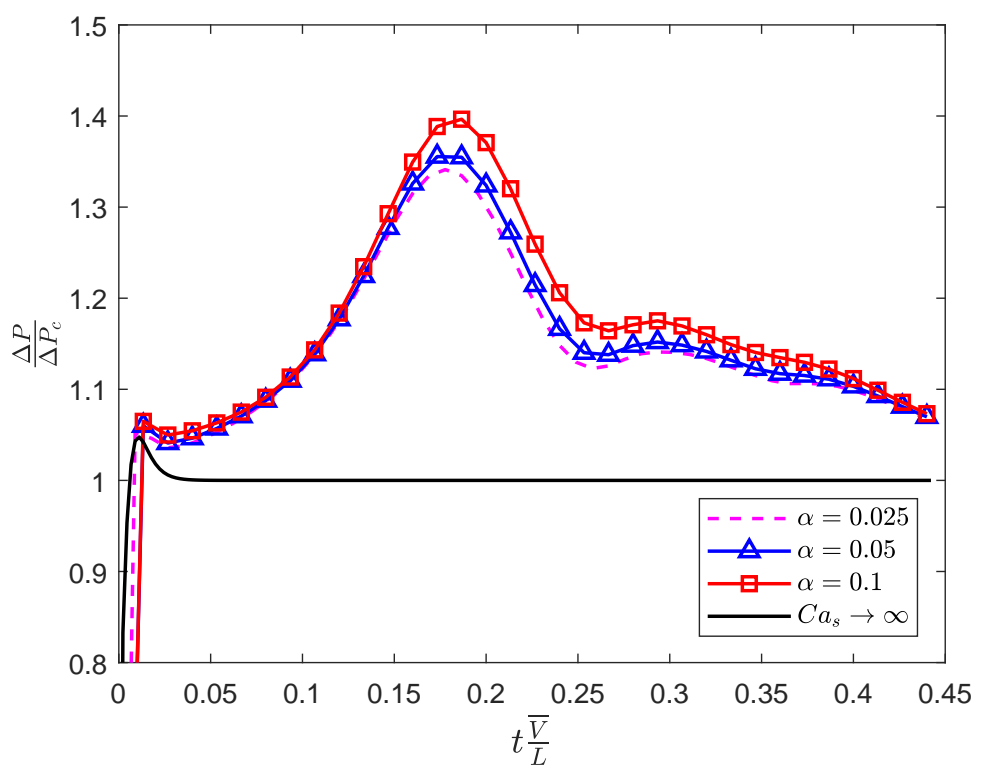

Figure 5.17: Temporal evolution of inlet pressure at $\bar{a}=1.63, C a_{s}=0.06$, and $R e=1.0$ for different values of preinflation parameter $\alpha$.

Figure 5.18 illustrates the combined effect of the dimensionless capsule radius $\bar{a}$ and the constriction ratio $\beta$ on the inlet pressure. For a given constriction ratio $\beta$, the larger capsule radius $\bar{a}$, the higher the normalized pressure peak. However, it is possible to obtain a similar maximum normalized pressure for two different capsule radii if the proper constriction ratio $\beta$ is used. For instance, the curve $(\bar{a}=1.86, \beta=0.43)$ produces a similar normalized pressure peak than the curve $(\bar{a}=2.33, \beta=0.30)$, showing the fundamental role of the constriction ratio.

Figure 5.19 presents the temporal evolution of the velocity of center of gravity of the capsule (hereupon capsule velocity) for different values of 


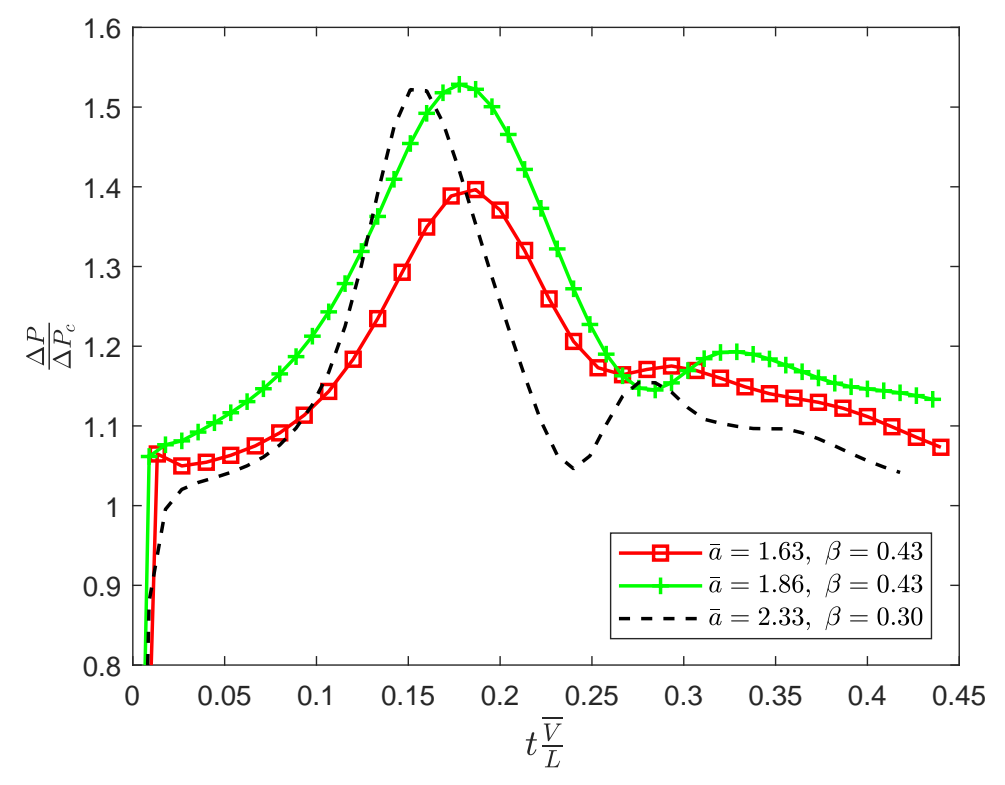

Figure 5.18: Temporal evolution of inlet pressure at $\alpha=0.1, C a_{s}=0.06$, and $R e=1.0$ considering different dimensionless capsule radii $\bar{a}$ and constriction ratios $\beta$.

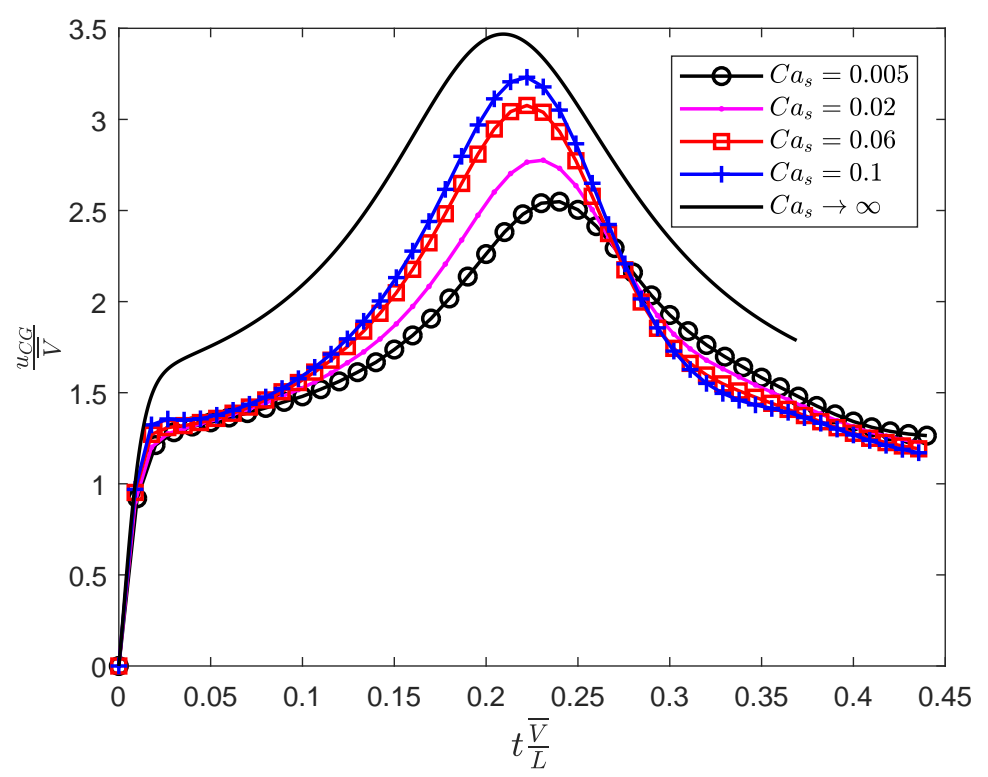

Figure 5.19: Temporal evolution of capsule velocity at $\bar{a}=1.63, \alpha=0.1$, and $R e=1.0$ for different values of surface capillary number $C a_{s}$.

surface capillary number $C a_{s}$. The case $C a_{s} \rightarrow \infty$ represents a situation in which the capsule does not affect the liquid phase, i.e. impose no force. Thus, the capsule velocity is the actual liquid velocity along the symmetry plane. Considering mass conservation, the dimensionless liquid velocity 
rises as the channel height falls, reaching its maximum at the throat, i.e. $u_{0} / \bar{V}=\left(u_{s} / \bar{V}\right) /\left(H_{0} / H_{s}\right)=1.5 / \beta=3.48$. As the capsule becomes more rigid and the surface capillary number falls, the capsule starts to affect the flow diminishing the capsule velocity, which signals partial blocking of the flow. At lower $C a_{s}$, the maximum capsule velocity diminishes, and the time at which the capsule reaches the throat is delayed.

Similarly to the work by Cobos et al. [55], a blocking factor $f$ is defined as the ratio of the pressure difference of the continuous phase flow to the maximum pressure difference of the suspended capsule flow at the same Reynolds number Re:

$$
f=\frac{\Delta P_{c}}{\Delta P_{\max }}
$$

The flow mobility reduction is characterized by $f$. A lower value of $f$ corresponds to a flow condition at which the elastic capsule strongly blocks the pore, whereas a higher value of $f$ means that the capsule has a weak effect on blocking. Figure 5.20 depicts the mobility factor $f$ as a function of surface capillary number $C a_{s}$, dimensionless capsule radius $\bar{a}$, and preinflation parameter $\alpha$. The partial pore blocking mechanism that divert water is effective if the local $C a_{s}$ is low enough. For a given $\bar{a}$ and preinflation parameter $\alpha$, the lower $C a_{s}$, the lower the factor $f$. Similarly, for a given value of $C a_{s}$ and preinflation parameter $\alpha$, the larger the capsule, the lower the factor $f$. 


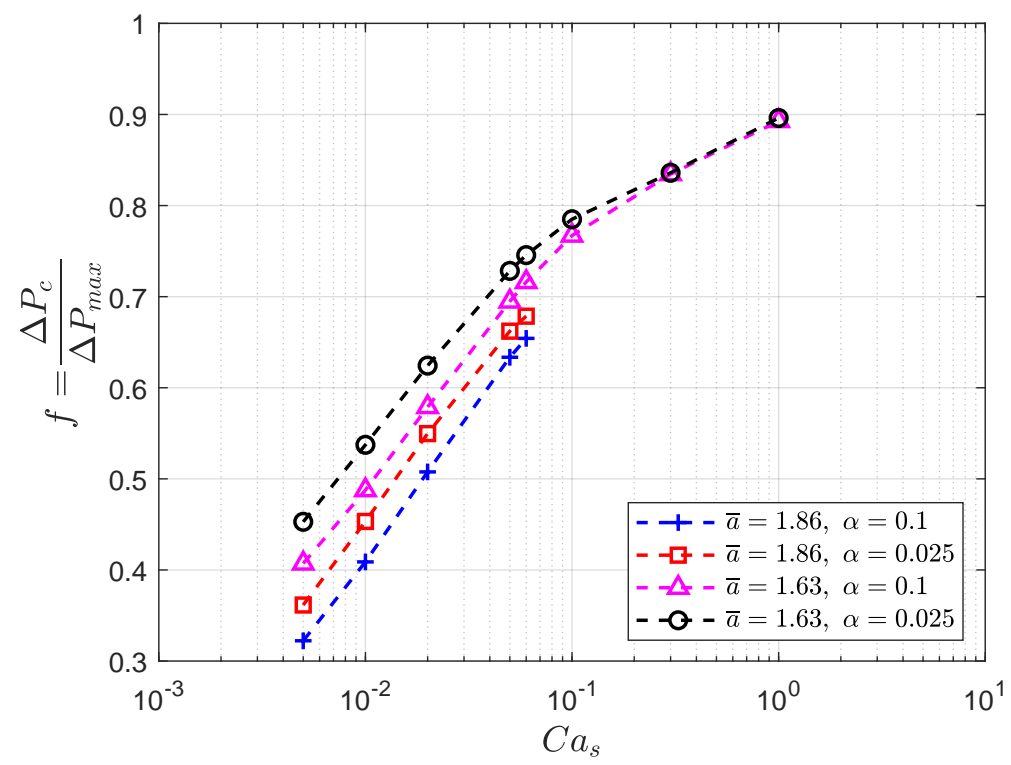

Figure 5.20: Mobility reduction factor $f$ as a function of $C a_{s}$ for different dimensionless capsule radii $\bar{a}$ and preinflation parameters $\alpha$ at $R e=1.0$.

\section{3}

\section{Validation: Straight capillary flow}

The objective of this section is to validate the axisymmetric formulation and implementation for capsule flow. For this reason, capsule deformation at steady-state through a straight capillary is compared with results available in the literature at different operating conditions, and different membrane materials are tested, such as Skalak's, Hooke's, and Neo-Hookean.

Boundary and initial conditions are similar to those defined in the constricted channel flow, including an imposed flow rate at the inlet. Furthermore, this problem almost shares the same dimensionless parameters with the previous section considering slight modifications as follows:

- Dimensionless capsule radius:

$$
\bar{a}=\frac{a}{R_{0}}
$$

- Constriction ratio:

$$
\beta=\frac{R_{0}}{R_{s}}
$$

- Reynolds number:

$$
R e=\frac{\rho \bar{V} 2 R_{s}}{\mu}
$$


where $R_{s}$ and $R_{0}$ are the inlet and constriction capillary radii, respectively. In this section, the constriction ratio is $\beta=1.0$, i.e. a straight capillary is considered. Dimensionless capsule radius $\bar{a}$, preinflation parameter $\alpha$, surface capillary number $C a_{s}$ and Reynolds number $R e$ determine the steady-state capsule deformation metrics, namely $L_{x}, L_{y}$, and $L_{f r}$, as illustrated in Fig. 5.21.

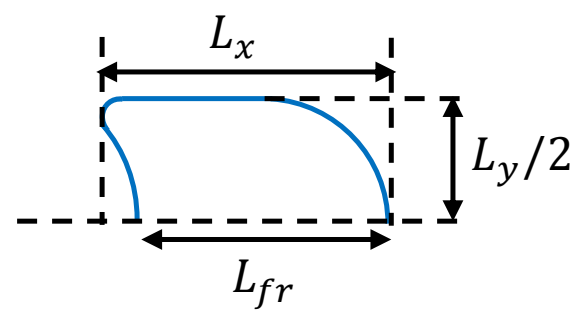

Figure 5.21: Definition of $L_{x}, L_{y}$, and $L_{f r}$.

Due to axisymmetry, a slice of the domain is enough to study the full flow. Thus, a section of dimensions $L=4.5 \mathrm{~mm}$ and $R_{s}=0.5 \mathrm{~mm}$ is defined. Figure 5.22 illustrates that mesh-independent solutions are obtained with $29 \times 261$ quadrilateral elements. In some cases, however, a finer mesh may be required. The membrane grid varies dynamically around 328 Lagrangian points and the time step $\Delta t=10^{-4} \mathrm{~s}$, in most cases. The inner and outer liquid phases have the same properties as in the previous section. The membrane thickness also remains as $h=0.01 \mathrm{~mm}$. The parameters used in the validation section are summarized in Table 5.2.

Table 5.2: Values of parameters for the straight capillary flow.

\begin{tabular}{|l|l|}
\hline Parameters & Values \\
\hline $\bar{a}$ & {$[0.8,0.9]$} \\
\hline$\alpha$ & {$[0.0,0.08,0.1]$} \\
\hline$\beta$ & 1.0 \\
\hline$C a_{s}$ & {$[0.02,0.03,0.05$} \\
& $0.1,0.24,0.40]$ \\
\hline$R e$ & {$[0.04,0.2,1.0,10.0]$} \\
\hline Membrane material & SK, HK, NHK \\
\hline
\end{tabular}

Capsule deformation metrics are obtained when the steady-state is reached, as shown in Fig. 5.23, for a Neo-Hookean membrane material. Both capsule profiles at different times are well superimposed.

Different authors solved capsule flow under Stokes flow conditions, as reported by Lefebvre \& Barthès-Biesel [44] and Hu et al. [31]. For Neo-Hookean 


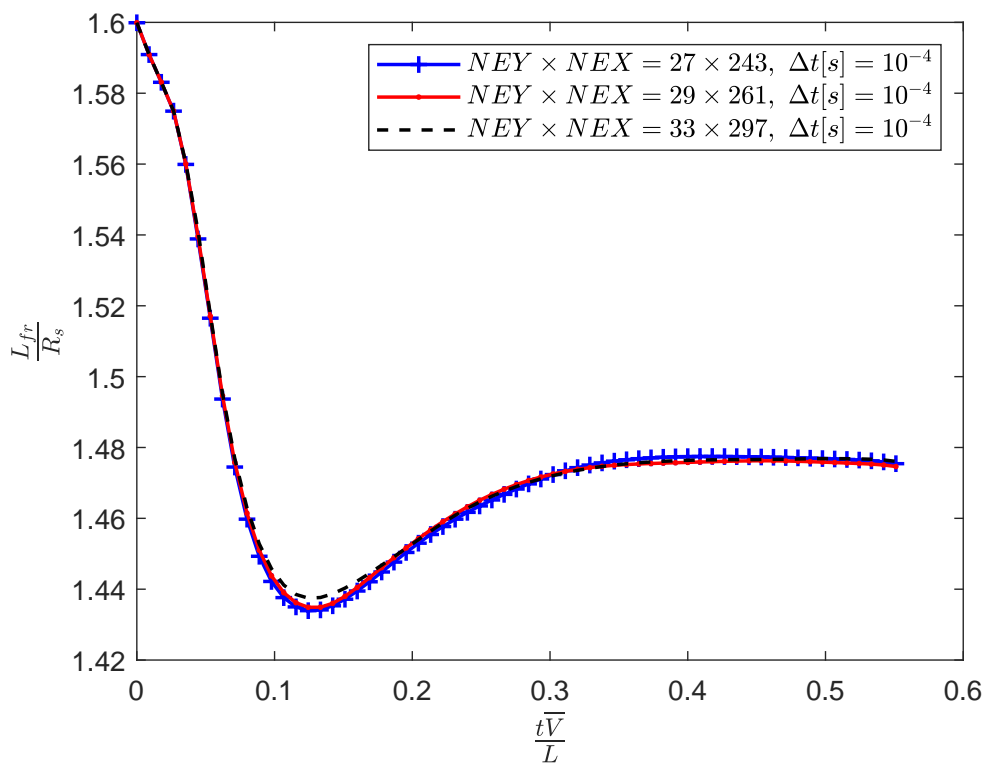

Figure 5.22: Temporal evolution of deformation $L_{f r}$ for different grids at $\bar{a}=0.8, \alpha=0.0, C a_{s}=0.24$, and $R e=1.0$.

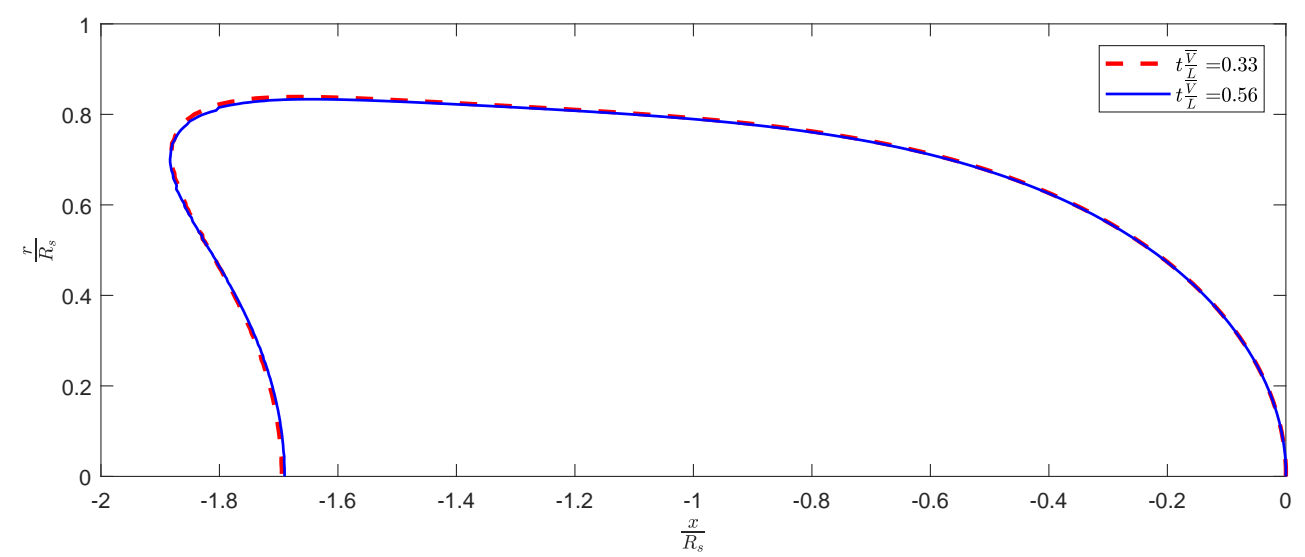

Figure 5.23: Steady-state reached by the capsule at $\bar{a}=0.9, \alpha=0.0$, $C a_{s}=0.05$, and $R e=1.0$.

membrane material, Fig. 5.24 illustrates the dimensionless deformation response $L_{x}$ for different values of $\operatorname{Re}$ at $\alpha=0.0$ and $C a_{s}=0.05$, in order to estimate the Reynolds number $R e$ below which the Stokes flow is recovered. Note that $R e=1.0$ can be used for such purpose, because the steady-state deformation $L_{x}$ for curves below that $R e$ value are nearly the same.

For Neo-Hookean membrane material, Fig. 5.25-a depicts dimensionless deformation $L_{x}$ and $L_{f r}$ for a range of $C a_{s}$ at $\bar{a}=0.9, \alpha=0.0$, and $R e=1.0$. Observe that comparison between present results and $\mathrm{Hu}$ et al. [31] predictions 


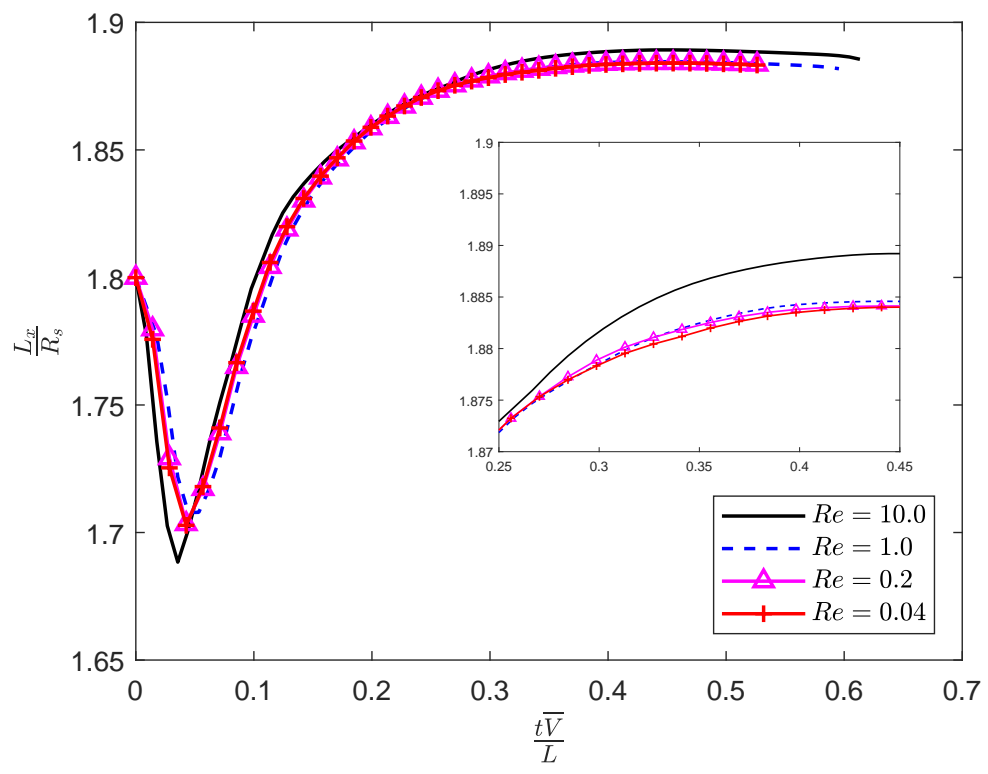

Figure 5.24: Temporal evolution of deformation $L_{x}$ for different values of $R e$ at $\bar{a}=0.9, \alpha=0.0$, and $C a_{s}=0.05$.

improves as $C a_{s}$ gets higher. The largest difference, found at $C a_{s}=0.02$, is less than $6 \%$. Furthermore, the steady-state capsule velocity is shown in Fig. 5.25-b along with results presented in the literature. Observe that, the results of the present study agree reasonable well with the literature for the circular section, having $2 \%$ difference as the worst case scenario.

Additionally, Fig. 5.26 shows two capsule profiles at $R e=1.0$, for $C a_{s}=0.05$ and $C a_{s}=0.10$. Note that, in both profiles, the capsule front matches well with the literature results. However, some differences can be observed at the rear region of the capsule, with special attention on the $C a_{s}=0.10$ case. For this case, the model here developed predicts further membrane stretching at fluid regions subjected to higher shear stress.

Figure 5.27 presents the time evolution of the meridional and azimuthal membrane stresses at $C a_{s}=0.05$ and $R e=1.0$. The plot also shows the predictions obtained by $\mathrm{Hu}$ et al. [31]. Observe that both results agree well in most of the membrane domain, although the presence of oscillations and a stress jump near the capsule tip $\left(s_{1}=0\right)$. The reason may be related to membrane grid discretization, boundary conditions, or even the time step used. Notice that in most part of the capsule profile, $T_{1}$ is larger than $T_{2}$, and some cross-sections of the capsule are in compression, i.e. $T_{2}$ is negative. 
(a)

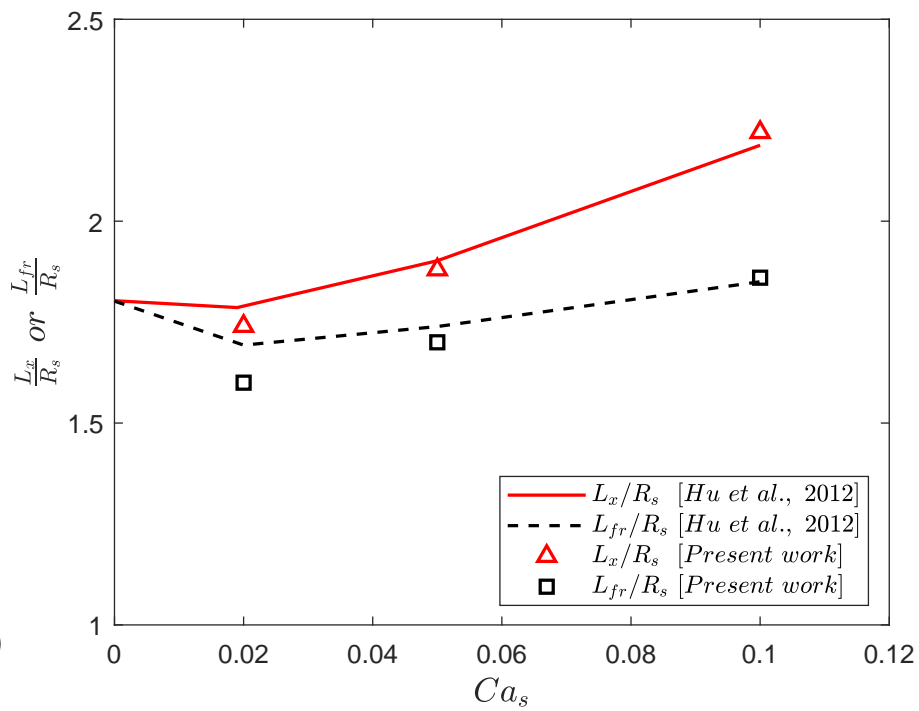

(b)

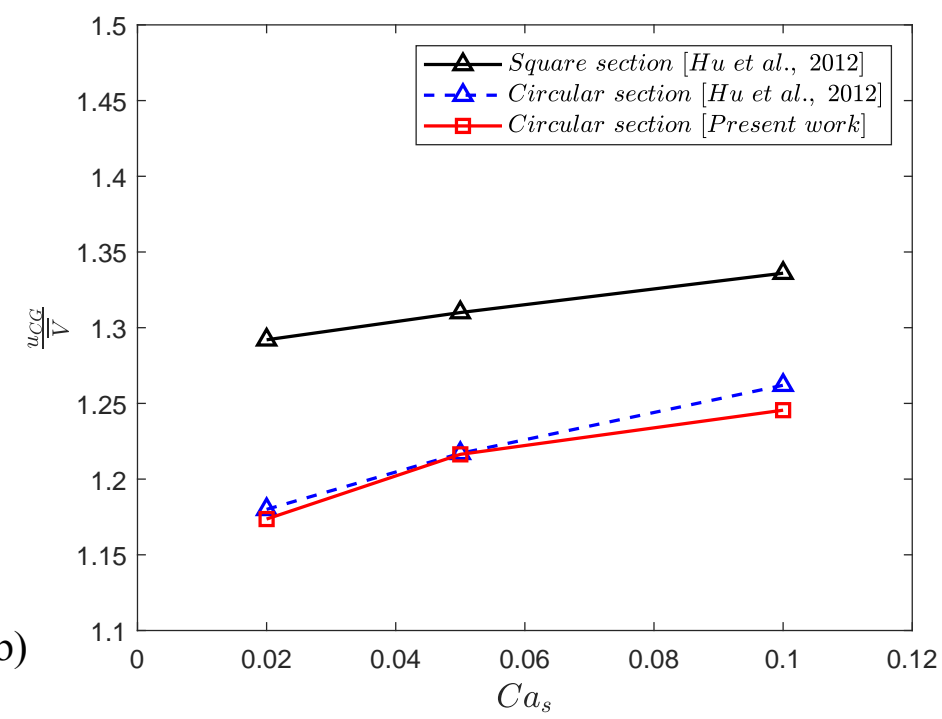

Figure 5.25: (a) Dimensionless $L_{x}$ and $L_{f r}$ as a function of $C a_{s}$. (b) Capsule velocity $u_{C G}$ as a function of $C a_{s}$ at $\bar{a}=0.9, \alpha=0.0$, and $R e=1.0$.

For Skalak's membrane material, comparison between present results and literature predictions is even better, probably because these tests are conducted at higher surface capillary number $C a_{s}$ than Neo-Hookean simulations. Figure 5.28 shows dimensionless deformation metrics for a wide range of surface capillary number $C a_{s}$ and preinflation parameter $\alpha$. Moreover, capsule profiles obtained in this work agree with profiles published by Lefebvre \& Barthès-Biesel [44], as clearly illustrated in Fig. 5.29.

Additionally, Carroll [13] numerically studied capsule flow considering 


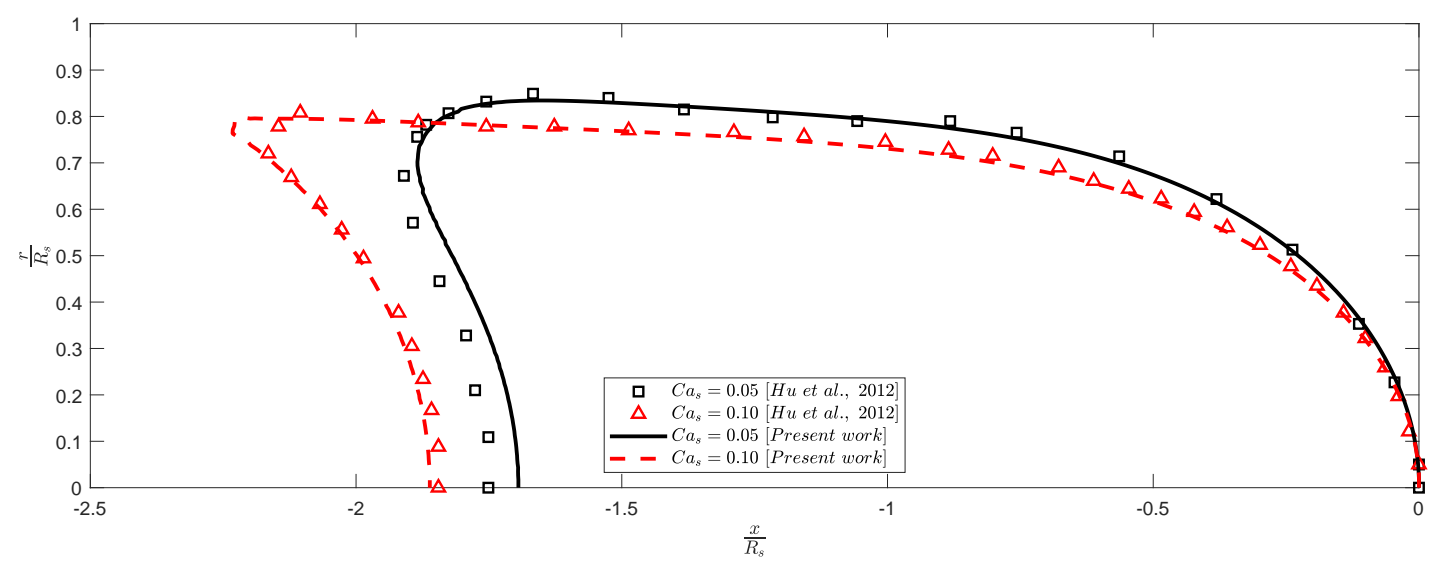

Figure 5.26: Comparison of capsule profiles at $\bar{a}=0.9, \alpha=0.0, R e=1.0$, for $C a_{s}=0.05$ and $C a_{s}=0.10$.

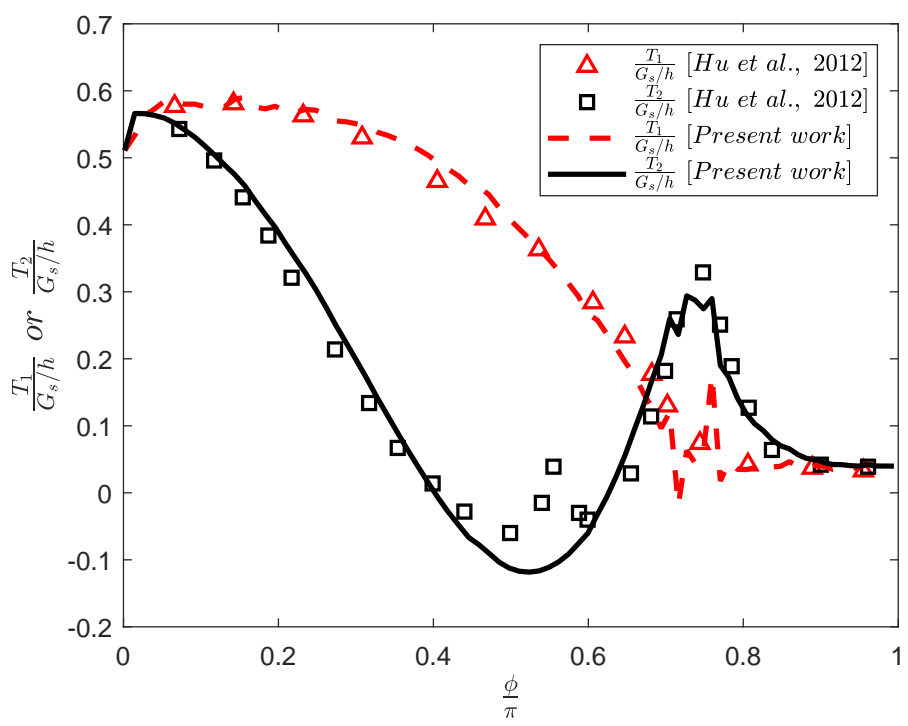

Figure 5.27: Dimensionless $T_{1}$ and $T_{2}$ as a function of meridional position $\phi$ at $\bar{a}=0.9, \alpha=0.0, C a_{s}=0.05$, and $R e=1.0$.

inertial effects, i.e. a finite Reynolds number $R e$, as in this work. Hence Fig. 5.30 compares results of this work with Carroll's results for Neo-Hookean membrane material. Observe that, for both preinflation parameters $\alpha$, the capsule front agrees well, whilst the capsule rear differs, in $x-r$ position, less than $3 \%$ on average.

Therefore, the model here developed and implemented agrees with available published data for Neo-Hookean and Skalak's membrane materials. Tests included different values of preinflation parameter $\alpha$, surface capillary number $C a_{s}$, and Reynolds number $R e$. 

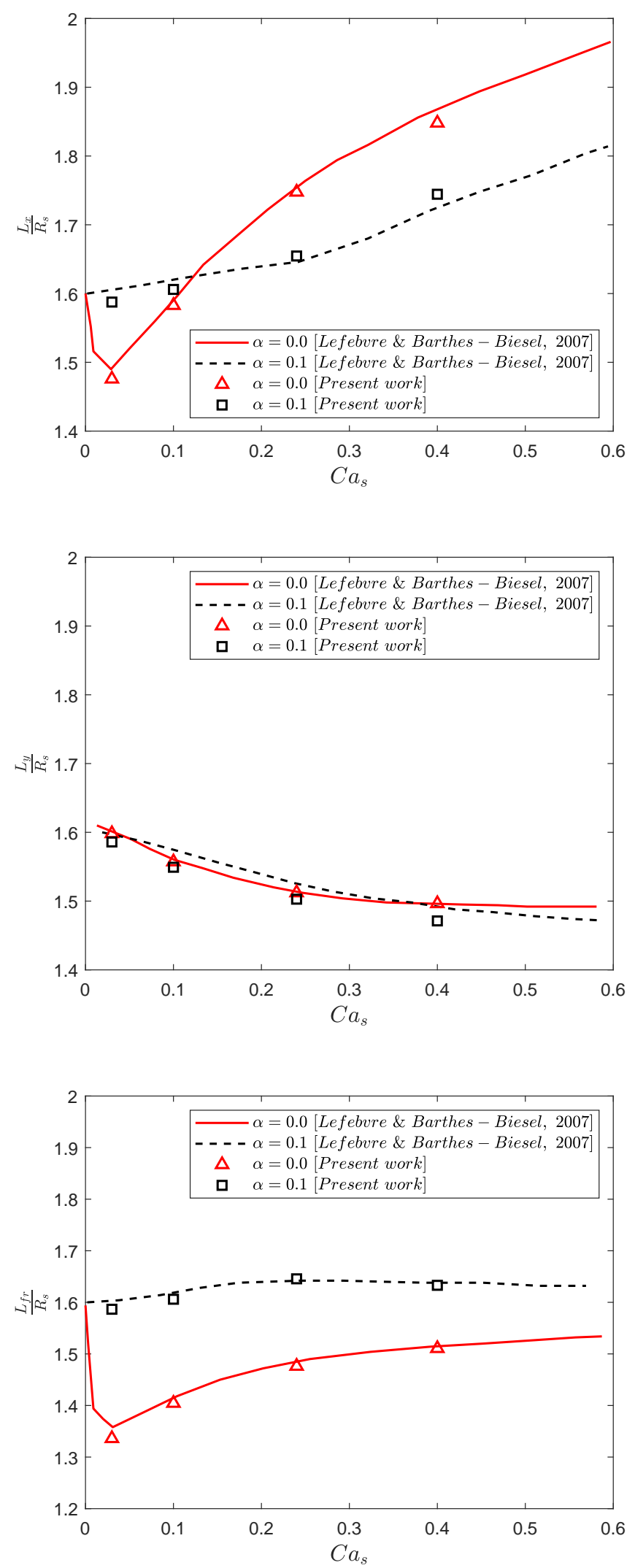

Figure 5.28: Dimensionless $L_{x}, L_{y}$, and $L_{f r}$ as a function of $C a_{s}$ at $\bar{a}=0.8$, $R e=1.0$, for $\alpha=0.0$ and $\alpha=0.1$. 


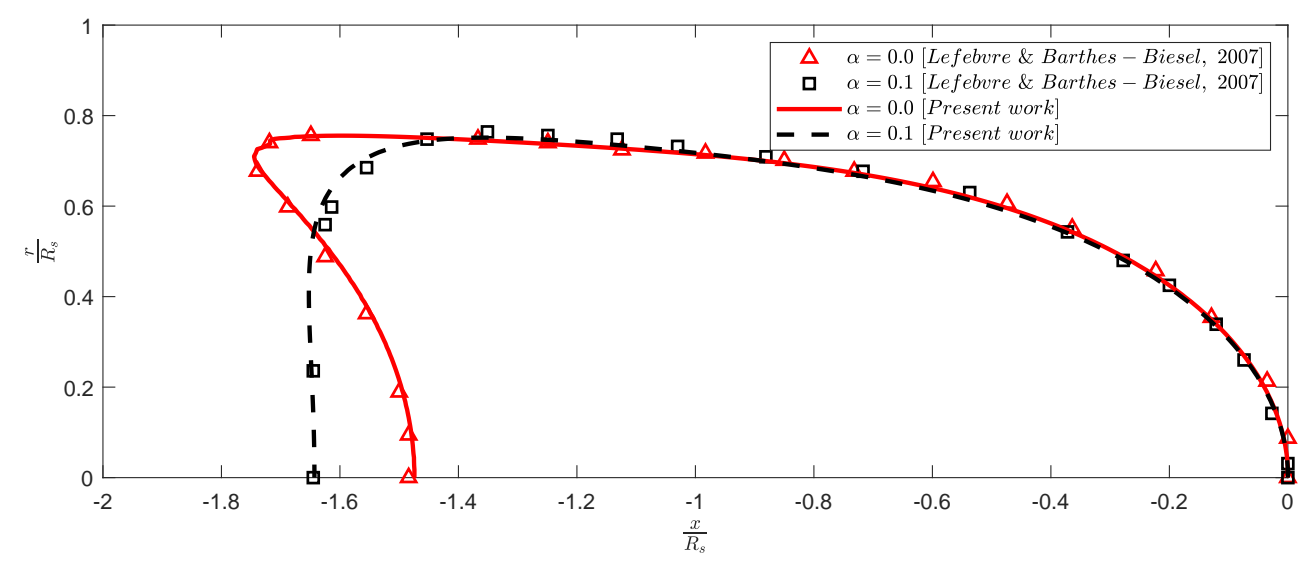

Figure 5.29: Comparison of capsule profiles at $\bar{a}=0.8, C a_{s}=0.24, R e=1.0$, for $\alpha=0.0$ and $\alpha=0.1$.

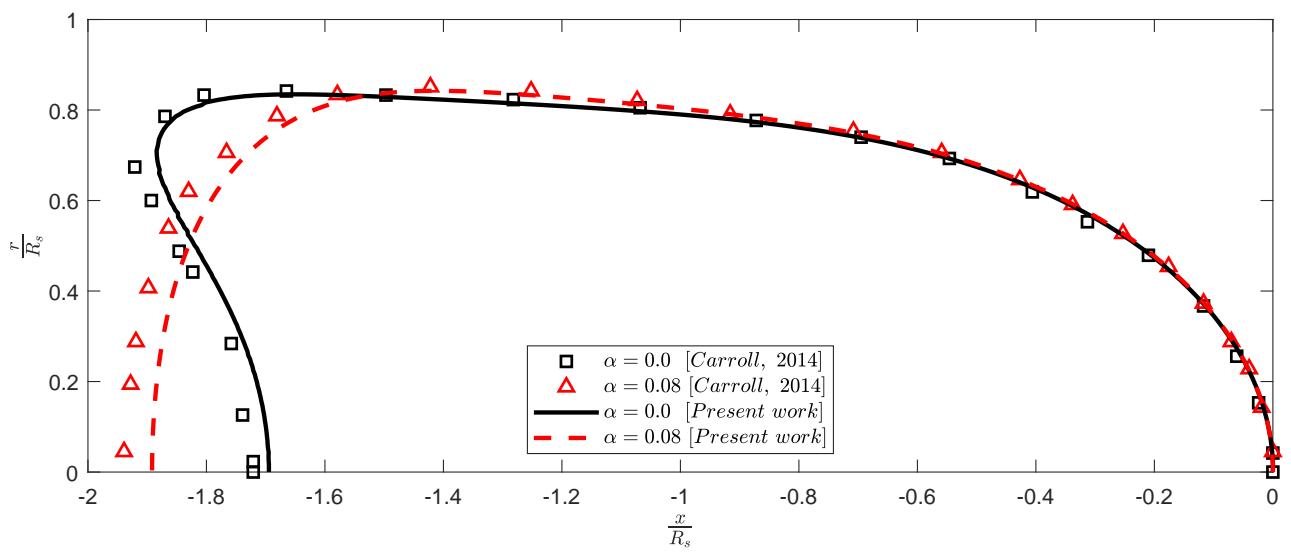

Figure 5.30: Comparison of capsule profiles at $\bar{a}=0.9, C a_{s}=0.05, R e=0.2$, for $\alpha=0.0$ and $\alpha=0.08$.

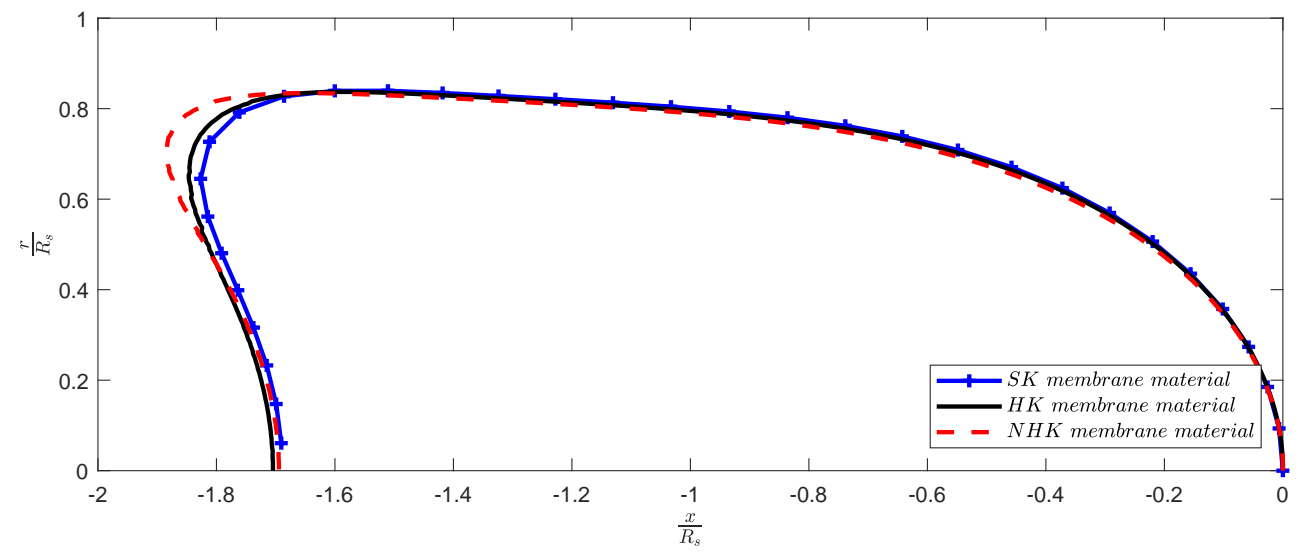

Figure 5.31: Comparison of capsule profiles at $\bar{a}=0.9, \alpha=0.0, C a_{s}=0.05$, and $R e=1.0$. Capsule membrane materials following Hooke's law, Skalak's, and Neo-Hookean equations. 
This section also includes some tests with membranes described by Hooke's law. Steady-state capsule profiles for Skalak's model, Hooke's law, and Neo-Hookean model materials are depicted in Fig. 5.31. They presented a similar front shape, and only differ at the rear region subjected to significant stress due to the flow. Observe that, the Skalak's membrane presents the lowest deformation, while Neo-Hookean membrane deforms the most at the same flow condition. Therefore, the Skalak's model shows a strain-hardening behavior, whilst the Neo-Hookean model has a strain-softening response.

Finally, regarding the pressure field, results with different membrane materials show very similar pressure fields, as seen in Fig. 5.32.

(a)
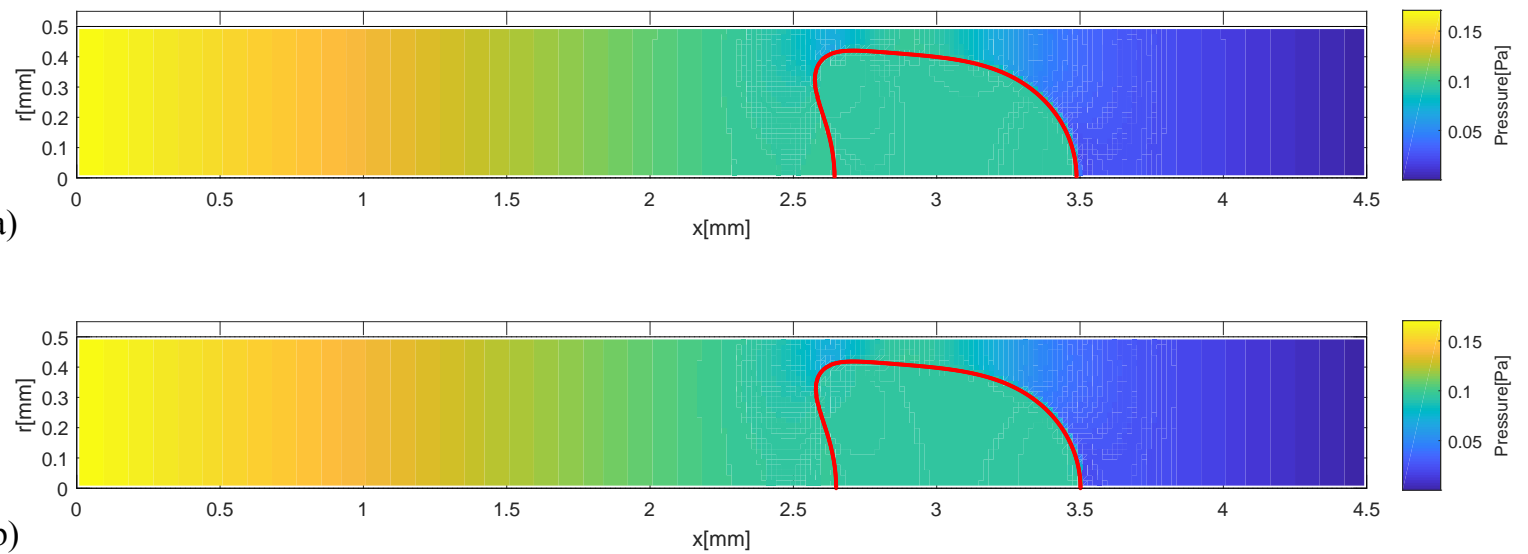

(c)

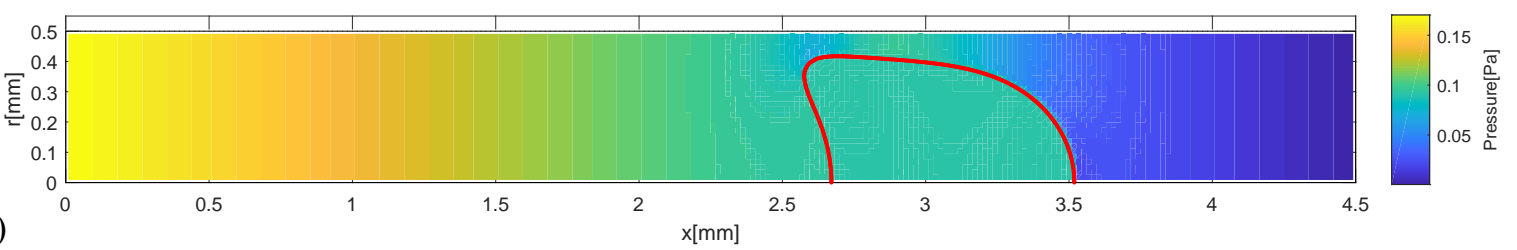

Figure 5.32: Pressure field for capsules flowing through the straight capillary: (a) Skalak's membrane material. (b) Hooke's law. (c) Neo-Hookean membrane material at $\bar{a}=0.9, \alpha=0.0, C a_{s}=0.05$, and $R e=1.0$. 


\section{4}

\section{Constricted capillary flow}

In the previous section, the formulation and numerical implementation of the axisymmetric flow solver were validated by comparing the membrane deformation in a straight capillary with results available in the literature.

This section is devoted to study capsule flow in an axisymmetric capillary with a constriction, to determine suspended capsule dynamics, and the consequent reduction of the flow mobility. Similarly to previous sections, for a given flow rate, the pressure difference is determined for different membrane properties, materials, and dimensions. The dimensionless parameters are the same listed previously.

The capillary geometry is fixed. The inlet and constriction radii are $R_{s}=0.5 \mathrm{~mm}$ and $R_{0}=0.215 \mathrm{~mm}$, respectively. The capillary length is $L=4.5 \mathrm{~mm}$. In general, a mesh of $29 \times 261$ quadrilateral elements produces mesh-independent results, as seen in Fig. 5.33-a. The membrane grid varies dynamically around 256 Lagrangian points, and a time step $\Delta t=10^{-4} \mathrm{~s}$ leads to time step independent results (see Fig. 5.33-b) in most cases.

The inner and outer liquid phases share the same density $\rho=1000 \mathrm{~kg} / \mathrm{m}^{3}$ and viscosity $\mu=0.001$ Pa.s from the previous sections, as well as the same properties of the membrane material, Poisson's ratio $\nu=0.499$ and a membrane thickness $h=0.01 \mathrm{~mm}$. The axisymmetric cases studied are summarized in Table 5.3 .

Table 5.3: Values of parameters for the constricted capillary flow.

\begin{tabular}{|l|l|}
\hline Parameters & Values \\
\hline $\bar{a}$ & {$[1.63,1.86]$} \\
\hline$\alpha$ & {$[0.025,0.05,0.1]$} \\
\hline$\beta$ & 0.43 \\
\hline$C a_{s}$ & {$[0.005,0.02,0.05$} \\
& $0.06,0.1,0.3,1.0]$ \\
\hline$R e$ & 1.0 \\
\hline Membrane material & SK, HK \\
\hline
\end{tabular}

At initial time, the liquid phase is at rest and the capsule has a spherical shape. The capsule is located at $x=0.45 \mathrm{~mm}$, as shown in Fig. 5.34. There is a pressure difference between inner and outer phases, associated with the 
(a)

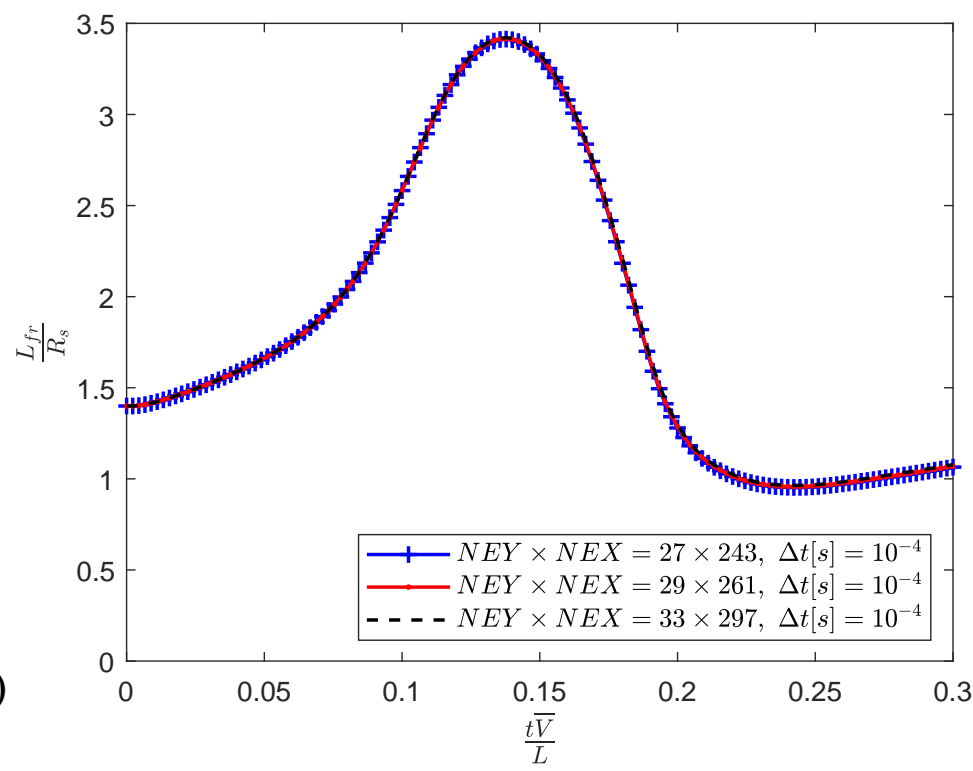

(b)

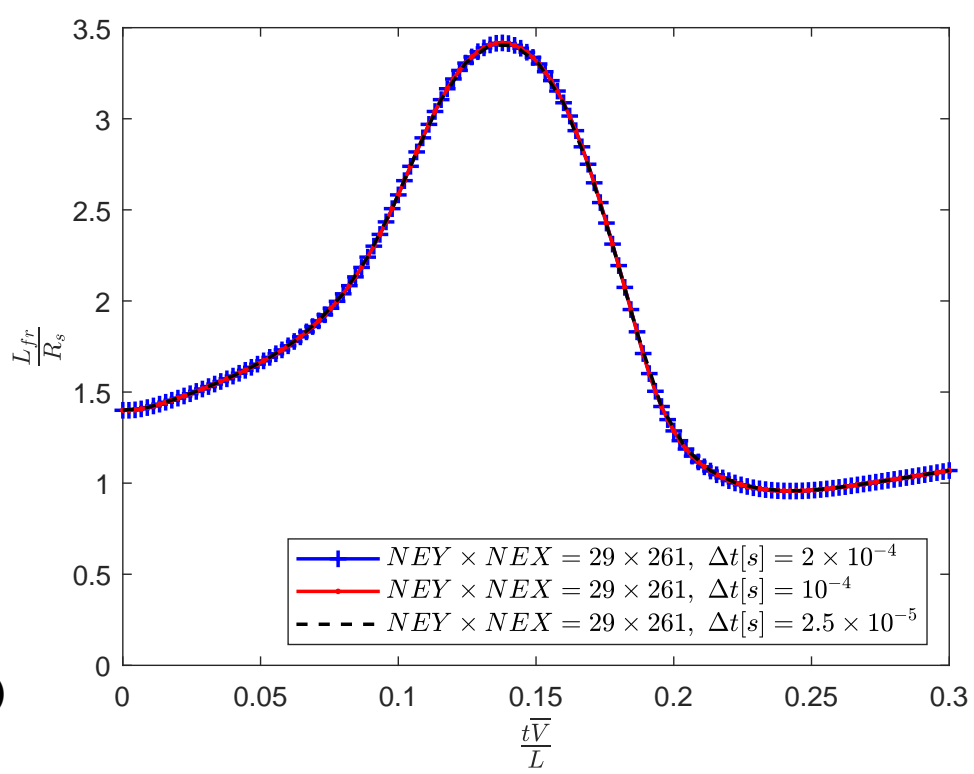

Figure 5.33: Temporal evolution of deformation $L_{f r}$ : (a) Different grids. (b) Different time steps at $\bar{a}=1.63, \alpha=0.1, C a_{s}=0.05$, and $R e=1.0$.

preinflation parameter $\alpha$, equal to $2 T_{0} h / a$.

Figures 5.35, 5.36, 5.37, and 5.38 illustrate the temporal evolution of the flow field and the capsule configuration (red line in the plot) as it flows through the constricted capillary at $\bar{a}=1.63, \alpha=0.1, R e=1.0$ for $C a_{s}=0.005$, $C a_{s}=0.02, C a_{s}=0.06$, and $C a_{s}=0.1$, respectively. As the capsule starts moving to narrower regions, the inlet pressure rises in order to pressurize 


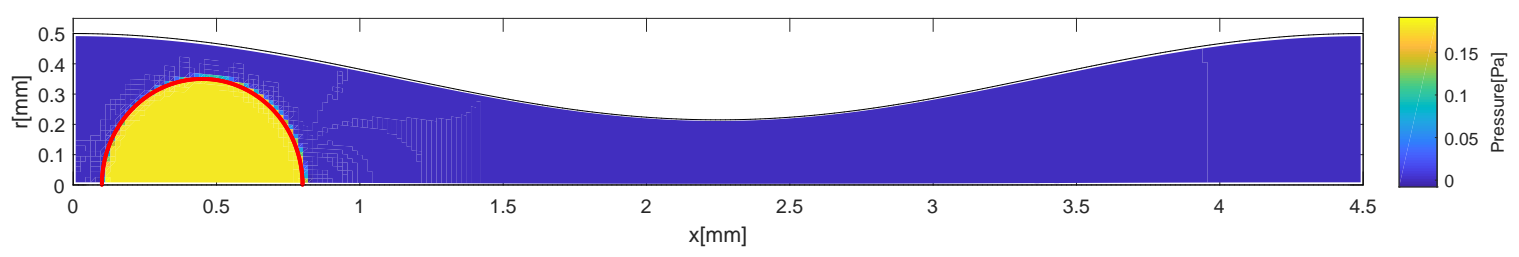

Figure 5.34: Initial pressure field and capsule configuration at $\bar{a}=1.63$, $\alpha=0.1, C a_{s}=0.02$, and $R e=1.0$.

(a)

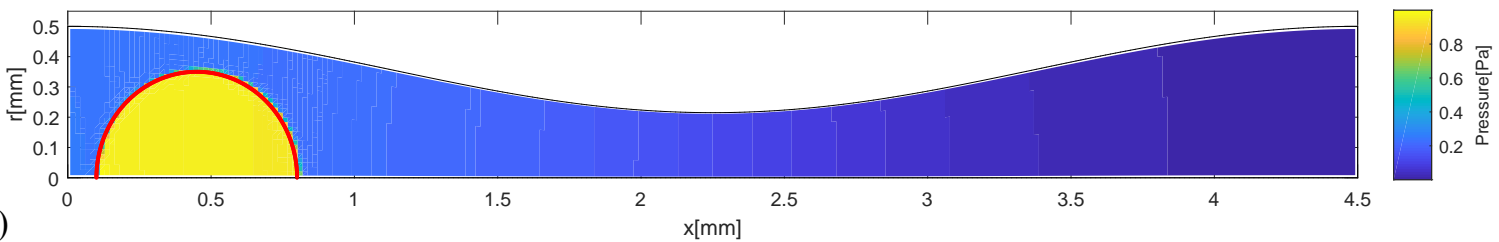

(b)
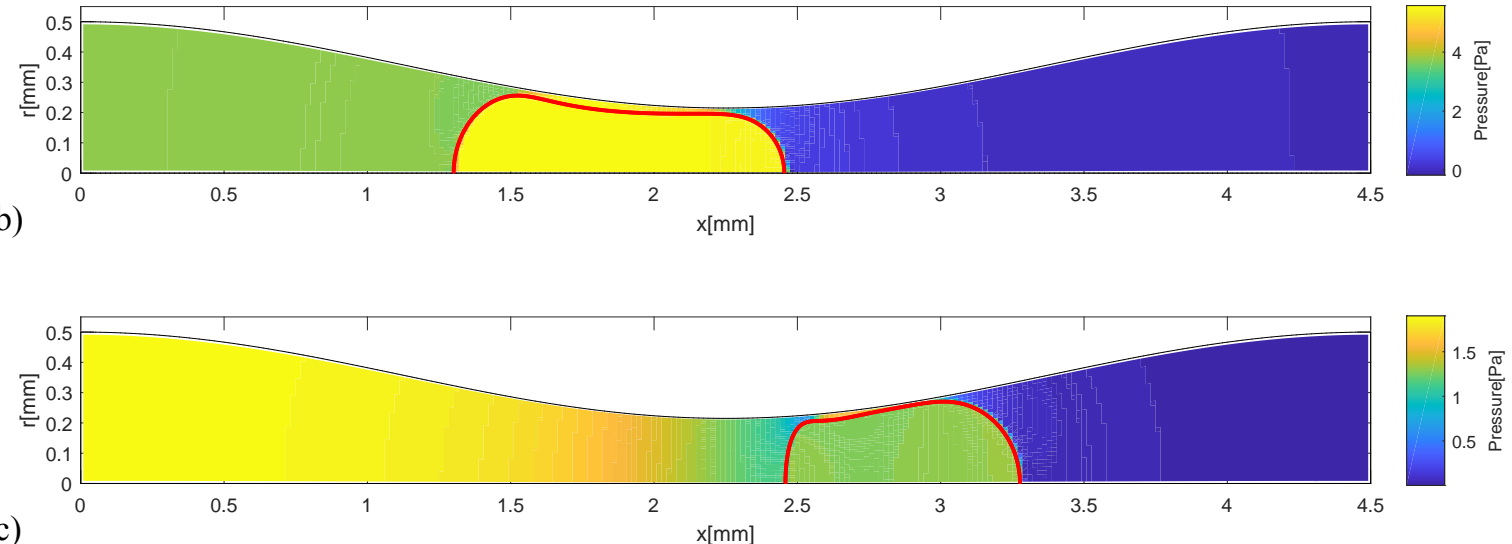

(d)

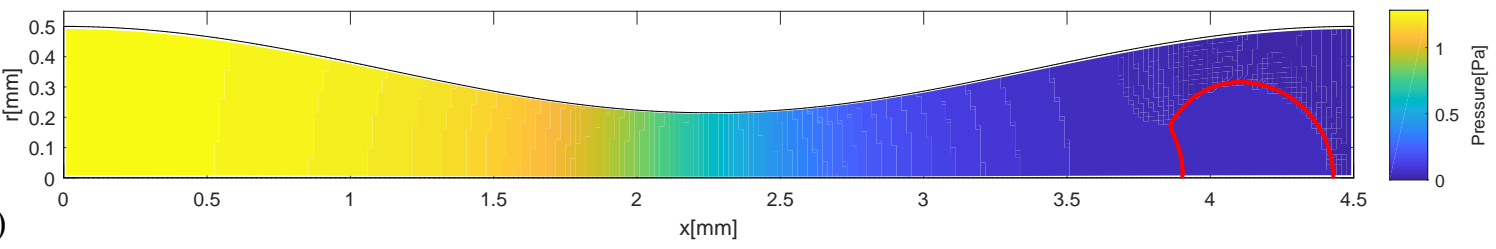

Figure 5.35: Temporal evolution of the membrane and pressure field: (a) $t=0.0001 s$; (b) $t=0.6013 \mathrm{~s}$; (c) $t=0.82 \mathrm{~s}$; and (d) $t=1.4 \mathrm{~s}$ at $\bar{a}=1.63$, $\alpha=0.1, C a_{s}=0.005$, and $R e=1.0$.

the system, and get the capsule through the constriction. At this point, the capsule front end is accelerated stretching the membrane. When leaving the constriction, the capsule starts to relax and the system depressurizes causing a decrease of the inlet pressure. The membrane reshape observed is because the capsule rear end moves faster than the front end, as pointed out by Rorai et al. [1]. Capsule flow at higher surface capillary number $C a_{s}$ tends to deform more at the symmetry line, where velocity is higher. Moreover, it maintains the croissant-like rear after passing the constriction. 
(a)

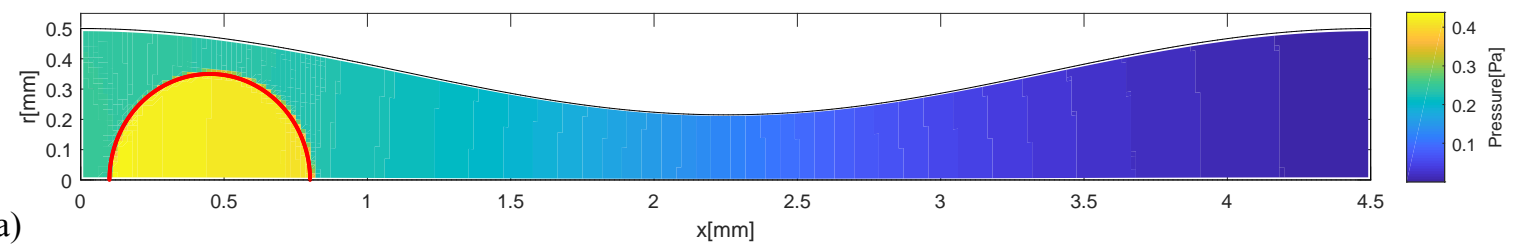

(b)

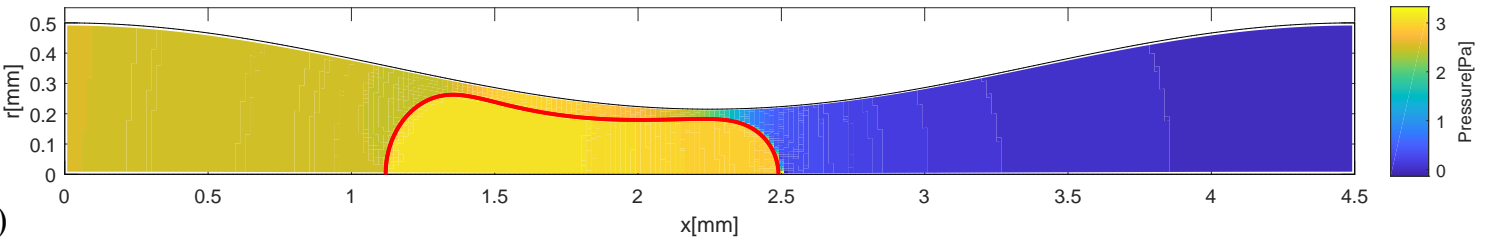

(c)
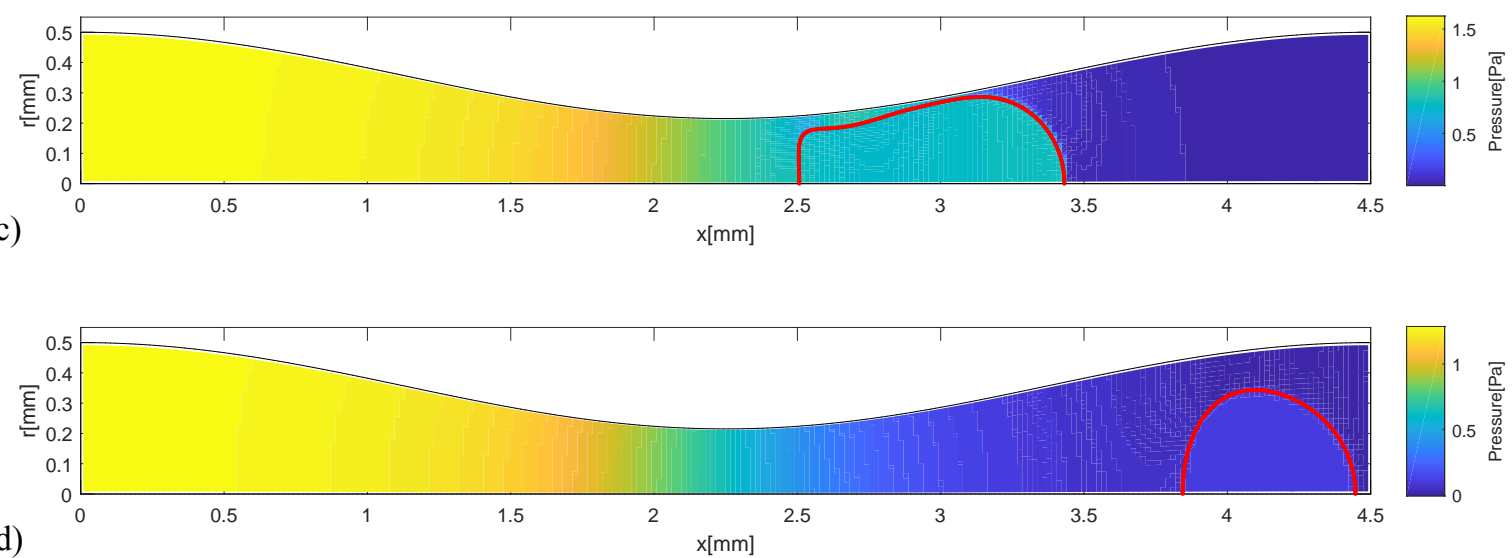

Figure 5.36: Temporal evolution of the membrane and pressure field: (a) $t=0.0001 s$; (b) $t=0.5546 \mathrm{~s}$; (c) $t=0.82 \mathrm{~s}$; and (d) $t=1.4 \mathrm{~s}$ at $\bar{a}=1.63$, $\alpha=0.1, C a_{s}=0.02$, and $R e=1.0$.

Figures 5.35-d, 5.36-d, 5.37-d, and 5.38-d show that there is loss of mass of the fluid contained inside the membrane after leaving the constriction. For the referred cases, the average mass loss is $-16 \%$, with the critical loss $-30 \%$ at the lowest $C a_{s}$ explored. Therefore, there is no doubt that this numerical issue gets worse in the axisymmetric flow than in the 2-D planar flow, because for a given flow condition, the former presents much higher velocities at the constriction, that may challenge the velocity interpolation. In fact, Peskin \& Printz [53] discussed the importance in having the appropriate interpolation scheme of velocity field at the membrane position $\mathbf{X}$, and hence they proposed a divergence operator in the context of finite difference method. In this work, this numerical issue shows the limits of the implementation, that might be attenuated not only by dynamically diminishing the time step and refining the mesh, but implementing a higher-order implicit time integration method.

In order to aid the visualization of the capsule configuration, different 
(a)

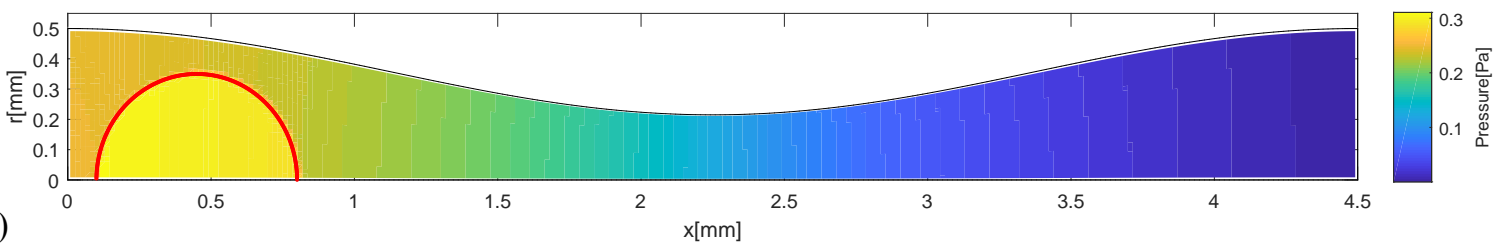

(b)

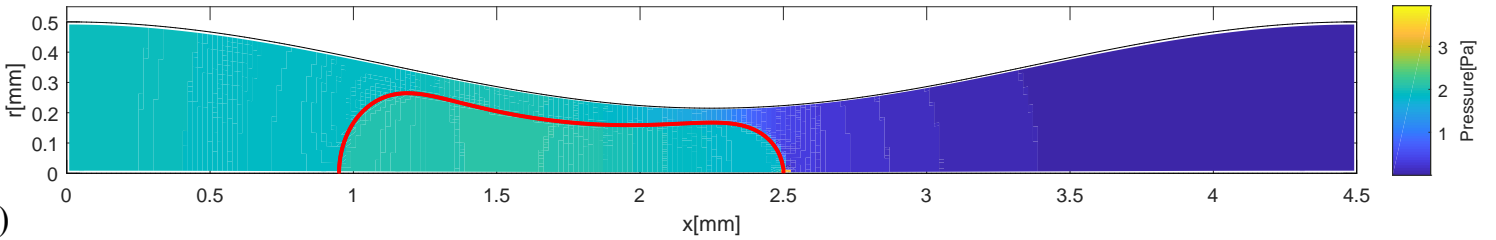

(c)
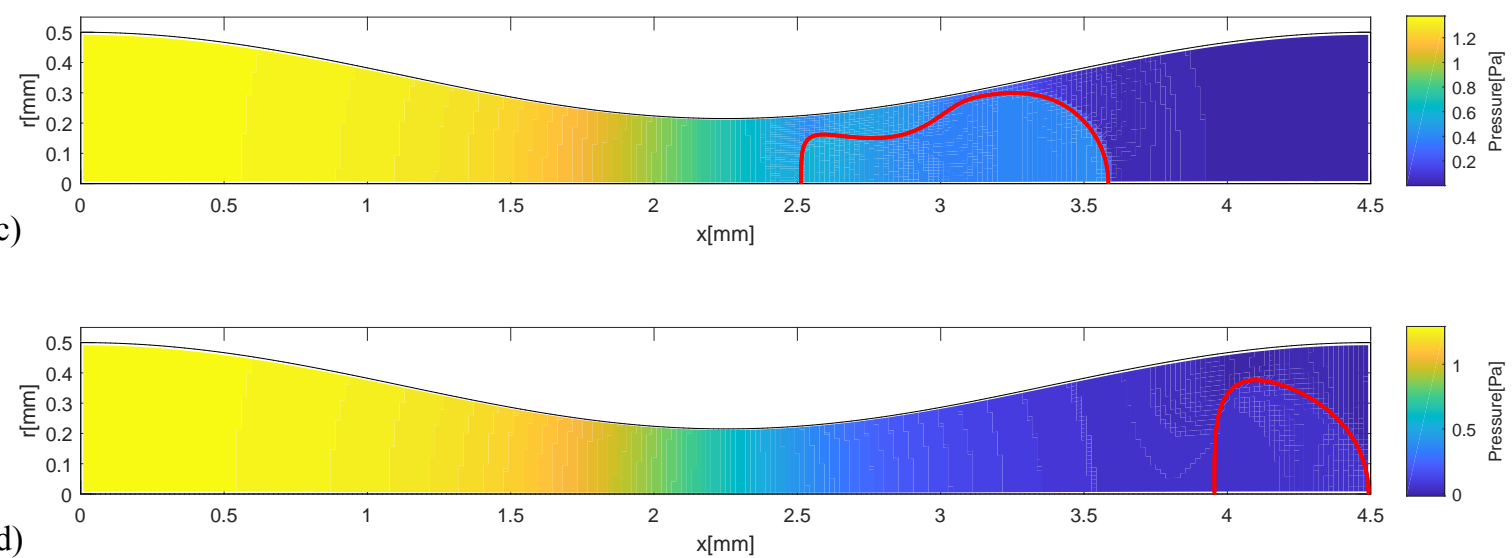

Figure 5.37: Temporal evolution of the membrane and pressure field: (a) $t=0.0001 s$; (b) $t=0.5004 s$; (c) $t=0.82 s$; and (d) $t=1.4 s$ at $\bar{a}=1.63$, $\alpha=0.1, C a_{s}=0.06$, and $R e=1.0$.

3 -D images generated from the axisymmetric solution at $C a_{s}=0.02$ are shown in Fig. 5.39. As previously reported by Do Nascimento et al. [8], three different deformation regimes are observed: predeformation, maximum deformation and recovery, as shown in Fig. 5.39-a, Fig. 5.39-b, and Fig. 5.39-d, respectively. The extra time, seen in Fig. 5.39-c, belongs to the recovery process. Note that the capsule is stretched along the $x$-axis when enters the narrowest cross-section, and compressed along the $x$-axis (accordingly, stretched along the $r$-direction) when leaving the constriction.

Figures 5.40-a and 5.40-b present the dimensionless meridional and azimuthal membrane stresses $T_{1}$ and $T_{2}$ at different times for the conditions of the flow presented in Fig. 5.13, i.e. $C a_{s}=0.06$ and $R e=1.0$. Initially, membrane stresses $T_{1}$ and $T_{2}$ are the same, equal to $T_{0}$ and uniformly distributed. As already mentioned, there are oscillations and a stress jump near the capsule tip $\left(s_{1}=0\right)$, which might be a numerical artifact related to membrane grid discretization, boundary condition, or even the time step used. As time 
(a)

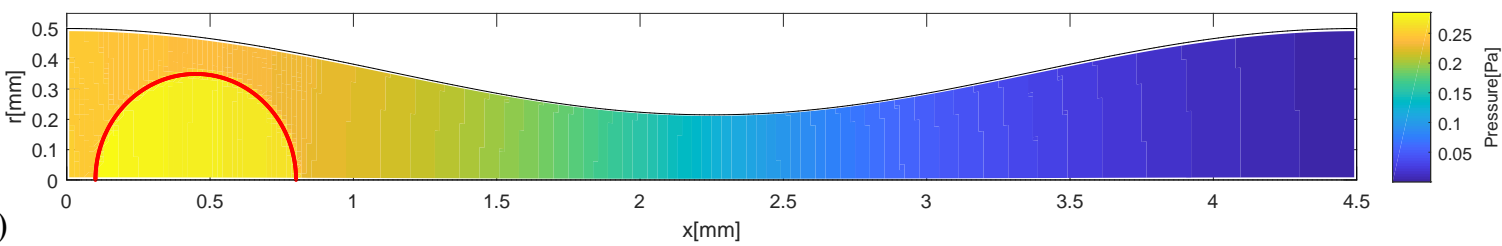

(b)

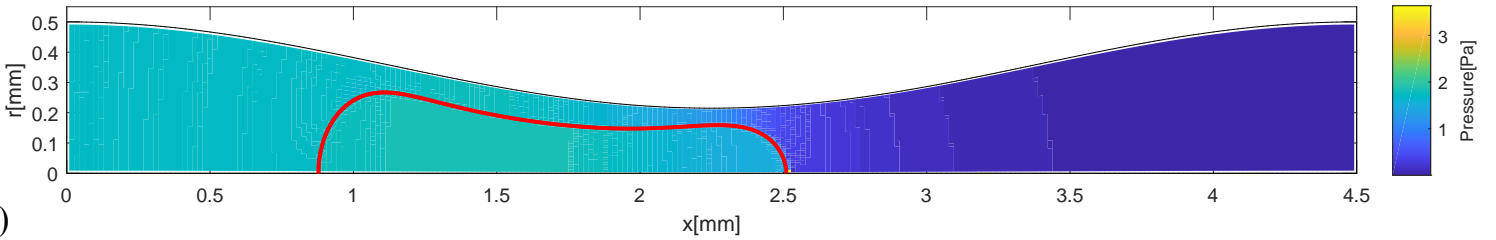

(c)
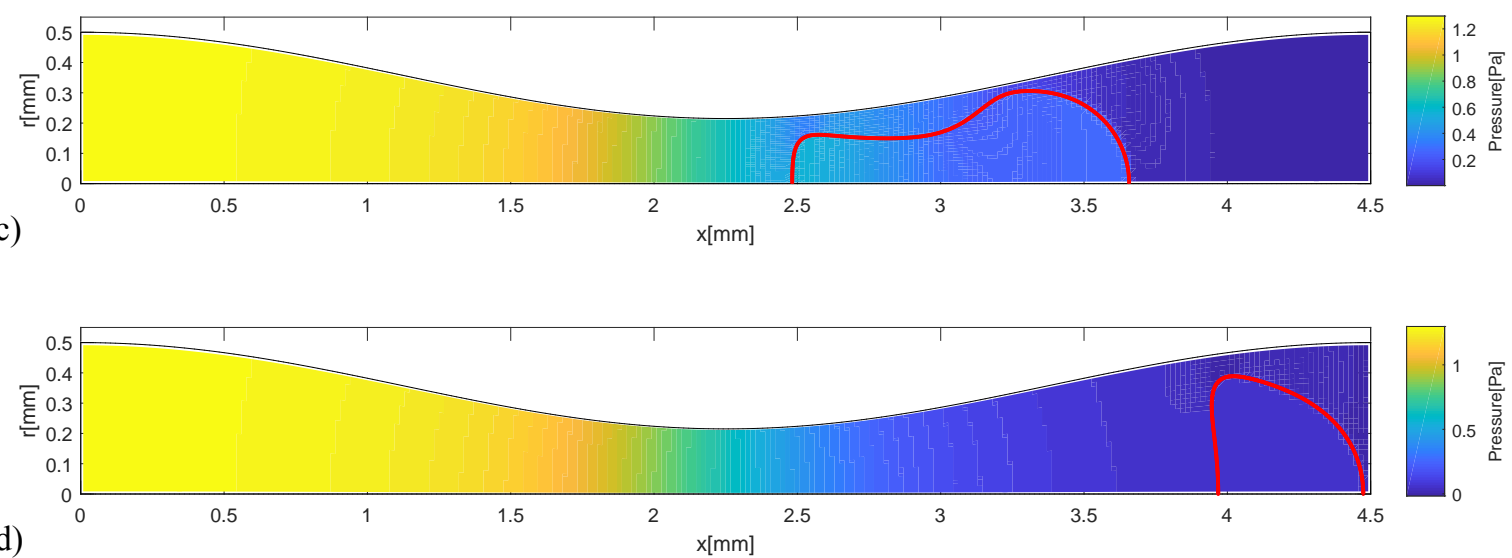

Figure 5.38: Temporal evolution of the membrane and pressure field: (a) $t=0.0001 \mathrm{~s}$; (b) $t=0.4747 \mathrm{~s}$; (c) $t=0.82 \mathrm{~s}$; and (d) $t=1.35 \mathrm{~s}$ at $\bar{a}=1.63$, $\alpha=0.1, C a_{s}=0.1$, and $R e=1.0$.

elapses, the capsule deforms and the membrane is elongated or compressed. Notice that, the front region of the capsule is under higher stresses than the rear region, suggesting the region where rupture may take place. After the constriction, the membrane relaxes and the stresses may fall so much that eventually the membrane is compressed leading to wrinkles formation (see Fig. 5.40-c), as discussed by Carroll [13].

Figure 5.41 shows the temporal evolution of the pressure difference between inlet and outlet for different values of surface capillary number $C a_{s}$. The pressure difference is presented in units of the steady-state pressure difference of the continuous phase flow $\Delta P_{c}$. The pressure difference for the single-phase flow is represented by the black continuous line. Similar to what was observed in the constricted channel flow, the inlet pressure rises in order to pass the capsule through the constriction, reaching the maximum pressure moments before the capsule center of gravity is at the constriction. As already observed, the pressure peak rises as the surface capillary number 


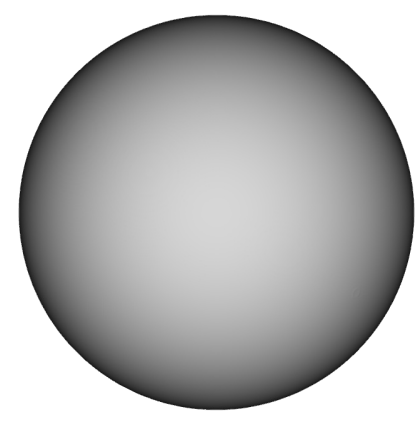

(a)

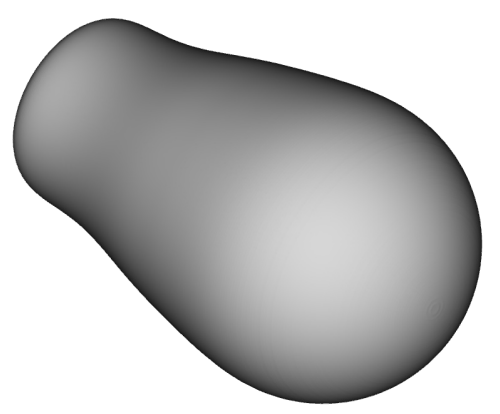

(c)

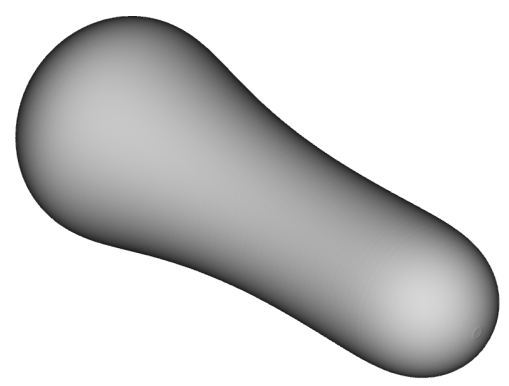

(b)

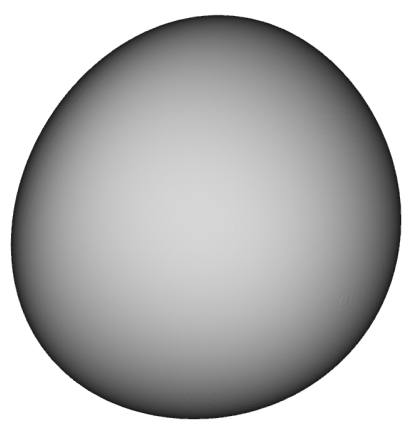

(d)

Figure 5.39: Temporal evolution of the capsule shape: (a) $t=0.0001 s$; (b) $t=0.5546 \mathrm{~s}$; (c) $t=0.82 \mathrm{~s}$; and (d) $t=1.4 \mathrm{~s}$ at $\bar{a}=1.63, \alpha=0.1, C a_{s}=0.02$, and $R e=1.0$.

falls. It is worth noting that after passing the constriction, there is a second pressure peak, which delays as $C a_{s}$ increases, that is associated with a rebound effect or stretching in the $r$-direction, as pointed out by Rorai et al. [1].

Similarly to previous sections, Fig. 5.42 depicts the minimum thickness of the lubricating liquid film rescaled by the inlet capillary radius $R_{s}$ as a function of surface capillary number $C a_{s}$, and described by the following power-law function:

$$
\frac{h_{\min }}{R_{s}} \approx C a_{s}^{0.60}
$$

The effect of the preinflation parameter $\alpha$ on the inlet pressure evolution is presented in Fig. 5.43 at $\bar{a}=1.63, C a_{s}=0.06$, and $R e=1.0$. Observe that when compared to the corresponding 2-D planar flow in Fig. 5.17, pressure response slightly varies with the preinflation parameter $\alpha$, showing that for the axisymmetric flow and Hooke's law membrane materials, the capsule flow is less sensitive to $\alpha$. 
(a)

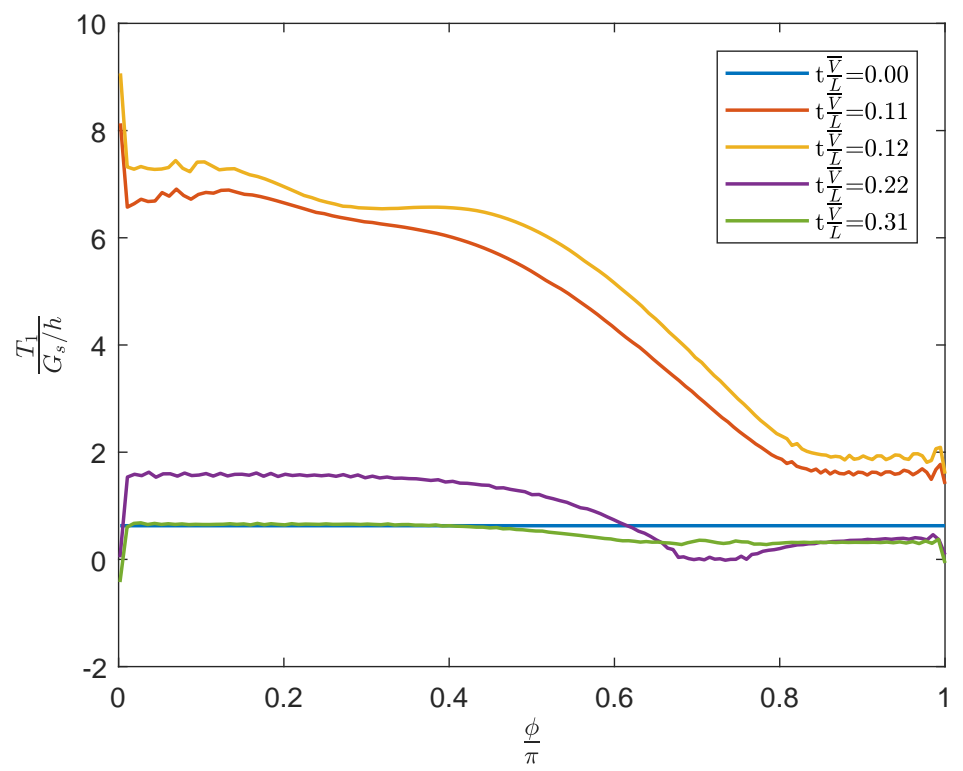

(b)

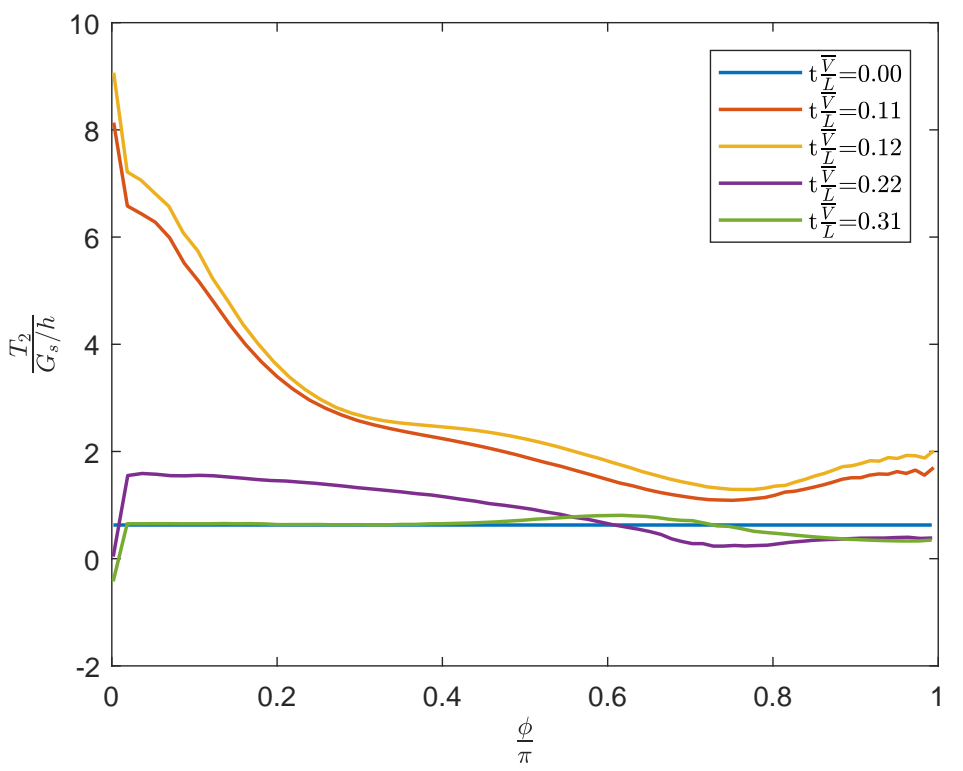

(c)

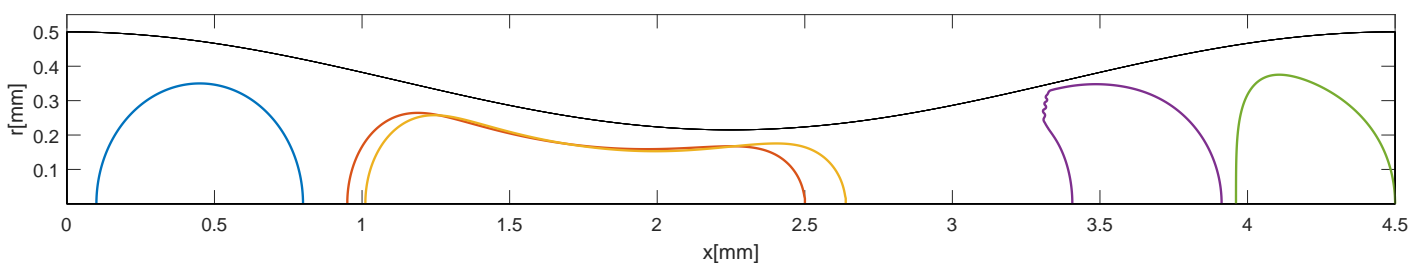

Figure 5.40: (a) Temporal evolution of membrane stress $T_{1}$ along angular position $\phi$. (b) Temporal evolution of membrane stress $T_{2}$ along angular position $\phi$. (c) Capsule position at $\bar{a}=1.63, \alpha=0.1, C a_{s}=0.06$, and $R e=1.0$. 


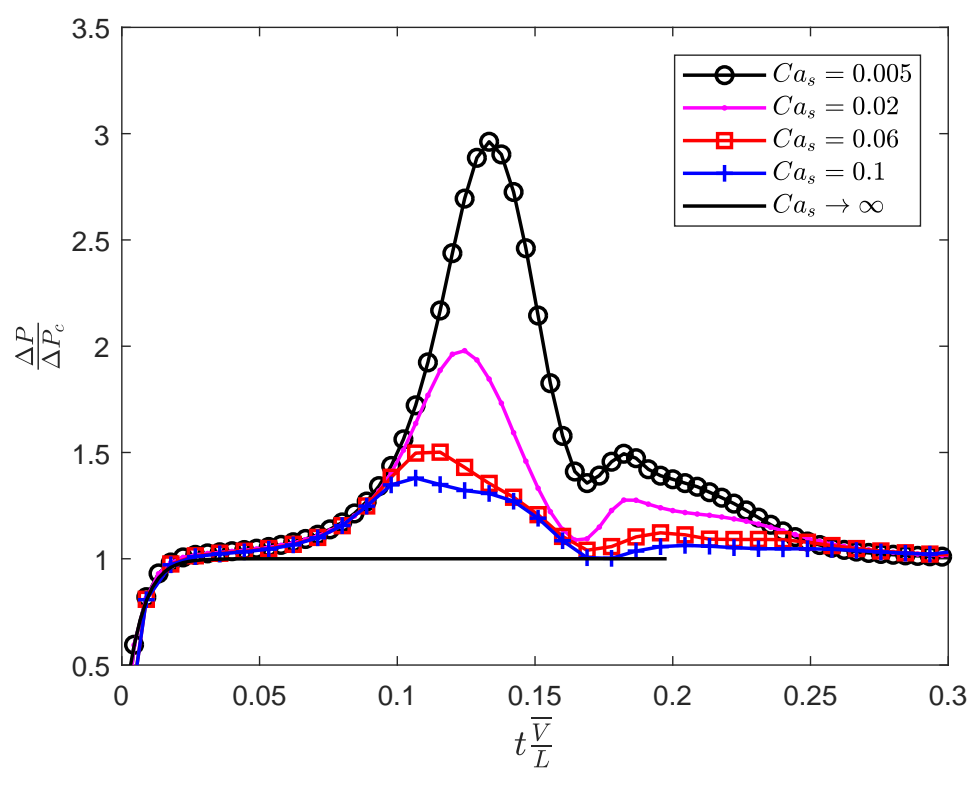

Figure 5.41: Temporal evolution of inlet pressure at $\bar{a}=1.63, \alpha=0.1$, and $R e=1.0$ for different values of surface capillary number $C a_{s}$.

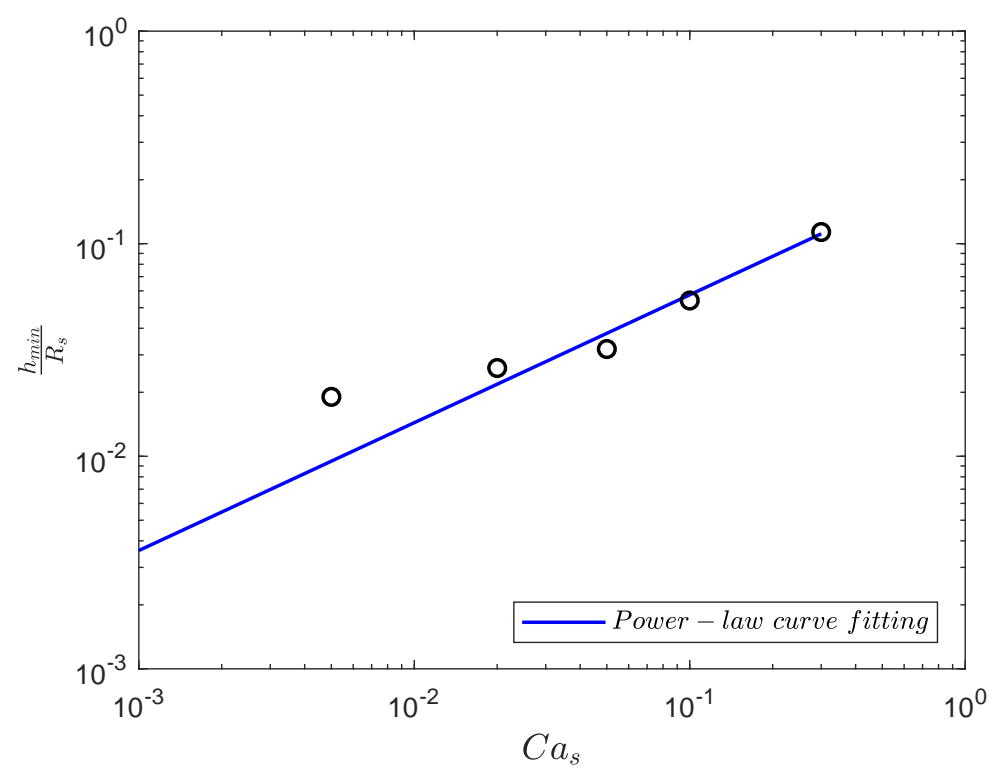

Figure 5.42: Minimum lubricating film thickness at $\bar{a}=1.63, \alpha=0.1$, and $R e=1.0$ as a function of surface capillary number $C a_{s}$.

Figure 5.44 illustrates the effect of the capsule radius $a$ on the inlet pressure evolution rescaled by the pressure difference of the single-phase flow. As already mentioned, larger capsules tend to decrease the liquid film thickness leading to higher inlet pressure in order to get the capsule through the throat. 


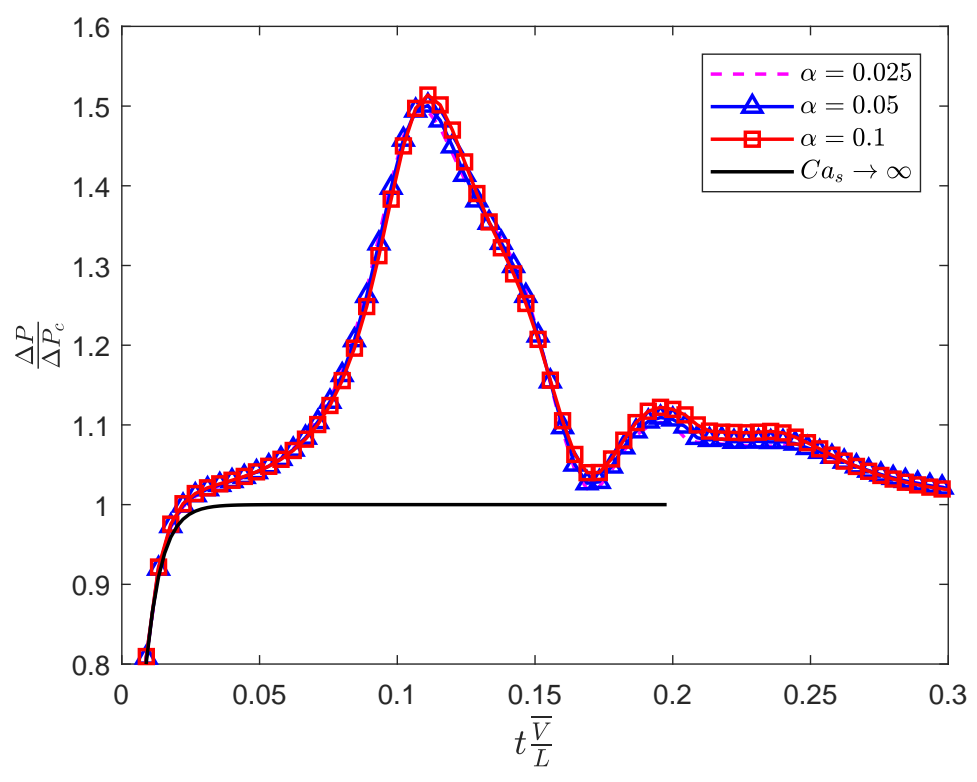

Figure 5.43: Temporal evolution of inlet pressure at $\bar{a}=1.63, C a_{s}=0.06$, and $R e=1.0$ for different values of preinflation parameter $\alpha$.

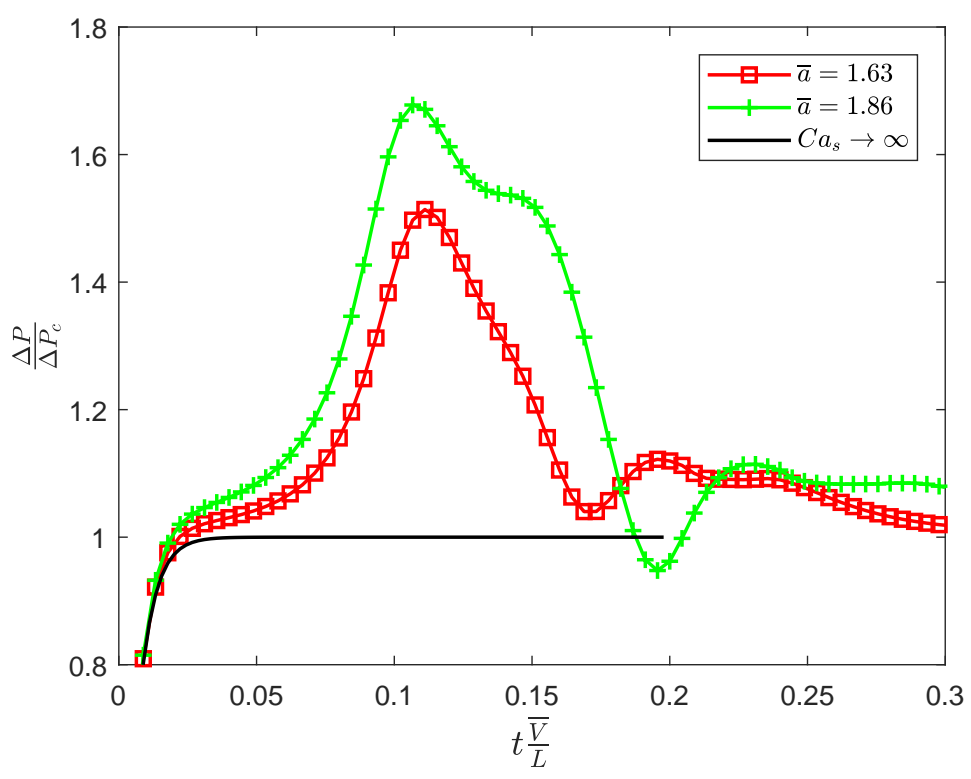

Figure 5.44: Temporal evolution of inlet pressure at $\alpha=0.1, C a_{s}=0.06$, and $R e=1.0$ considering different dimensionless capsule radii $\bar{a}$.

Figure 5.45 shows the capsule velocity $u_{C G}$ rescaled by the inlet mean velocity $\bar{V}$ as a function of the center of gravity of the capsule $X_{C G}$ for different cases. As expected, capsule velocity increases when it approaches the narrowest region, and decreases when the capsule exits the throat. By 
mass conservation, the case $C a_{s} \rightarrow \infty$ reaches its maximum velocity at the throat, i.e. $u_{0} / \bar{V}=\left(u_{s} / \bar{V}\right) /\left(R_{0} / R_{s}\right)^{2}=2 / \beta^{2}=10.82$. Observe that, for a membrane material described by Hooke's law, the peak of capsule velocity does not monotonically increase as $C a_{s}$ increases. In fact, there is a critical surface capillary number at which the maximum capsule velocity starts to decrease until it reaches its asymptotic value at $C a_{s} \rightarrow \infty$. In the limit, the softest capsule $\left(C a_{s} \rightarrow \infty\right)$ is purely advected by the flow.

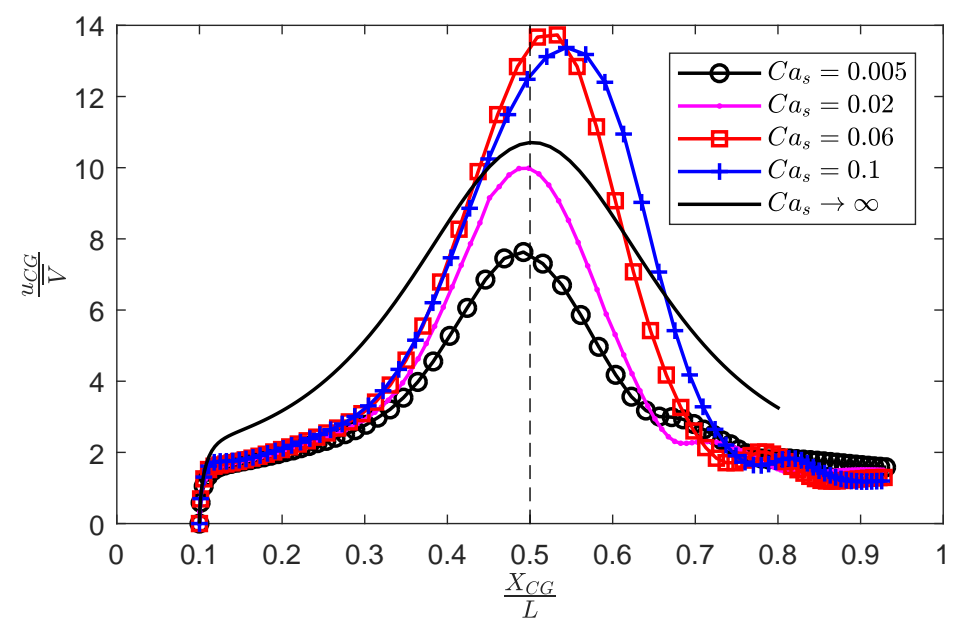

Figure 5.45: Capsule velocity along the $x$-axis for different values of surface capillary number $C a_{s}$. At $\bar{a}=1.63, \alpha=0.1$, and $R e=1.0$.

This change of behavior is clearly observed in Fig. 5.46, which also shows the response from a membrane described by Skalak's model. For the range of $C a_{s}$ explored, the maximum capsule velocity monotonically increases as the capsule softens, i.e. the softer the capsule, the faster is the capsule at the constriction, until reaching a maximum around $C a_{s}=0.1$ and starts to decline very slowly.

Similarly to the constricted channel flow section, Fig. 5.47 shows the mobility reduction factor $f$ for the suspended capsule flow through a constricted capillary using different membrane materials. As already discussed, higher peaks of pressure difference cause lower factor $f$, i.e. an important blocking effect due to the capsule. In contrast to the referred previous section, the mobility reduction factor $f$ is less sensitive to the preinflation parameter $\alpha$, and mainly depends on $C a_{s}$ and capsule radius $a$ at a given $R e$. Thus, stiffer capsules, i.e. lower $C a_{s}$, have a strong associated blocking mechanism (low $f$ ). Similar behavior is observed with larger capsules. 


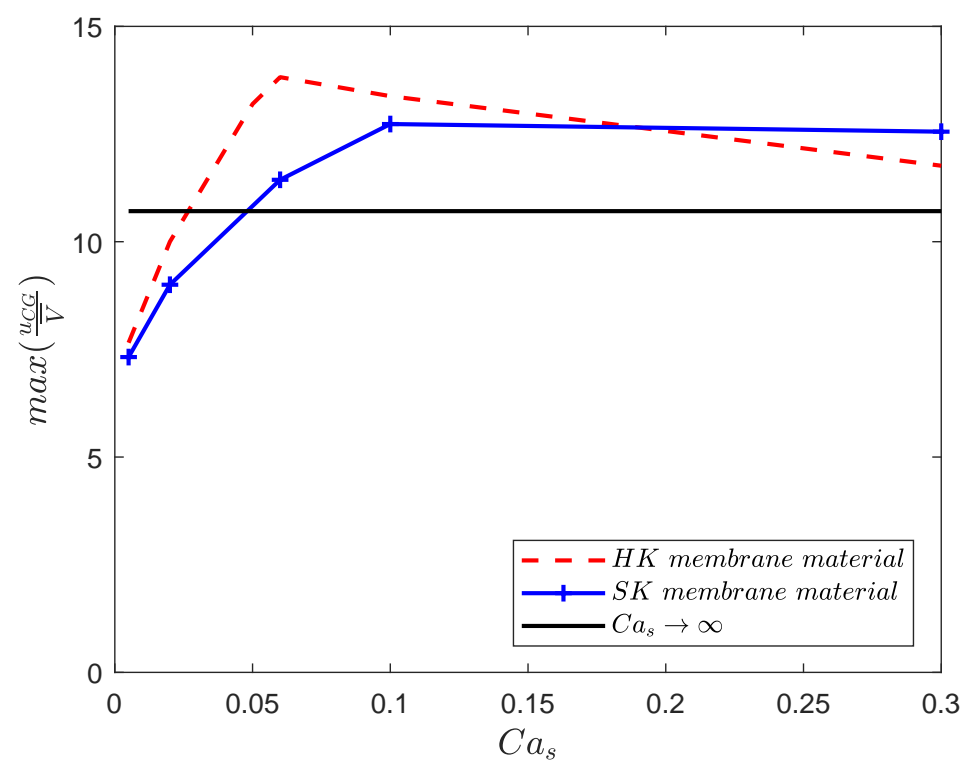

Figure 5.46: Maximum capsule velocity as a function of surface capillary number $C a_{s}$ for different membrane materials. At $\bar{a}=1.63, \alpha=0.1$, and $R e=1.0$.

As pointed out by Roca et al. [56], it is clear the effect of the low local surface capillary number on the water phase mobility reduction by diverting flow to alternative paths in porous media. Since the local capillary number decreases as the distance from the injector well increases, the dependence of the water phase mobility could be used to estimate the location inside a porous medium where mobility reduction occurs, by adjusting microcapsule elastic properties. The goal is to obtain low values of surface capillary number at the displacement front in order to effectively block pores.

Finally, regarding membrane materials, Fig. 5.47 also illustrates its effect on the mobility reduction factor $f$. Curves in green and magenta show the response of Skalak's model and Hooke's law materials, respectively. It is clear the strain-hardening behavior for Skalak's materials. In fact, for the range of $C a_{s}$ studied, stronger blocking mechanism of Skalak's materials starts at higher $C a_{s}$. 


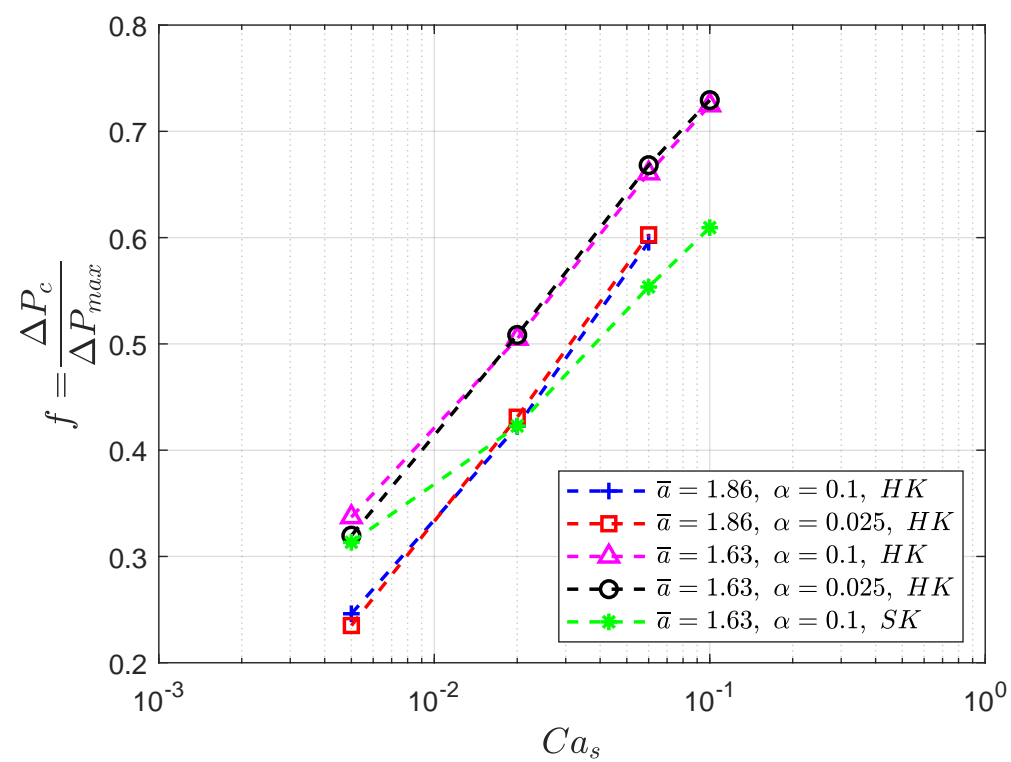

Figure 5.47: Mobility reduction factor $f$ as a function of $C a_{s}$ for different membrane materials, dimensionless capsule radii $\bar{a}$, and preinflation parameters $\alpha$ at $R e=1.0$. 


\section{6}

\section{Conclusions and Future work}

Flow of suspended capsules can be used to block preferential path of the water phase during oil displacement in porous media, and divert the water phase to pores with oil. The mobility change of the water phase is associated with the underlying physical phenomenon at the pore-level. In this work, a 2-D constricted channel and an axisymmetric capillary were used as models of a pore throat connecting two adjacent pore bodies. Furthermore, this study considered inertial effects (i.e. finite Reynolds numbers) and fully discussed pressure response as a function of different parameters because its important role in the capsule blocking mechanism.

Flow of a suspended capsule, modeled as a 1-D structure, through a 2-D constricted channel (or an axisymmetric constricted capillary) defines a fluid-structure interaction problem. The coupling between fluid and capsule membrane dynamics was taken into account by computing an extra source term in the Navier-Stokes equations, as well as advecting the membrane by the fluid velocity at each time step.

The set of transient nonlinear partial differential equations that describes fluid and capsule membrane dynamics was solved using the finite element method and the immersed boundary method.

Simple shear flow and straight capillary flow were used to validate formulation and numerical implementation for the 2-D planar flow and the axisymmetric flow, respectively. Regarding the simple shear flow, it was found that capsule deformation and orientation agree well with results available in the literature. For the straight capillary flow, on the other hand, results showed that the proposed model can recover the capsule shape and deformation, as well as membrane stresses when compared to other methodologies found in the literature.

Rise of the pressure difference needed to drive the flow at a given flow rate as the capsule flows through the constriction characterizes the mobility 
reduction of the flow. Results showed the influence of dimensionless capsule radius $\bar{a}$, preinflation parameter $\alpha$, and surface capillary number $C a_{s}$ for a given geometry and Reynolds number Re on mobility reduction factor $f$. A strong mobility reduction corresponds to low values of $f$. Thus, $f$ decreases as $C a_{s}$ decreases. For a given $C a_{s}$, larger capsules and higher prestress parameters lead to lower $f$.

Results of the present study can be used to design capsule properties such that pore blocking is strong at desired locations of the porous media leading to maximize oil displacement.

Regarding future work, some suggestions include the modeling of inner and outer fluids with different properties. In the current study, inner and outer fluids had the same properties. By including this new feature, it would be possible to explore the effect of different viscosity ratios on capsule flow, for instance.

Concerning boundary conditions, at the outlet plane, a fully developed flow was defined, i.e. $\mathbf{n} \cdot \boldsymbol{\nabla u}=\mathbf{0}$. This boundary condition could be relaxed so that the flow develops freely. In principle, including the necessary extra terms in the implemented natural conditions at the outlet plane would be a straightforward procedure.

Modeling of the force in the axisymmetric geometry considers that a 3-D membrane element projects onto its tangent plane as a rectangular region. This clearly is an approximation. A recommendation is made to approximate such membrane element as a trapezoidal region, this would lead to a better accounting of additional force components.

Furthermore, for some cases, membrane stresses along the arc-length presented oscillations, especially higher at both capsule ends. In order to attenuate these oscillations, some suggestions are made, including cubic splines to interconnect membrane nodes and smoothing schemes, reducing time step because it was observed its direct effect on oscillations, and improvement of boundary conditions at capsule front and back ends, e.g. a higher-order derivative of $\mathbf{X}$ may be required.

Another way to extend this work is to include bending effects, which would require to compute higher-order derivatives of $\mathbf{X}$ with respect of the 
arc-length. For that reason, it would be interesting to use a cubic spline, as suggested previously.

Regarding results, there is certainly room to explore an even wider range of parameters. For instance, further study of inertial effects on capsule flow, i.e. run cases with higher values of Reynolds number Re. Moreover, extend obtained results for the Skalak's membrane material to different preinflation and capsule radius conditions. For Neo-Hookean membrane material, on the other hand, some initial results in the straight capillary were presented showing its shear-softening behavior. Nonetheless, it was not possible to further explore this material because the implemented code was not able to handle large membrane deformations, especially at the front end of the capsule. Therefore, it is recommended to improve code capabilities by implementing a new remeshing scheme to address such large deformations.

An alternative way to evaluate the constriction effect would be to start simulations considering the capsule already deformed, i.e. the steady-state solution.

In the context of the immersed boundary method, mass loss is a wellknown problem. This study is not the exception. For the constricted channel flow, the average mass loss for different cases was acceptable and below $-6.5 \%$, with the worst performance $-13 \%$ at the lowest $C a_{s}=0.005$. For the constricted capillary flow, on the other hand, the average mass loss for different cases was below $-16 \%$, with the critical performance $-30 \%$ at the lowest $C a_{s}=0.005$. Thus, this numerical artifact gets worse as $C a_{s}$ decreases, indicating the limits of the present study. Some measures are suggested to attenuate this issue, including mesh refinement, with special emphasis on the constriction (to carry out this, the code will need to handle non-uniform meshes), using a second-order implicit time integration method and reducing time step. 


\section{Bibliography}

[1] RORAI, C.; TOUCHARD, A.; ZHU, L. ; BRANDT, L.. Motion of an elastic capsule in a constricted microchannel. Eur. Phys. J. E Soft Matter, 38:49, 2015.

[2] GUIDO, S.; TOMAIUOLO, G.. Microconfined flow behavior of red blood cells in vitro. C. R. Phys., 10(8):751-763, 2009.

[3] MCWHIRTER, J. L.; NOGUCHI, H. ; GOMPPER, G.. Flow-induced clustering and alignment of vesicles and red blood cells in microcapillaries. Proc. Natl. Acad. Sci. USA, 106(15):6039-6043, 2009.

[4] DIDAR, T. F.; TABRIZIAN, M.. Adhesion based detection, sorting and enrichment of cells in microfluidic lab-on-chip devices. Lab Chip, 10(22):3043-3053, 2010.

[5] MUZYKANTOV, V.. Drug delivery by red blood cells: Vascular carriers designed by Mother Nature. Expert Opin. Drug Deliv., 7(4):403-427, 2010.

[6] AMSTAD, E.. Capsules: Their past and opportunities for their future. ACS Macro Lett., 6:841-847, 2017.

[7] DATTA, S. S.; ABBASPOURRAD, A.; AMSTAD, E.; FAN, J.; KIM, S. H.; ROMONOWSKY, M.; SHUM, H. C.; SUN, B.; UTADA, A. S.; WINDBERGS, M.; ZHOU, S. ; WEITZ, D. A.. 25th anniversary article: Double emulsion templated solid microcapsules: Mechanics and controlled release. Adv. Mater., 26:2205-2218, 2014.

[8] DO NASCIMENTO, D.; AVENDAÑO, J.; MEHL, A.; MOURA, M.; CARVALHO, M. ; DUNCANSON, W.. Flow of tunable elastic microcapsules through constrictions. Sci. Rep., 7:11898, 2017.

[9] RIBEIRO, R. C. S.. 3-D visualization of oil displacement by a suspension of microcapsules. Master's thesis, Pontifícia Universidade Católica do Rio de Janeiro, 2019. 
[10] ØREN, P.-E.; BAKKE, S.. Reconstruction of Berea sandstone and pore-scale modelling of wettability effects. J. Pet. Sci. Eng., 39(3):177-199, 2003.

[11] BARTHÈS-BIESEL, D.. Motion and deformation of elastic capsules and vesicles in flow. Annu. Rev. Fluid Mech., 48(1):25-52, 2016.

[12] PESKIN, C.. Flow patterns around heart valves: A numerical method. J. Comput. Phys., 10(2):252-271, 1972.

[13] CARROLL, R.. Computational study of droplet and capsule flow in channels with inertial effects. PhD thesis, University of New Hampshire, 2014.

[14] MOKKEN, F. C.; KEDARIA, M.; HENRY, C. P.; HARDEMAN, M. R. ; GELB, A. W.. The clinical importance of erythrocyte deformability, a hemorrheological parameter. Ann. Hematol., 64:113-122, 1992.

[15] FREUND, J.. Numerical simulation of flowing blood cells. Annu. Rev. Fluid Mech., 46(1):67-95, 2014.

[16] BURGAIN, J.; GAIANI, C.; LINDER, M. ; SCHER, J.. Encapsulation of probiotic living cells: From laboratory scale to industrial applications. J. Food Eng., 104(4):467-483, 2011.

[17] BAKRY, A. M.; ABBAS, S.; ALI, B.; MAJEED, H.; ABOUELWAFA, M. Y.; MOUSA, A. ; LIANG, L.. Microencapsulation of oils: A comprehensive review of benefits, techniques, and applications. Compr. Rev. Food Sci. Food Saf., 15(1):143-182, 2016.

[18] WETZEL, B.; SCHAEFER, H.. Scanning electron microscope image of blood cells. National Cancer Institute. https://visualsonline. cancer.gov/details . cfm?imageid=2129 (accessed: 12.07.2020).

[19] BURNS, J. M.; YANG, X.; FOROUZAN, O.; SOSA, J. M. ; SHEVKOPLYAS, S. S.. Artificial microvascular network: A new tool for measuring rheologic properties of stored red blood cells. Transfusion, 52(5):1010-1023, 2012.

[20] FISCHER, T. M.; STÖHR-LIESEN, M. ; SCHIMID-SCHÖNBEIN, H.. The red cell as a fluid droplet: Tank tread-like motion of the human erythrocyte membrane in shear flow. Science, 202(4370):894-896, 1978. 
[21] ZHOU, H.; POZRIKIDIS, C.. Deformation of liquid capsules with incompressible interfaces in simple shear flow. J. Fluid Mech., 23:175-200, 1995.

[22] BREYIANNIS, G.; POZRIKIDIS, C.. Simple shear flow of suspensions of elastic capsules. Theoret. Comput. Fluid Dynamics, 13:327-347, 2000.

[23] POZRIKIDIS, C.. Effect of membrane bending stiffness on the deformation of capsules in simple shear flow. J. Fluid Mech., 440:269-291, 2001.

[24] SUI, Y.; CHEW, Y. T. ; LOW, H. T.. A lattice Boltzmann study on the large deformation of red blood cells in shear flow. Int. J. Mod. Phys. C, 18(6):993-1011, 2007.

[25] SUI, Y.; CHEW, Y. T.; ; ROY, P. ; LOW, H. T.. Inertia effect on the transient deformation of elastic capsules in simple shear flow. Comput. Fluids, 38(1):49-59, 2009.

[26] RACHIK, M.; BARTHÈS-BIESEL, D.; CARIN, M. ; EDWARDS-LÉVY, F.. Identification of the elastic properties of an artificial capsule membrane with the compression test: Effect of thickness. J. Colloid Interface Sci., 301(1):217-226, 2006.

[27] LEFEBVRE, Y.; LECLERC, E.; BARTHÈS-BIESEL, D.; WALTER, J. ; EDWARDS-LÉVY, F.. Flow of artificial microcapsules in microfluidic channels: A method for determining the elastic properties of the membrane. Phys. Fluids, 20(12):123102, 2008.

[28] RISSO, F.; COLLÉ-PAILLOT, F. ; ZAGZOULE, M.. Experimental investigation of a bioartificial capsule flowing in a narrow tube. J. Fluid Mech., 547:149-173, 2006.

[29] GUPSPUN, J.; DE LOUBENS, C.; TROZZO, R.; DESCHAMPS, J.; GEORGELIN, M.; EDWARDS-LÉVY, F. ; LEONETTI, M.. Perturbations of the flow induced by a microcapsule in a capillary tube. Fluid Dyn. Res., 49(3):035501, 2017.

[30] LECLERC, E.; KINOSHITA, H.; FUJII, T. ; BARTHĖS-BIESEL, D.. Transient flow of microcapsules through convergent-divergent microchannels. Microfluid. Nanofluid., 12:761-770, 2012.

[31] HU, X.-Q.; SALSAC, A.-V. ; BARTHÈS-BIESEL, D.. Flow of a spherical capsule in a pore with circular or square cross-setion. J. Fluid Mech., 705:176-194, 2012. 
[32] LEYRAT-MAURIN, A.; BARTHÈS-BIESEL, D.. Motion of a deformable capsule through a hyperbolic constriction. J. Fluid Mech., 279:135163, 1994.

[33] DIMITRAKOPOUlOS, P.; KURIAKOSE, S.. Determining a membrane's shear modulus, independent of its area-dilation modulus, via capsule flow in a converging micro-capillary. Soft Matter, 11(14):2782-2793, 2015.

[34] KUMAR, A.; GRAHAM, M.. Accelerated boundary integral method for multiphase flow in non-periodic geometries. J. Comput. Phys., 231(20):6682-6713, 2012.

[35] HOU, G.; WANG, J. ; LAYTON, A.. Numerical methods for fluidstructure - A review. Commun. Comput. Phys., 12(2):337-377, 2012.

[36] BOFFI, D.; GASTALDI, L.. The immersed boundary method: A finite element approach. In: COMPUTATIONAL FLUID AND SOLID MECHANICS 2003, p. 1263-1266. Elsevier Inc., 2003.

[37] ILINCA, F.; HÉTU, J.-F.. A finite element immersed boundary method for fluid flow around rigid objects. Int. J. Numer. Meth. Fluids, 65(7):856-875, 2011.

[38] LAI, M.-C.; PESKIN, C.. An immersed boundary method with formal second-order accuracy and reduced numerical viscosity. J. Comput. Phys., 160:705-719, 2000.

[39] SOTIROPOULOS, F.; YANG, X.. Immersed boundary methods for simulating fluid-structure interaction. Prog. Aerosp. Sci., 65:1-21, 2014.

[40] MITTAL, R.; IACCARINO, G.. Immersed boundary methods. Annu. Rev. Fluid Mech., 37(1):239-261, 2005.

[41] FLÜGGE, W.. Stresses in shells. Springer-Verlag Berlin Heidelberg, Berlin, 1st edition, 1960.

[42] PESKIN, C.; MCQUEEN, D.. A three-dimensional computational method for blood blow in heart I. Immersed elastic fibers in a viscous incompressible fluid. J. Comput. Phys., 81(2):372-405, 1989.

[43] EVANS, E.; SKALAK, R.. Mechanics and thermodynamics of biomembranes. Taylor \& Francis Group, Boca Raton, 1st edition, 1980. 
[44] LEFEBVRE, Y.; BARTHÈS-BIESEL, D.. Motion of a capsule in a cylindrical tube: Effect of membrane pre-stress. J. Fluid Mech., 589:157-181, 2007.

[45] LAC, E.; BARTHĖS-BIESEL, D.. Deformation of a capsule in simple shear flow: Effect of membrane prestress. Phys. Fluids, 17(7), 2005.

[46] SKALAK, R.; TOZEREN, A.; ZARDA, R. P. ; CHIEN, S.. Strain energy function of red blood cell membranes. Biophys. J., 13(3):245-264, 1973.

[47] HU, X.-Q.. Motion and deformation of capsules flowing in microfluidic channels. PhD thesis, Université de Technologie de Compiègne, 2013.

[48] CARVALHO, M.; VALÉRIO, J.. Introdução ao método de elementos finitos: Aplicação em dinâmica dos fluidos. SBMAC, 2012.

[49] ROCA, J. F.; CARVALHO, M. S.. Flow of a drop through a constricted microcapillary. Comput. Fluids, 87:50-56, 2013.

[50] MATLAB. version R2018a Update 6 (9.4.0.949201). The MathWorks Inc., Natick, 2018.

[51] HU, W.-F.; KIM, Y. ; LAI, M.-C.. An immersed boundary method for simulating the dynamics of three-dimensional axisymmetric vesicles in Navier-Stokes flows. J. Comput. Phys., 257:670-686, 2014.

[52] DUPONT, C.; TALLEC, P. L.; BARTHÈS-BIESEL, D.; VIDRASCU, M. ; SALSAC, A.-V.. Dynamics of a spherical capsule in a planar hyperbolic flow: Influence of bending resistance. Procedia IUTAM, 16:70-79, 2015.

[53] PESKIN, C.; PRINTZ, B.. Improved volume conservation in the computation of flows with immersed elastic boundaries. J. Comput. Phys., 105(1):33-46, 1993.

[54] LeOPÉRCIO, B. C.; MICHELON, M. ; CARVAlHO, M. S.. Deformation and rupture of microcapsules flowing through constricted capillary. 2021. Manuscript submitted for publication.

[55] COBOS, S.; CARVAlHO, M. S. ; ALVARADO, V.. Flow of oil-water emulsions through a constricted capillary. Int. J. Multiph. Flow, 35(6):507-515, 2009. 
[56] ROCA, J. F.; MENEZES, I. F. ; CARVALHO, M. S.. Mobility reduction in the flow of an elastic microcapsule through a constricted channel. Ind. Eng. Chem. Res., 60(5):2278-2289, 2021.

[57] BEER, F.; JOHNSTON, E.; DEWOLF, J. ; MAZUREK, D.. Mechanics of materials. Mc Graw Hill, New York, 6th edition, 2012. 
A

\section{Appendix}

\section{A.1}

\section{Determination of $T_{1}$ for the 2-D planar flow}

According to Fig. (3.2), the generalized Hooke's law for the multiaxial loading of a homogeneous isotropic material [57] can be written as

$$
\begin{aligned}
\varepsilon_{1} & =+\frac{T_{1}}{E}-\frac{\nu T_{2}}{E}-\frac{\nu T_{3}}{E}, \\
\varepsilon_{2} & =-\frac{\nu T_{1}}{E}+\frac{T_{2}}{E}-\frac{\nu T_{3}}{E}, \\
\varepsilon_{3} & =-\frac{\nu T_{1}}{E}-\frac{\nu T_{2}}{E}+\frac{T_{3}}{E} .
\end{aligned}
$$

Considering that the stress normal to the capsule surface $T_{3}$ is small when compared to other stresses $T_{1}$ and $T_{2}$, the following expressions are obtained:

$$
\begin{aligned}
& \varepsilon_{1}=+\frac{T_{1}}{E}-\frac{\nu T_{2}}{E}, \\
& \varepsilon_{2}=-\frac{\nu T_{1}}{E}+\frac{T_{2}}{E}, \\
& \varepsilon_{3}=-\frac{\nu T_{1}}{E}-\frac{\nu T_{2}}{E} .
\end{aligned}
$$

Additionally, there is no strain in the direction perpendicular to the $x y$ plane, $\varepsilon_{2}=0$, because the membrane is infinitely long in the $z$-direction. Thus, $T_{2}=\nu T_{1}$.

Substituting $T_{2}=\nu T_{1}$ into the first expression of eqs. (A-2), $T_{1}$ is finally obtained as $T_{1}=\left(\frac{E}{1-\nu^{2}}\right) \varepsilon_{1}$. 